Florida International University FIU Digital Commons

$11-5-2010$

\title{
A Highly Efficient Biometrics Approach for Unconstrained Iris Segmentation and Recognition
}

Yu Chen

Florida International University, ychen001@fiu.edu

DOI: $10.25148 /$ etd.FI10112006

Follow this and additional works at: https://digitalcommons.fiu.edu/etd

Part of the Electrical and Electronics Commons, and the Signal Processing Commons

\section{Recommended Citation}

Chen, Yu, "A Highly Efficient Biometrics Approach for Unconstrained Iris Segmentation and Recognition" (2010). FIU Electronic Theses and Dissertations. 310.

https://digitalcommons.fiu.edu/etd/310 


\title{
FLORIDA INTERNATIONAL UNIVERSITY
}

Miami, Florida

\section{A HIGH EFFICIENT BIOMETRICS APPROACH FOR UNCONSTRAINED IRIS SEGMENTATION AND RECOGNITION}

\author{
A dissertation submitted in partial fulfillment of the \\ requirements for the degree of \\ DOCTOR OF PHILOSOPHY \\ in \\ ELECTRICAL ENGINEERING \\ by \\ Yu Chen
}

2010 
To: Dean Amir Mirmiran

College of Engineering and Computing

This dissertation, written by $\mathrm{Yu}$ Chen, and entitled A Highly Efficient Biometrics Approach for Unconstrained Iris Segmentation and Recognition, having been approved in respect to style and intellectual content, is referred to you for judgment.

We have read this dissertation and recommend that it be approved.

Naphtali Rishe

Jean Andrian

Armando Barreto

Malek Adjouadi, Major Professor

Date of Defense: November 5, 2010

The dissertation of Yu Chen is approved.

Dean Amir Mirmiran

College of Engineering and Computing

Interim Dean Kevin O'Shea

University Graduate School

Florida International University, 2010 


\section{DEDICATION}

I would like to dedicate this Doctoral dissertation to my parents. There is no doubt that without their continued support and patience I could not have completed this process. 


\section{ACKNOWLEDGMENTS}

I would like to gratefully and sincerely thank my advisor, Dr. Malek Adjouadi, for his guidance, understanding, patience, and most importantly, his friendship. I would also like to give my deepest appreciation to him for teaching me how to succeed with his own example, his incomparable attitude and dedication to work.

My appreciation extends also to my committee members: Dr. Armando Barreto, Dr. Jean Andrian, and Dr. Naphtali Rishe, for the time and efforts they spent advising and encouraging me through this research.

My gratitude also goes to all the members of Center for Advanced Technology and Education (CATE), for the inspiring and interesting discussions and help in my research, and for setting an environment that is conducive to research and learning.

I appreciate the support provided by the National Science Foundation under grants CNS-0540592, CNS- 0837556, HRD-0833093, and CNS-0426125.

Last, but definitely not least, I want to thank the University Graduate School at Florida International University for the support provided through the Dissertation Year Fellowship in the last year of my Ph.D. work. 


\section{ABSTRACT OF THE DISSERTATION \\ A HIGHLY EFFICIENT BIOMETRICS APPROACH FOR UNCONSTRAINED IRIS \\ SEGMENTATION AND RECOGNTION}

by

Yu Chen

Florida International University, 2010

Miami, Florida

\section{Professor Malek Adjouadi, Major Professor}

This dissertation develops an innovative approach towards less-constrained iris biometrics. Two major contributions are made in this research endeavor: (1) Designed an awardwinning segmentation algorithm in the less-constrained environment where image acquisition is made of subjects on the move and taken under visible lighting conditions, and (2) Developed a pioneering iris biometrics method coupling segmentation and recognition of the iris based on video of moving persons under different acquisitions scenarios.

The first part of the dissertation introduces a robust and fast segmentation approach using still images contained in the UBIRIS (version 2) noisy iris database. The results show accuracy estimated at $98 \%$ when using 500 randomly selected images from the UBIRIS.v2 partial database, and estimated at $97 \%$ in a Noisy Iris Challenge Evaluation (NICE.I) in an international competition that involved 97 participants worldwide involving 35 countries, ranking this research group in sixth position. This accuracy is achieved with a processing speed nearing real time.

The second part of this dissertation presents an innovative segmentation and recognition approach using video-based iris images. Following the segmentation stage which delineates the iris region through a novel segmentation strategy, some pioneering experiments on the recognition stage of the less-constrained video iris biometrics have been accomplished. In the 
video-based and less-constrained iris recognition, the test or subject iris videos/images and the enrolled iris images are acquired with different acquisition systems. In the matching step, the verification/identification result was accomplished by comparing the similarity distance of encoded signature from test images with each of the signature dataset from the enrolled iris images. With the improvements gained, the results proved to be highly accurate under the unconstrained environment which is more challenging. This has led to a false acceptance rate (FAR) of $0 \%$ and a false rejection rate (FRR) of $17.64 \%$ for 85 tested users with 305 test images from the video, which shows great promise and high practical implications for iris biometrics research and system design. 


\section{TABLE OF CONTENTS}

CHAPTER

PAGE

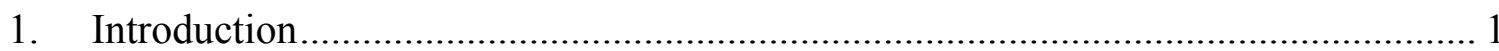

2. Background and Overview of Iris Biometrics ……….......................................... 5

2.1. Introduction to the Uniqueness of Human Iris............................................. 5

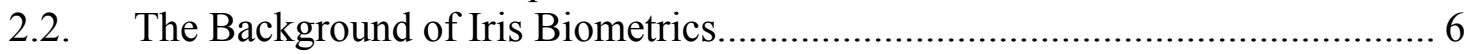

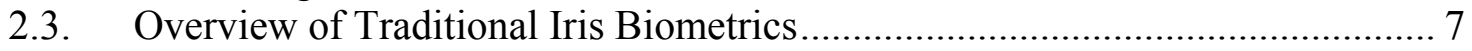

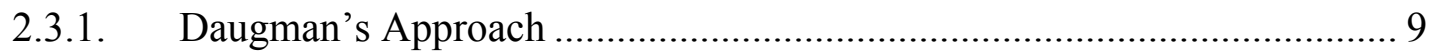

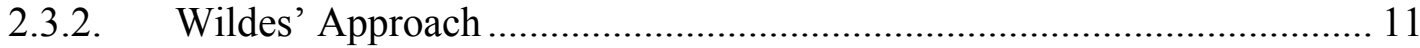

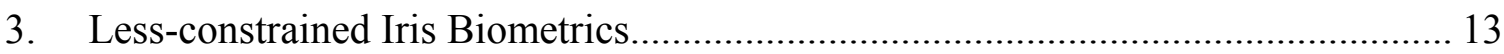

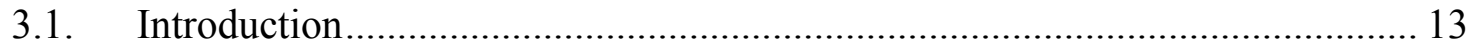

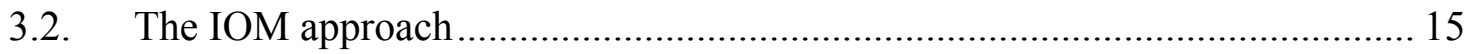

3.2.1. Experimental setup of the IOM system …………................................. 15

3.2.2. Techniques based on the IOM system ................................................. 17

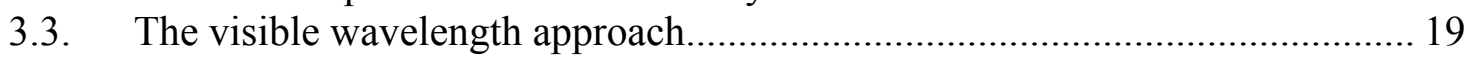

3.3.1. Experimental setup of the visible wavelength approach........................... 20

3.3.2. Techniques based on the visible wavelength system.................................. 21

3.4. Other Less-constrained iris recognition approaches .......................................... 24

4. A New Approach for Unconstrained Iris Segmentation under Visible Lighting

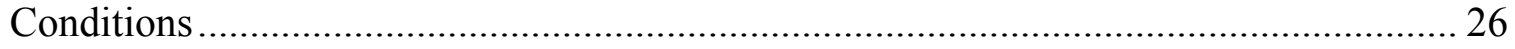

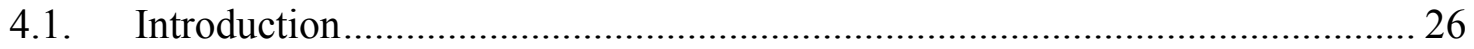

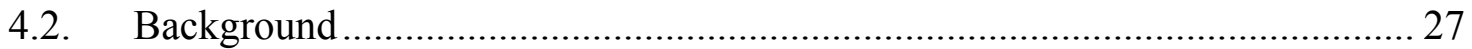

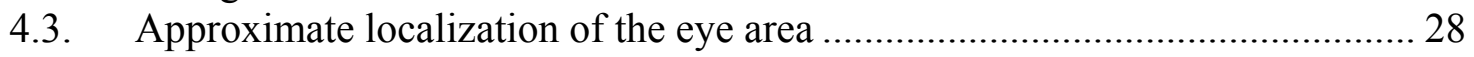

4.3.1. Detecting the sclera area .................................................................... 30

4.3.2. Determining a target area for the eye.......................................................... 31

4.4. Iris outer boundary detection with a fast circular Hough transform.................. 33

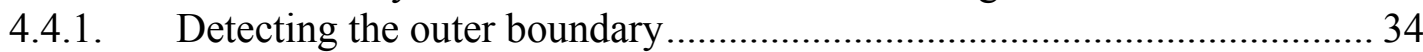

4.4.2. A fast Circular Hough Transform .............................................................. 35

4.5. Detecting the boundary for upper and lower eyelids ...................................... 39

4.6. Circle correction and non-circle boundary detection ..................................... 42

4.7. Pupil, eyelash detection and results reliability verification ............................ 47

4.8. Result and evaluation................................................................................. 49

5. A Robust Segmentation Approach to Iris Recognition Based on NIR Video .......... 53

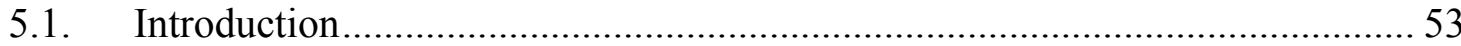

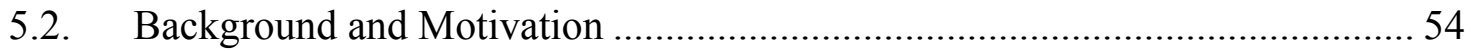

5.3. Eye Images Extraction and Pupil Localization................................................. 57

5.3.1. Eye images extraction from video frames ................................................ 57

5.3.2. Pupil localization and center detection ..................................................... 59

5.4. Defining Outer Boundary and Eyelids.......................................................... 62 


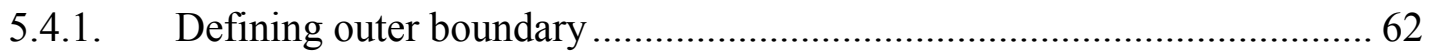

5.4.2. Detecting the Boundaries for the Upper and Lower Eyelids ..................... 64

5.5. Verification and Correction of Outer Iris Boundary ......................................... 68

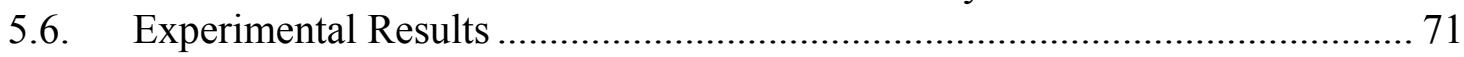

6. Iris Recognition Experiments on Video Images ……........................................... 75

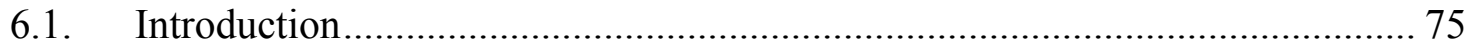

6.2. Optimization in Normalization and Encoding …………............................. 78

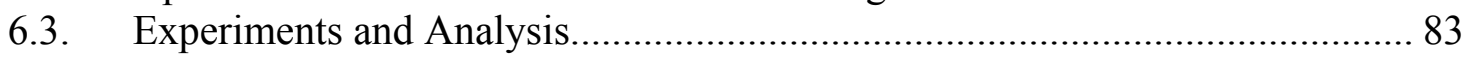

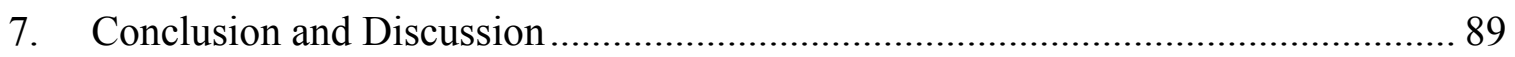

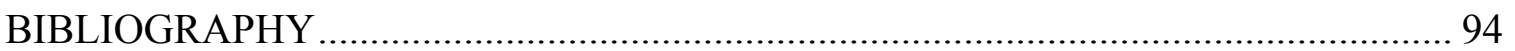

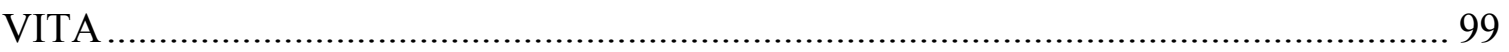




\section{LIST OF FIGURES}

FIGURE

PAGE

Figure 2.1:An anatomical view of the human eye ............................................... 6

Figure 3.1: Image Acquisition Setup of the IOM system ......................................... 15

Figure 3.2 : Eye images acquired from the IOM system and the traditional iris acquisition method. Eye images (a, b, c, d) are extracted from the IOM system, while eye images (c, $\mathrm{d}, \mathrm{e}, \mathrm{f})$ are from the traditional acquisition system as provided by the CASIA database version 3 .

Figure 3.3 :Setup of the image acquisition prototype, signs of A, B in the image denote the two cameras; C,D are the artificial and natural light sources; E is the moving subject. (Image provided courtesy of Dr. Hugo Proença) ........................................... 20

Figure 3.4:Iris image examples captured by the Proença's approach............................ 21

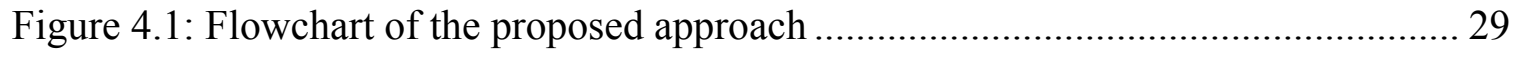

Figure 4.2: Example of sclera detection in the presence of noise pixels ........................ 31

Figure 4.3: Target selection for eye area based on double and single sclera areas.......... 33

Figure 4.4: Example of outer boundary detection using the modified circular Hough transform

Figure 4.5: Example of searching feature points locating on the circular ring around the target circle. 36

Figure 4.6: Average processing time for circular Hough transform using 500 images from UBIRIS.v2 training database 38

Figure 4.7: Example for detecting upper and lower eyelids 40

Figure 4.8: Example of obtaining average gray intensity 41

Figure 4.9: Results of eyelid boundary detection using two different subjects 42

Figure 4.10: Defining the noncircular iris boundary- the intersection of the two circles with the detected eyelids lines is the accrual iris.

Figure 4.11: Relations between the real iris center, original circle center and center for target rectangle 
Figure 4.12: Result examples of proposed circle verification and correction strategy for delineating non-circular iris boundaries ................................................................... 46

Figure 4.13: Process for pupil detection and removal ................................................... 47

Figure 4.14: Examples yielding good results............................................................... 49

Figure 4.15: Examples yielding faulty or undesired results ......................................... 50

Figure 4.16: Examples of detecting the outer iris boundary of rotated iris images ......... 52

Figure 5.1: Eye images acquired from IOM system and traditional iris acquisition method: (a), (b) eye images extracted from the IOM system; (c), (d) eye images from CASIA database (version 3) using the traditional method. 55

Figure 5.2: Noisy eye images acquired from MBGC database : (a), (b) eye images with eyelids occultation; (c), (d) eye images with motion blurs. 56

Figure 5.3: One frame of video and right and left eye images extracted from the frame. (a) frame of subject's NIR video with face appearing, (b),(c) right and left eye images extracted from the frame 58

Figure 5.4: Adaptive histogram methods for defining the pupil. (a) eye image extracted from the video frame; (b) histogram analysis for image gray level intensities; (c) the extracted pupil part after transforming (a) to binary map; (d), (e), (f) demonstrate another case with the same organized order.

Figure 5.5: Sobel edge detection for four directions vs. the Sobel edge detection for horizontal direction: (a) original eye image; (b) resulting edge map using all four directions; (c) resulting edge map using only the horizontal direction.

Figure 5.6: Example of modified Hough circular transform: (a) original eye image; (b) edge map, the center of outer boundary was constrained to be located in the inner circle; (c) edge map, where the edge points of the outer boundary were constrained to be located in the ring area between the two circles; (d) resulting outcome of the modified Hough circular transform.

Figure 5.7: Example of the proposed method for detecting upper and lower eyelids: (a) edge map found with only the vertical direction operator; (b) detected reflection area; (c) edge map after removing undesired edge points; (d) detected points which are on the edge of iris and upper eyelid; (e) detected points which are on the edge of iris and lower eyelid; (f) resulting outcome of the iris detection through eyelids detection. 65

Figure 5.8: Example of obtaining average gray intensity 66 
Figure 5.9: Example for detecting eyelid with more than one line: (a) original edge map; (b) verified edge map before first Hough line transform; (c) edge map before the second Hough line transform towards upper eyelid; (d) final results of the iris extraction process.......

Figure 5.10: Example for defining the noncircular iris boundary. (a) The black circle is the result of the 1st Hough transform; (b) shows the center of pupil and center of original iris center; (c) the square shows the target region which was defined by two centers (d) the white circle is the result Hough circle of target area;(e) shows the intersection of two circles with the detected lines for upper and lower eyelids; (f) is the result after the noncircular correction

Figure 5.11 : Three other examples, (a), (b) show one example which 2 arcs and 3 lines were used together to describe the boundary; (c), (d) show Hough transform can be corrected through this process, the larger circle was the original one obtained from the first Hough transform; (e),(f) show how this method deal with the eye image which is out of video frame, for adjusting the eye image to our segmentation system, we fill the out range part of image with the same pixels of last line or column.

Figure 5.12: An example of successful segmentation: (a) eye image extracted from the video; (b) detected iris boundary; (c) result of the segmented iris; (d) polar transform.

Figure 5.13: Error examples of segmentation results due to other effects: (a) caused by jewelry; (b) caused by heavy eyelids occultation; (c) caused by unexpected reflection; (d) caused by the eye is out of the video frame range;(e) caused by eyeglasses;(f) caused by unexpected reflection; (d) caused by the eye is out of the video frame range;(e) caused by eyeglasses;(f) caused by blurred edge between eyelid and iris..................... 73

Figure 6.1: Process of less-constrained iris recognition based on video ........................ 76

Figure 6.2: Illustration of normalization step with the rubber-sheet algorithm ............... 79

Figure 6.3: Normalization examples for test iris image and enrolled iris image............. 79

Figure 6.4: Iris images before and after contrast enhancement ................................. 81

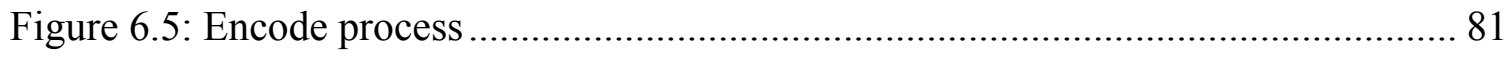

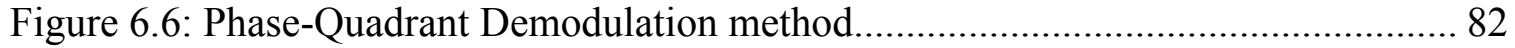

Figure 6.7: Distributions of Hamming Distance for inter- and intra-class comparisons .. 85

Figure 6.8: False Rejected Rate (FRR) and False Accepted Rate (FAR) vs. Hamming Distance. 
Figure 6.9: ROC curves for three cases as the one with histogram transform on the test and enrolled images, the one with the histogram transform on the test images only and

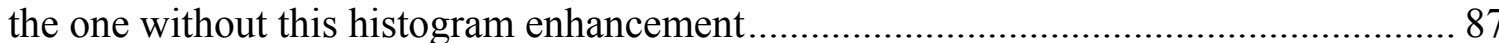




\section{Introduction}

This dissertation establishes a new segmentation method for iris recognition in biometrics applications. Both still images and the less constraining but more challenging video images are used in the experimental evaluation phase. While still images are obtained under all the necessary constraints on the user to obtain high resolution images, video images are less constraining on the users and are obtained while the user is on the move introducing as a consequence several noise sources that need to be addressed and overcome before the iris verification process is carried out.

Biometrics as a discipline has experienced significant growth and carries with it significant practical implications in the realm of security and information access (Daugman 2004). Biometrics provides the most reliable means for personnel identification and verification, as it relies on intrinsic characteristics of individual that are either physiological or behavioral, either which are very difficult to fake or to temper, especially the physiological characteristics (Ross and Jain 2007). These last characteristics include DNA, fingerprints and iris recognition, which is the subject of this dissertation.

To appreciate the significance of the problem at hand in this iris recognition research endeavor, Chapter II provides the background on iris recognition and describe the different image acquisition methods that have been used to this day, from the traditional approach to the more current "Iris on the Move" approach. It lays the background on the issues involved in the most appropriate but yet less constraining approach to image acquisition. This Chapter also provides a balance between image 
resolution along with the inherent noise effects and the need to design a method that is accurate but is also computationally appealing.

Chapter III provides the details on the more challenging methods that impose fewer constraints on the user. These systems are based on the IOM or "Iris on the Move" (Matey, Naroditsky et al. 2006) concept where the image acquisition process is more subtle and convenient to the user. Systems that use Near Infrared (NIR) and visible wavelength approach for the same intent of acquiring video taken at a distance are described in this chapter, The experimental set ups for these different systems are provided to gauge the different issues of noise, illumination, image capture that are needed to be addressed for carrying out effective iris recognition processes. Also, a thorough literature survey is provided on the different image segmentation methods and preprocessing techniques that have been reported in the past. These are detailed as means to provide comparative results with the methods proposed in this dissertation.

Chapters IV and V constitute the core of this research endeavor and are the main contributions of this dissertation. In Chapter IV, a new iris segmentation approach is introduced to deal with eye images acquired from NIR video frames. The most current and relevant database on faces, the Multiple Biometric Grand Challenge (MBGC) (Phillips 2009) database provided by National Institute of Standards and Technology (NIST) is used in the experimental evaluation phase. All the algorithms that have designed towards the segmentation of the iris along with mathematical foundation that supports the different decision making steps are described. These include the processes that allowed for eye images extraction, pupil localization and center detection, defining 
the eyelids as well as the outer boundary between iris and sclera, detecting the boundary for upper and lower eyelids, and the verification and correction of the outer iris boundary. Experimental results based on 149 video and up to 891 total extracted eye images are given.

The issues of coupling computational efficiency with high accuracy in iris detection and extraction are detailed in Chapter $\mathrm{V}$ through a generalized segmentation method which assumes visible lighting conditions. For experimental evaluations that mimic real-world environments, the UBIRIS-II database of noisy eye images is used (Proenca and Alexandre 2005). These are images that are acquired in the less-constrained system allowing for image acquisition while subjects are on the move. The extraction results of the irises are remarkably accurate, exceeding 98\%, and the approach considered was proven to be resilient to the presence of noise. The software developed under this approach placed this research group as the only ranked US team in the top ten of an international competition "Noisy Iris Challenge Evaluation (NICE.I)" that involved 97 participants worldwide, ranking this research group in sixth position (Chen, Adjouadi et al. 2010).

With the segmentation results of the iris, Chapter VI provide an initial attempt at the most complex and ultimate process that of iris recognition, where an individual will be recognized through the segmented iris results. In this chapter the first preprocessing step involves the use of the log polar plot to overcome size and orientation independence, so that the focus is placed rather on the intrinsic characteristics of the iris. Initial results show that only 15 out of 85 individuals that were involved in our experiment where 
falsely rejected, but more importantly none of them were falsely accepted. of the irises under were identified. This is rather impressive for a first round verification process given that the data used is from the NIST-MBGC database which contains realistic noisy images. For subjects on the move at this juncture, the results show an accuracy that led to a false acceptance rate (FAR) of $0 \%$ and a false rejection rate (FRR) of $17.64 \%$ for 85 tested users with 305 test images from the video, which is remarkable considering that we are dealing the less-constrained environment. 


\section{Background and Overview of Iris Biometrics}

\subsection{Introduction to the Uniqueness of Human Iris}

Iris is the colored organ inside the eye. Each human iris has a unique pattern which is known to remain stable throughout a person's life (Daugman 2003). Since the iris is a protected organ inside the human eye (Wildes 1997; Daugman 2001), its intrinsic characteristics cannot be changes either accidentally or intentionally. Because of such reliable characteristics, iris recognition has become a highly dependable and quite accurate among well-known biometric methods such as face identification and fingerprint recognition, among others.

In the human eye, as illustrated in Figure 1, the iris is a thin diaphragm that lies behind the cornea and anterior chamber (Bowyer, Hollingsworth et al. 2008). The muscles of the iris expand and contract the aperture (also known as pupil) within the iris to adjust the amount of light which passes through the lens (Land 1999).

Interestingly, the main structures of the iris begin to form in the third month of gestation, and are completed by the eighth month (Snell and Lemp 1998; Daugman 2004). The growth of iris pigment continues within the first postnatal years, but thereafter the iris remains the same through one's life. Consequently, the structures of the iris are said to be unique to an individual and do remain stable with age.

The overall structure of the iris can be considered to consist of two layers: the anterior layer and the posterior layer. The anterior layer contains the stroma, iris sphincter muscle, iris dilator muscle, and anterior pigment myoepithelium. The iris 
sphincter muscle and dilator muscle contract and expand to reduce or enlarge the pupil size, respectively. The posterior layer of the iris mainly contains the pigmented epithelial cells beneath the anterior layer (Kanski 2007).

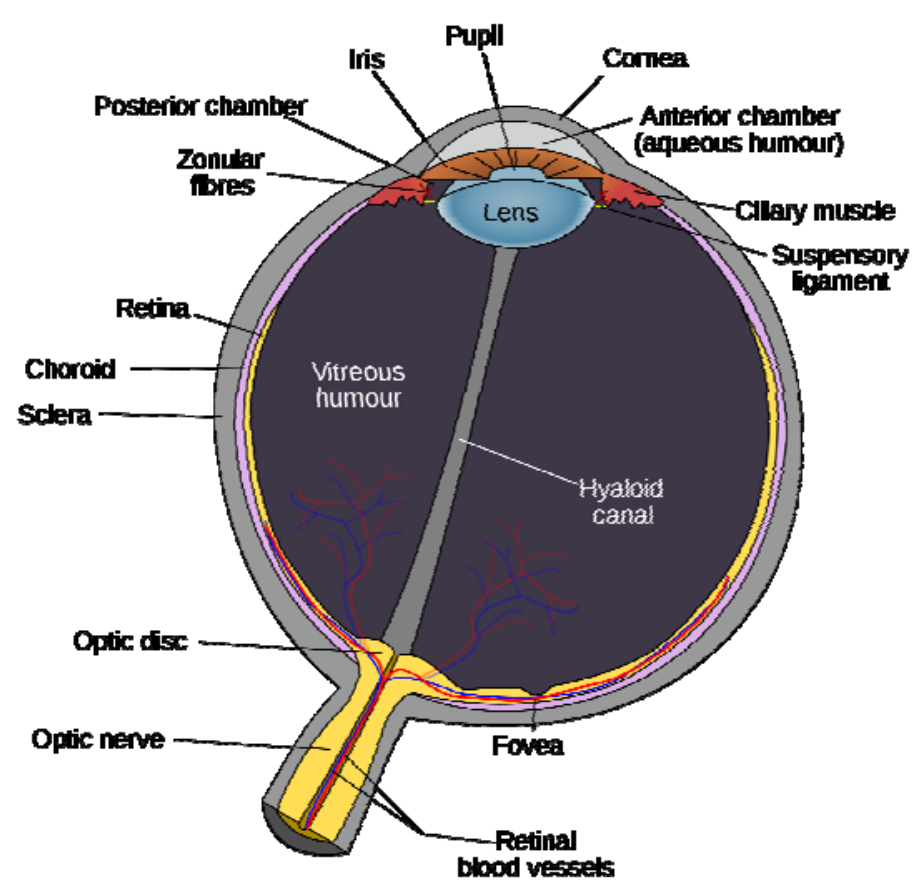

Figure 2.1:An anatomical view of the human eye

\subsection{The Background of Iris Biometrics}

The patterns of the iris are caused by many various features of the anterior layer: the arching ligaments, interlacing ridges, contraction furrows, corona, among others. These features are based on the structure of the anterior layer surface and also the tissues beneath the surface such as blood vessels, and stroma support. The iris color which is usually referred to as the eye color is mainly determined by the anterior melanin pigment. 
The pigment can absorb the short- wavelength light. Thus, if the iris is less pigmented which means that it has less density of the pigment, the iris appears as light colored (grey, blue, green), otherwise, the iris appears dark colored (brown, black) (Davson 1984; Kanski and Menon 2003).

Although the basic structures of the irises are generally common, the pattern of each human iris is distinct. The genetic differences and the uniqueness of the circumstances of each person i.e. the condition in the embryo development give the fact that the detailed pattern of each human iris is unique. Through clinical observation, it has been claimed that even the patterns in the same person's two eyes are different.

Unlike identity verifications which are based on passwords, biometrics relies on intrinsic physical or behavioral characteristics of each person, which evidently do not require that they would be remembered and certainly cannot be stolen. With the rising demands for public safety and national security, biometrics has thus drawn significant attention in recent years. In view of this affirmation, it is an accepted fact, that among all potential biometric methods, iris recognition remain one the most viable and reliable biometric that maybe unrivaled when used for security reasons.

\subsection{Overview of Traditional Iris Biometrics}

Since the first automatic iris recognition system was proposed by J. G. Daugman in 1993 (Daugman 1993), a variety of commercial systems are developed to deal with the eye images and conduct identification or verification processes. Most of the established iris recognition systems rely on four main steps during the iris recognition process, which 
are: iris information acquisition (still image/video), iris segmentation, iris pattern analysis, and iris pattern matching for eventual recognition.

Most of the existing commercial systems adopt near-infrared (NIR) illuminations (with the wavelength between 700 and $900 \mathrm{~nm}$ ) as a source of lighting. It is believed that with NIR illumination, the iris shows more detailed patterns as the iris tissues absorb the short-wavelength light and let the long-wavelength go through. During the iris acquisition step, the user (object) is usually still and looks at the camera, and the acquisition system access the focus of the iris and automatically adjust the camera to take the iris images or videos.

The iris segmentation step isolates the iris region from the eye images. Some of the segmentation strategies consider the boundaries of the pupil and the iris to be circular in shape. The circles of pupil and outer iris (limbic) boundaries are detected in order to localize the iris which is the part within these two circles. In this step, noise reduction methods are used to attenuate to some degree the inherent noise effects, and preprocessing methods are used to deal with occlusions caused by eyelids, eyelashes, and reflections.

In the iris pattern analysis step, various filters are used by most iris recognition systems to encode the texture of the iris. The decision of iris verification and identification is generated in the iris matching step. In the matching step, the distances between codes generated for the irises are calculated, and a threshold of the distance is usually used to determine if any two codes belong to a same iris. For the application of verification, the system would provide a degree of similarity in subjects' codes within a stored database. For the identification process, the system would identify the subject 
based on whether the degree of similarity has crossed a set threshold that guarantees the highest degree of certainty in comparison to all the stored iris codes in the database.

Among most of the established iris recognition systems, Daugman's approach and Wildes' approach remain the most important and most well-known. Nearly all other methods that have been proposed since were developed using the fundamentals steps outlined in the pioneering work of Daugman and Wildes.

\subsubsection{Daugman's Approach}

The concept of the automatic iris biometrics system was first proposed in the patent by Flom and Safir in 1987(Flom and Safir 1987), but it lacked any actual implementation. Some of their proposed ideas resemble today's established iris biometrics approaches, such as using the image acquisition system to acquire iris information, and using the pattern recognition algorithms to encode the iris image.

The first practical automatic iris pattern encoding and recognition method was proposed by Daugman in 1993. Since then, the basic idea of Daugman's original approach inspired many of the new research developments as well as commercial

products (Daugman 2007; Bowyer, Hollingsworth et al. 2008). In his approach, the monochrome cameras with NIR illumination lighting sources are used in the iris acquisition step. The total high-frequency power in the 2D Fourier spectrum of each captured image/frame is calculated to access the focus of the iris. The camera is adjusted to focus on the iris and a feed-back (voice or vision signal) is given to help the user position his/her head within the camera's field of view. The pupil boundary and limbic boundary are treated as two non-concentric circles. Each circle is detected separately and 
described by their center coordinates and radius. An integro-differential operator is used to search for the boundary circles. This particular integro-differential operator is defined as:

$$
\max _{\left(r, x_{0}, y_{0}\right)}\left|G_{\sigma}(r) * \frac{\partial}{\partial r} \oint_{r, x_{0}, y_{0}} \frac{I(x, y)}{2 \pi r} d s\right|
$$

where $I(x, y)$ is an intensity value of the image domain $(x, y)$. This equation seeks the maximum value in the blurred partial derivative with respect to increasing radius $r$, along an arc denoted by $d s$ of a circle with center coordinates $\left(x_{0}, y_{0}\right)$ and radius $r$. The $G_{\sigma}(r)$ is a Gaussian smoothing function of a scale set by $\sigma$, and the symbol $(*)$ refers to the convolution operation.

The upper and lower eyelids are modeled as two arcs, and the arcs are detected with this integro-differential operator.. The difference is the path of contour integration in the operator that is changed from circular to arcuate.

Because the image sizes of the irises are different and the degrees of pupil dilations vary based on the illumination, a normalization method is used to transform the segmented iris image to a normalized polar space. In the normalized polar space, every pixel in the iris image is represented by an angle between 0 and 359 degrees and a radial coordinate between 0 and 1 .

A two-dimensional (2D) Gabor wavelet filter is then used to extract the textures from the normalized iris image. The encoded texture is represented as a binary code and compared with stored iris codes to generate a final recognition decision. During the comparison, the Hamming distance is calculated to measure the difference between two 
iris codes. A smaller distance indicates a better confidence of the similarity between the two iris codes. A threshold of the distance would be used to decide if the two iris codes belong to the same iris or not.

It is reported that Daugman's approach achieves excellent accuracy results on the basis of a threshold Hamming distance of 0.330 over 2.3 million comparisons between different irises.

\subsubsection{Wildes’ Approach}

The Wildes' approach is also very prominent in the field of iris recognition. It uses different image acquisition and iris segmentation processes which gives it some advantages over Daugman's system in some aspects (Fancourt, Bogoni et al. 2005).

In the acquisition system, a more complicated set-up, which includes the so-called "diffuse source and polarization in conjunction with a low light level camera" is used to eliminate the reflection spots (Wildes, Asmuth et al. 1996; Wildes 1997). In the iris segmentation step, the circular Hough transform is used to detect the pupil and limbic boundaries. The Hough transform is known to be tolerant to gaps in edge descriptions and is relatively unaffected by image noise. The well-known circular Hough transform is often defined as:

$$
H\left(x_{c}, y_{c}, r\right)=\sum_{j=1}^{n} h\left(x_{j}, x_{j}, x_{c}, y_{c}, r\right)
$$

where $h\left(x_{j}, x_{j}, x_{c}, y_{c}, r\right)=1$ if the edge point $\left(\mathrm{x}_{\mathrm{j}}, y_{j}\right)$ is on the circle with center $\left(\mathrm{x}_{\mathrm{c}}, y_{c}\right)$, and radius $r$; otherwise, $h\left(x_{j}, x_{j}, x_{c}, y_{c}, r\right)=0$. With such a circular transform, each edge point in the image space votes for each possible circle passing it in the parameter space. 
The maximum value of $H\left(x_{c}, y_{c}, r\right)$ indicates the target circle which has a center at $\left(\mathrm{x}_{\mathrm{c}}, y_{c}\right)$ and a radius of $r$.

In the iris pattern analysis step, a Laplacian of Gaussian (LOG) filter with multiple scales is used to analyze the iris texture, and the filtering results are compared without binarizing to a compact representation as is the case in the Daugman's approach. Thus, more feature details may be extracted and compared when using the Wildes' method.

It is claimed that Wildes' approach achieved 0 false accept rate and 0 false reject rate, based on a database of 600 iris images from 60 different persons. 


\section{Less-constrained Iris Biometrics}

\subsection{Introduction}

One of the key problems of conventional iris recognition methods is that they are based on processing a single iris image at a time and require good image quality as an essential initial condition (Matey, Naroditsky et al. 2006; Proenca, Filipe et al. 2010). These requisites entail considerable constraints on users for taking such iris images. Under the current technological setups, and in order to take an adequate iris picture, the subject must be still, look directly at the camera and in a direction that satisfy strict illumination controls (Chen, Adjouadi et al. 2010). This process is obviously too constrained, time-consuming, and often inconveniences the subject.

With the increasing demands in public safety and security and with the demands necessitated by classified information access and other security measures, good identification processes are becoming a requisite for seeking high accuracy but with a fast and reliable outcome (Zhou, Du et al. 2009; Matey, Broussard et al. 2010). Thus an effective iris recognition method is one that should initially overcome the rigid constraints imposed during iris image acquisition, and offer fast processing speed.

In all of the four steps of iris recognition, the iris information acquisition step which captures the iris images or videos of the users (subjects) is by far the most restrictive. It is this initial and only step that needs complete users' cooperation. Thus, the most distinguishing difference between traditional iris recognition and unconstrained iris recognition is in the set-up of the iris acquisition system to free the user from any undue 
constraints. Thus, unconstrained iris biometric systems must be able to process iris images or video with somewhat low image quality.

Amongst most of the conventional iris recognition systems, iris acquisition is the most time-consuming step and is considerably inconvenient to users. To obtain the iris images with required ideal or demanding qualities for traditional iris recognition system, various rigid constraints are imposed on the subject's stands, head alignments, movements, light illuminations, etc.. Most of the unconstrained iris recognition systems benefit from their innovative endeavors in the more convenient iris acquisition setups. Among those, the "Iris on the Move" (IOM) system and visible wavelength systems are the two most well-known and promising prototypes.

Because of the innovative iris acquisition setups, the characteristics of the iris images captured by less-constrained iris recognition systems are different and more challenging to process, compared with those obtained from the more controlled conventional iris recognition systems. Most challenging are the severe noise effects that are inherent to these unconstrained iris recognition systems. Typical sources of noise include motion blur, defocus, eyelashes or eyelids obstruction, specular reflection, among others. Those noise effects could make it impossible for the system to normalize, encode and generate accurate matching results. Thus, new alternate strategies and algorithms are proposed by the biometrics research community to compensate for those newly introduced difficulties and obtain acceptable recognition performances under unconstrained environments. The rest of this section gives an up-to-date overview of unconstrained iris recognition systems and related techniques associated with them. 


\subsection{The IOM approach}

The Iris on the Move (IOM) that was first introduced by (Matey, Naroditsky et al. 2006) was based on Near Infrared (NIR) video taken at a distance, with its targets being moving subjects (Matey, Naroditsky et al. 2006). One of the significant merits of the IOM system is that it allows for iris identification or verification even when a subject is walking at a normal pace at a speed of up to $1 \mathrm{~m} / \mathrm{s}$.

\subsubsection{Experimental setup of the IOM system}

The scenario for iris recognition under the IOM system is as illustrated in Figure 3.1. The prototype uses three high resolution video cameras towards a portal at a distance of about 3 meters. The NIR illumination sources are embedded within the portal. The NIR video is taken when the subject walks through the portal, and the subject could be wearing eyeglasses or contact lenses.

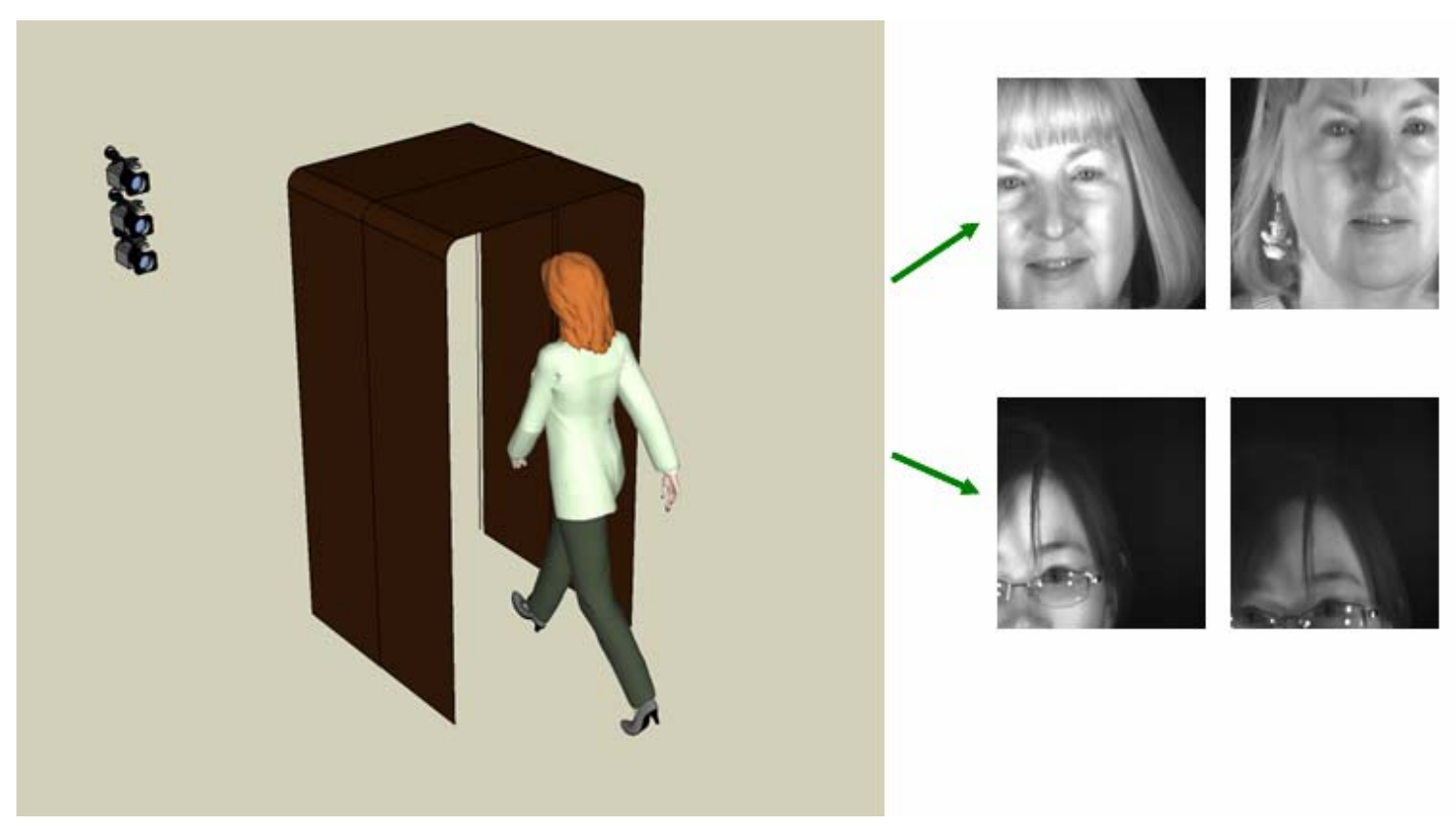

Figure 3.1: Image Acquisition Setup of the IOM system 
For iris verification or identification, the NIR videos of subjects' faces are taken with a resolution of $2048 * 2048$. The iris images acquired from these videos are different from those acquired through traditional acquisition methods. For most of the traditional methods, the total number of pixels across the iris is usually more than 200, but eye images from the videos only offer about 120 pixels across the iris part.

The subject to be pictured should be at an ideal distance for the fixed focus video camera to take the desired frames of the subject's face. Through their study, Matey et al. claim that those frames which are taken with a depth of field at about $12 \mathrm{~cm}$ yield the perfect focus distance which can be accepted for iris verification. While conducting iris verification with the IOM system, the video is taken at 15 frames per second and the subject's walking pace is allowed to be approximately $1 \mathrm{~m} / \mathrm{s}$. Consequently, there are no more than 2 frames that can be captured within that $12 \mathrm{~cm}$ depth of field. For subjects that are on-the-move, the iris images extracted from limited frames may suffer from various kinds of noisy effects caused by off angle, motion blur, occultation caused by eyelid or eyelashes, and unexpected light reflections.

To appreciate the complexity of the problem, some examples of iris images obtained from the IOM system and from the traditional system are provided in Figure 3.2 for comparative purposes. 


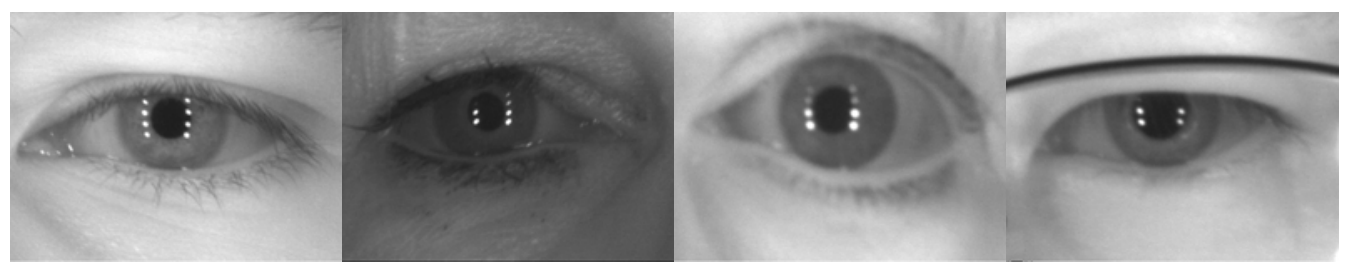

(a)

(b)

(c)

(d)

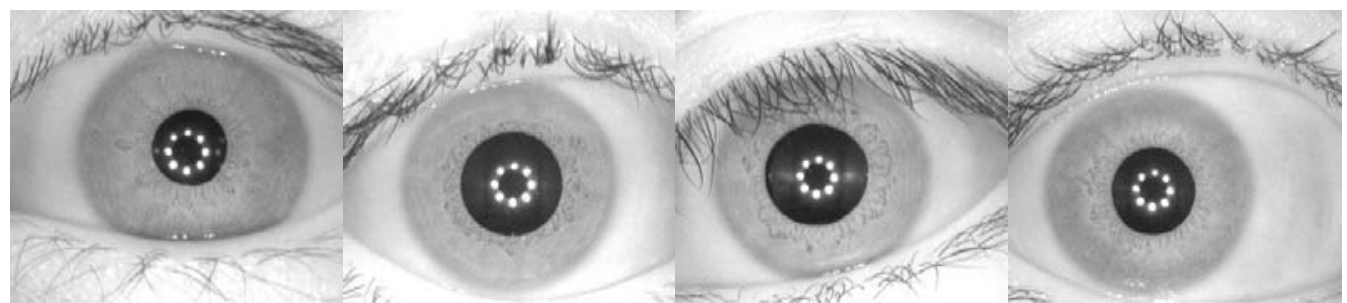

(e)

(f)

(g)

(h)

Figure 3.2 : Eye images acquired from the IOM system and the traditional iris acquisition method. Eye images ( $a, b, c, d)$ are extracted from the IOM system, while eye images (c, d, e, f) are from the traditional acquisition system as provided by the CASIA database version 3

\subsubsection{Techniques based on the IOM system}

Matey et al. adequately proved the feasibility of the IOM system. Through their experiments, it is claimed that the overall recognition rate for 119 subjects is $78 \%$ and with some improvement on the acquisition process, the success rate could rise up to as high as $99 \%$. They conclude that the IOM system "can capture iris images of recognition quality from subjects walking at a normal pace through a minimally confining portal”.

The detailed segmentation approach for IOM system is proposed by Chen et al. (Chen, Wang et al. 2008). Their proposed approach starts from the eye image extraction which is based on the reflections on the eyes generated by the illumination system. An adaptive histogram method is used to detect the pupil. The searching of limbic boundary and eyelids benefits from the obtained pupil information. The outer boundary of the iris 
was detected by a modified Hough circular transform. A new eyelids and eyelashes detection method was introduced in this approach to overcome the noise from such unconstrained images, which do affect the detection process. In their approach, the pixels which are on the edge between iris and eyelids or eyelashes will be detected first, and then the Hough transform would be performed. Their experimental results based on the MBGC database show the approach to yield a 93\% segmentation accuracy rate.

Lee et al. proposed an "object detector" algorithm based on the Viola-Johns method combined with a modification made by Lienhart-Maydt to detect the eye in the NIR video (Lee, Phillips et al. 2008). Then, an automatic segmentation algorithm based on the Wildes' method is used to localize the iris. The feature extraction is conducted by a 1D Log-Gabor filter. The Hamming Distance (HD) measure is used in the matching stage. Their experimental results shows that an eye detection accuracy of $97.69 \%$ and of $81.50 \%$ in the iris region segmentation process. The matching rate is reported to be greater than $54 \%$.

An iris image evaluation method is proposed by Zhou and his colleagues to select the extracted NIR iris images with acceptable quality for recognition in the IOM system (Zhou, Du et al. 2009). Their image evaluation strategy includes: a so-called quality filter unit to delete the low-quality images after image extraction; a segmentation evaluation unit and a segmentation scores unit to evaluate the segmentation accuracy; and a score fusion unit to combine the quality and segmentation measurements to generate a confidence score in the recognition stage. The two-dimensional Gabor wavelet method and the 1-D-Log-Gabor wavelet method are used for the recognition stage. They claim that with the proposed image evaluation method, the genuine acceptance rate (GAR) 
increases by $407.1 \%$ and $216.8 \%$, respectively, in the left and right eyes, compared with the GAR obtained with traditional methods at the same false accepted rate of $10^{-4}$.

\subsection{The visible wavelength approach}

One of most difficult and important issue for the design of a less-intrusive iris recognition system is the trade-off between the demanding irradiance and the illumination safety requirements for the eyes. Being able to capture iris images of the subjects with on-the-move and at-a-distance conditions, the acquisition system needs to achieve larger depth-of-field with considerable short exposure time. Thus, increased intensity of irradiance would be necessary for the optical system in the acquisition step. It is indicated that the use of visible wavelength illumination would be better and safer to achieve such a lighting requirement. Different with the NIR illuminations, the visible wavelength illumination makes people react instinctively, as a safety measure, with eye blinking, pupil contraction/dilation, and evasion, if the illumination happens to be intense. A Visible Wavelength (VW) prototype approach was proposed by Proença et al. in University of Beira Interior (Proenca 2010; Proenca, Filipe et al. 2010). And more research interests have currently been drawn to this idea.

With the VW system the iris images can be also taken when the users are at a given distance. The system can also capture iris images when users are on the move and with different pose or head alignments. Furthermore, the system uses the visible light instead of the NIR illuminations in order to offer better safety for the users. 


\subsubsection{Experimental setup of the visible wavelength approach}

The experimental setup of the iris acquisition adopted in the visible wavelength approach is as illustrated in Figure 3.3. The commercially available camera (Canon EOS 5D in the proposed prototype) is used as the iris image capture device. The acquisition system is designed to function in an environment without rigid illumination control. In their prototype, the setup of the imaging system works under both natural light source and visible artificial light source (Proenca, Filipe et al. 2010).

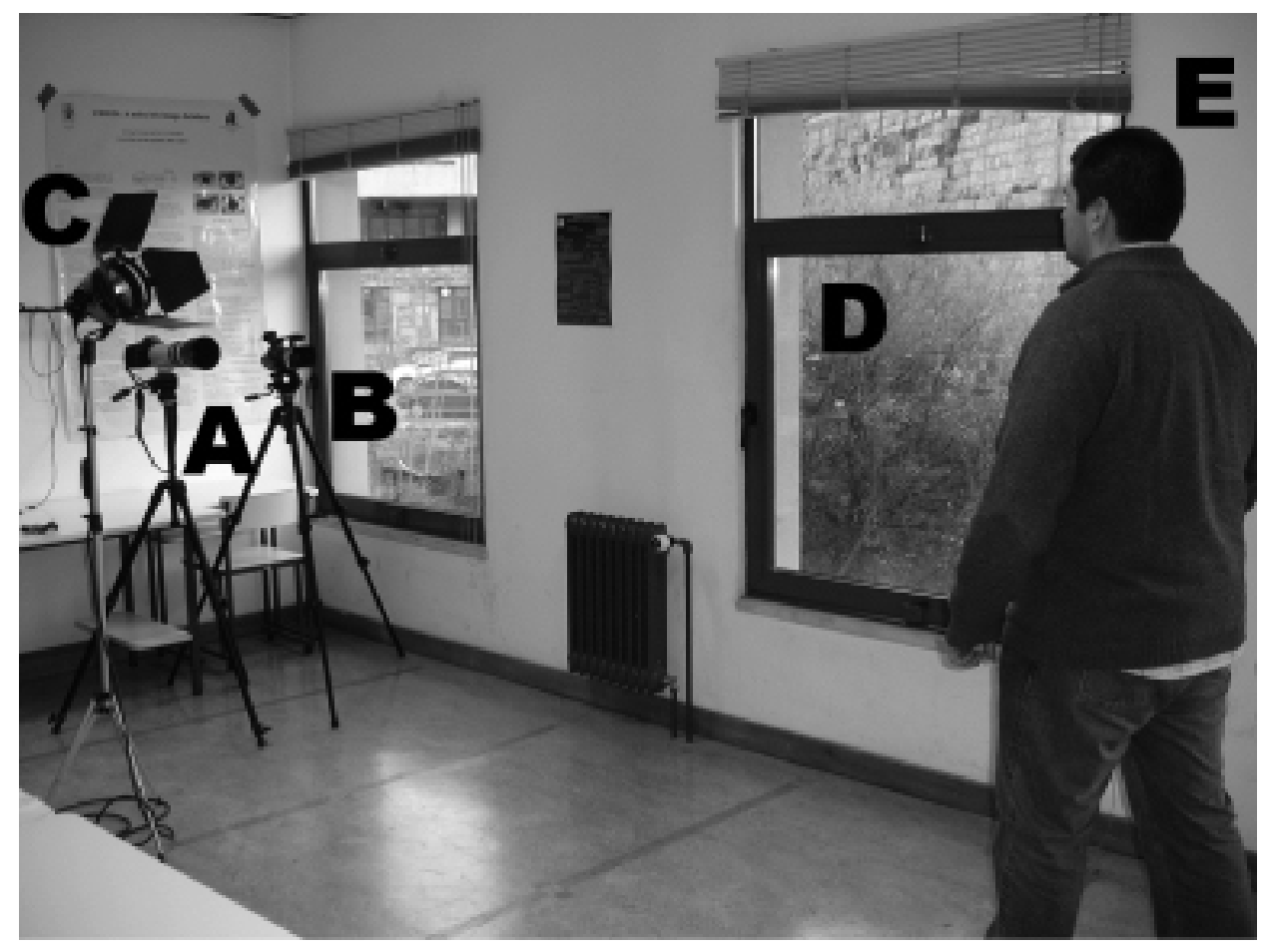

Figure 3.3 :Setup of the image acquisition prototype, signs of A, B in the image denote the two cameras; $\mathrm{C}, \mathrm{D}$ are the artificial and natural light sources; $\mathrm{E}$ is the moving subject. (Image provided courtesy of Dr. Hugo Proença)

The system is claimed to be able to capture multiple iris images when the subject is walking at a slightly slower pace within a range of distances between four and eight meters (Proenca 2010). With such an imaging system, the proposed iris recognition 
system can tolerate occasional effects of movements such as blinking, turning the head, and looking sideways.

\subsubsection{Techniques based on the visible wavelength system}

Because images are taken with less-constrained conditions on illumination, subject's movements, poses, and head alignments, the iris images captured with the Proença's acquisition system generate much more realistic noise conditions, such as specular reflections, off-angle situation, including the wearing of glasses. Some example images are shown in Figure 4. Besides noise effects, the visible wavelength characteristic of iris images show substantial differences between the iris images captured by visible wavelength system and images obtained with traditional iris recognition systems. Because of such differences, conventional iris segmentation approaches have not yet been able to process visible wavelength iris images; and hence a lot of research efforts have been made with the focus on the iris segmentation stage towards more effective visible wavelength-based systems.
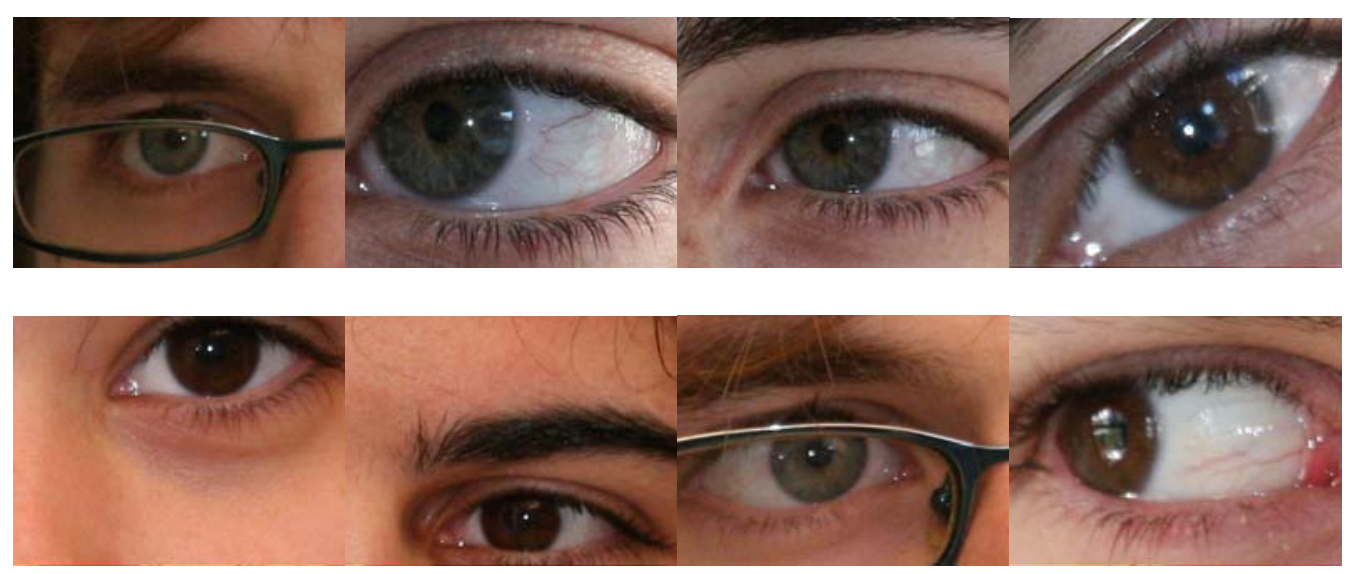

Figure 3.4:Iris image examples captured by the Proença's approach 
A segmentation approach starting with a clustering based coarse iris localization method is proposed in (Tan, He et al. 2010). The performance of this approach greatly benefits from this novel initial processing step. The Specular reflection removals followed by a region growing strategy are conducted to cluster the iris image into coarse iris region and skin region. Then, with the considerations of semantic priors, the iris region is refined and non-iris regions including eyelashes, eyebrow, eye glasses, and hair, are also detected. Also, an enhanced integro-differential operator, just like in the Daugman's approach, is used to detect the pupil and limbic boundaries. The eyelid is detected by a 1-D horizontal rank filter, adopting an eyelid curvature model. In the final step, the eyelash occlusions are excluded, using parametric models based on the intensity statistics of different iris regions.

In the approach proposed by Sankowski et al., the specular reflections are detected and filled in a YIQ color space (Sankowski, Grabowski et al. 2010). Then, again the conventional integro-differential operators are used to detect the pupil and limbic boundaries. Finally, parametric models based on the properties of the eye image are used to describe the upper and lower eyelids.

A knowledge-based segmentation approach was later proposed by Almeida, an idea that was inspired by the paradigm of expert systems (de Almeida 2010). Thus, a set of rules are defined to drive the system as a 'human expert'. Those rules are set based on the perceived natural properties of the human eye such as the following semantic priors: "the pupil should be a very dark small circle", "the centers of the pupil and the iris should be close", "region around the pupil should have a reasonable color of iris", etc. The 
approach consists of the following steps: image pre-processing; pupil and iris localization; and the combination of pupil, iris, and eyelids detection.

The so-called AdaBoost algorithm was adopted in both of the segmentation approaches proposed by $\mathrm{Li}$ et al. and Jeong et al. to detect a coarse location of the eye (Jeong, Hwang et al. 2010; Li, Liu et al. 2010). In Li et al.'s approach, a novel limbic boundary identification strategy is proposed. It uses K-Means clustering based on the cooccurrence histogram to localize the limbic boundary. They use a new parabolic integrodifferential operator combined with a RANSAC (Random Sample Consensus)-like method to detect the upper eyelid boundary. On the other hand, in Jeong et al.'s approach, they adopt the color information of the image for detecting the obstructions caused by the image ghosting effects. The identification of the corneal specular reflection is used to determine if there is an "open eye" appearing in the image.

With the same intent, Chen et al. proposed an approach to achieve both accurate segmentation and fast processing speed (Chen, Adjouadi et al. 2010). The proposed approach relies on an effective search for the sclera area of the image. A threshold of saturation value of the HSI color model is obtained by calculating the biggest group derivative of the original color image histogram. A binary map is then generated to indicate the sclera area. The method determines a more refined target area in order to accelerate the circle searching for the outer iris boundary. The outer boundary of the iris was detected using a very fast and accurate modified circular Hough transform. The linear Hough transform is then used recursively to extract the edges of eyelids. A novel new method of verification and correction for the non-circular outer iris boundary is also 
developed. It is claimed that their approach achieves accuracy higher than $97 \%$ with an execution speed of $0.83 \mathrm{~s}$ per image, which is quite significant.

Labati et al. applied an intro-differential technique to roughly localize the center and radius of the outer iris. The search region for pupil is significantly reduced by this estimation method (Labati and Scotti 2010). Polar-transformation is used to linearize the estimated region of iris and pupil boundaries. Thus, two obtained image strips containing iris boundaries are processed to define the accurate location of the pupil and the outer iris boundary of the iris.

The recognition process remains in the discussion stage among the biometrics community, due to the extreme challenges posed by illumination, capturing distance, and subject movement among others. However, Proenca et al. proved the feasibility of the recognition process by applying Daugman's traditional recognition strategy with manually segmented color iris images (Proença 2009; Proenca 2010). They selected 1000 segmented good quality iris images obtained from visible wavelength system as templates. Then, they compared them with 10,000 non-iris or partial iris images and 10,000 natural and synthetic texture images, generating a false match rate at $P(s<0.33) \approx 1.03 \times 10^{-12}$, which can be considered to be negligible. They point out that the visible wavelength iris recognition system has potential to produce an extremely low false match rate, which is favorably in biometrics applications.

\subsection{Other Less-constrained iris recognition approaches}

Wheeler et al. present a minimal-user-intrusive iris recognition system (Wheeler, Perera et al. 2008). Their work is focused on the development of an automatic iris 
acquisition system to the convenience of the users. The system uses a pair of fixed widefield-of-view (WFOV) surveillance cameras to detect the head position with stereo vision. A pan-tilt head is used to direct the NIR illuminators and the iris camera to focus on the subject. Thus, the system allows the user to stand in front of the camera and faces it for carrying out the iris verification process. A classic Daugman-style approach is used to perform the segmentation and recognition processes. They claim that, with the use of a normalized Hamming Distance of 0.3 , their system achieves the verification false recognition rate of $10^{-6}$, and the whole iris identification process can be completed in $3.2 \mathrm{~s}$ on an average.

Furthermore, an iris image deblurring method is proposed by Huang et al. to improve the quality of the images with defocus or motion blur defects for lesscooperative iris recognition systems (Huang, Ren et al. 2009). Since in the lessconstrained iris recognition scenarios, the user is usually allowed to walk or move, the movement or defocus degrades the image quality significantly. Thus, the intent in this study was to improve the recognition process by introducing a deblurring method. They apply a depth sensor in the iris acquisition system to obtain the 3D depth information. Their deblurring algorithm is based on the depth information and prior knowledge on the iris image. Although their segmentation and recognition algorithms are not fully detailed for a thorough assessment, they claim that the application of the deblurring method can reduce the mean of the authentic distribution by $12 \%$. 


\section{A New Approach for Unconstrained Iris Segmentation under Visible Lighting Conditions}

\subsection{Introduction}

A robust and fast segmentation approach towards less constrained iris recognition under visible lighting conditions is introduced in this chapter. The UBIRIS.v2 database (the second version of the UBIRIS noisy iris database) is used to examine the proposed approach. The iris images in the UBIRIS.v2 are captured from the unconstrained iris acquisition setup introduced by Hugo Proença and colleagues (Proenca, Filipe et al. 2010). Such an inventive iris acquisition method with less constrained image taking conditions under visible lighting can impose minimal to no constraints on the iris verification and identification process as well as on the subject. Consequently, to provide acceptable accuracy measures, it is critical for such iris recognition system to be complemented by a robust iris segmentation approach to overcome various noise effects introduced through image capture under different unconstrained recording scenarios.

The proposed algorithm consists of five implementation steps which include:

(1) localizing approximately the eye area of the noisy image captured at (normal) visible wavelength using the extracted sclera area;

(2) defining the outer iris boundary which is the boundary between iris and sclera;

(3) detecting the upper and lower eyelids;

(4) conducting the verification and correction for outer iris boundary detection; 
(5) detecting the pupil area and eyelashes and providing means for verification of the reliability of the segmentation results.

The results demonstrate that the accuracy is estimated as being $\geq 98 \%$ when using 500 randomly selected images from the UBIRIS.v2 partial database, and was estimated at $\geq 97 \%$ by an independent group in a "Noisy Iris Challenge Evaluation (NICE.I)" in an international competition that involved 97 participants worldwide, ranking this research group in sixth position. This accuracy is achieved with a processing speed nearing real time.

\subsection{Background}

An effective iris recognition method is one that overcomes the rigid constraints imposed during the iris image acquisition process (Proenca and Alexandre 2006), and still offers near real time processing (Bowyer, Hollingsworth et al. 2008).

The UBIRIS.v2 partial dataset, which contains 500 static eye images with more realistic noisy effects as provided by the University of Beira Interior, was used to assess the merits of this method. The noisy iris images are all captured under the Visible Wavelength (VW) iris acquisition prototype proposed by Hugo Proença and his colleagues. The setup details of such iris acquisition system were provided in Chapter 3 of this dissertation. It is indicated that the use of visible wavelength illumination would be safer and more economical to achieve unconstrained iris biometrics (Proenca and Alexandre 2010). With visible wavelength lighting sources, the iris acquisition equipment and the image taking process do not need to be adjusted for the NIR lighting. Thus, the 
costs to use visible wavelength iris biometrics would be lower than using the NIR-based iris biometrics.

Nonetheless, serious noise effects remain inherent to the visible wavelength iris recognition systems (Proenca 2010). Typical sources of noise include motion blur, defocus, eyelashes or eyelids obstruction, specular reflection, among others. Traditional segmentation methods do not perform well under such conditions (Proenca and Alexandre 2010). Thus, a good segmentation approach which can extract useful iris information from noisy iris images would be a crucial first step. Consequently, the focus of this work is first placed on the development of a robust segmentation approach that can overcome such unpredictable noise effects in order to secure recognition outcomes with a high accuracy. This first research step is complemented with efforts to optimize the processing speed seeking near-real-time. Later, the executable program of the proposed method was also submitted for independent testing through an international contest “Noisy Iris Challenge Evaluation (NICE.I)” . The approach introduced here ranked us in $6^{\text {th }}$ position out of 97 competitors worldwide, with due consideration to both accuracy and near real-time processing.

\subsection{Approximate localization of the eye area}

There are five major stages contained in the proposed approach, as illustrated in the flowchart given in Figure 4.1. 


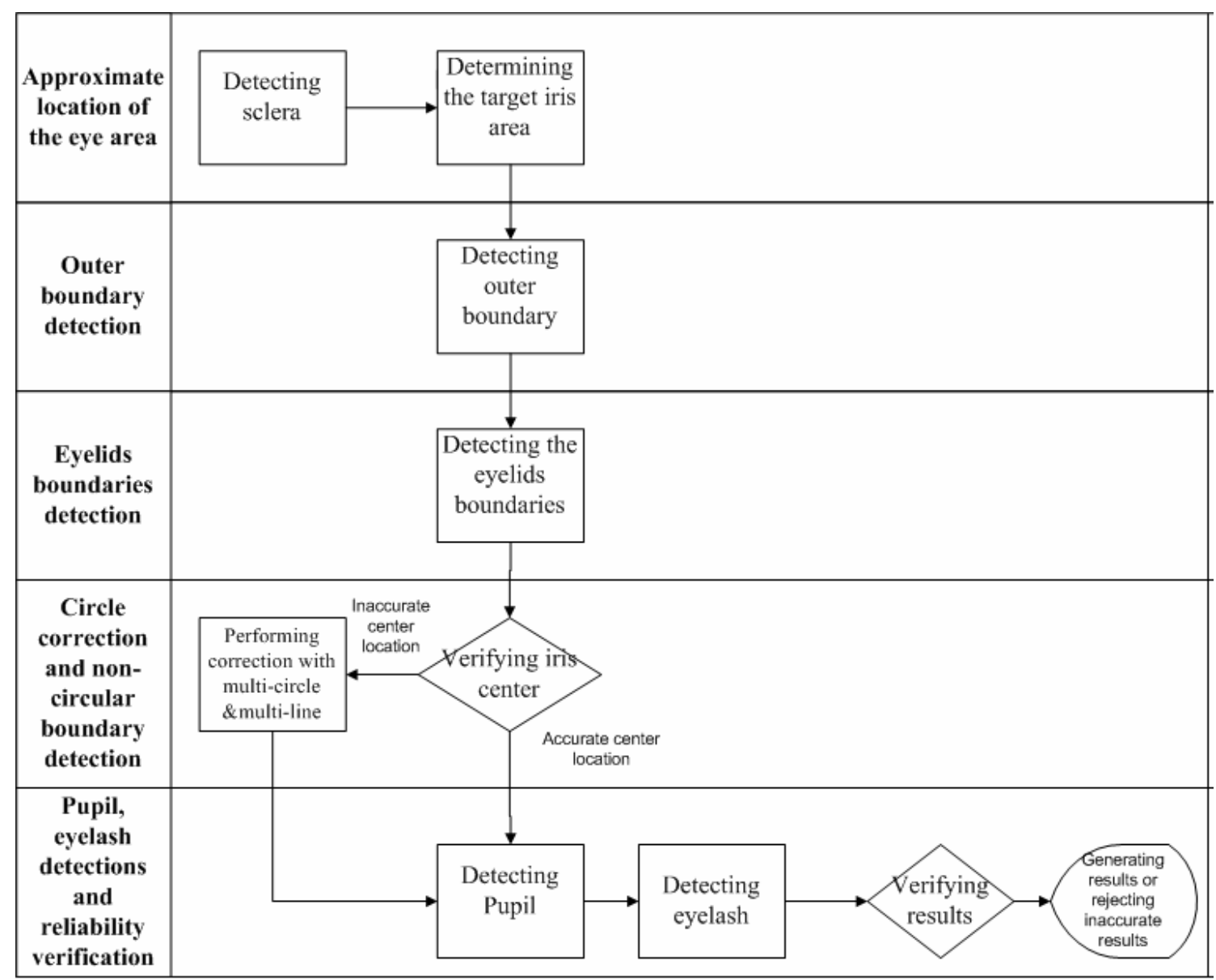

Figure 4.1: Flowchart of the proposed approach

The images from the UBIRIS.v2 database were taken with the subject on the move and at different distances (between 3 and 7 meters). Thus, the irises of the images may vary significantly in size and location. For the provided UBIRIS.v2 training dataset, the amount of pixels across the iris ranged from 75 to 190, and the iris may appear in any region within the close-up image. Therefore, as an initial step in the proposed approach, a method is used to approximately localize the eye part of the image, in order to obtain a smaller and more refined target area. This method, which is of considerable benefit in seeking accuracy and fast processing speed for segmentation purposes, consists of two 
steps: (1) finding an approximate eye area based on sclera detection, and (2) determining an adaptive target to confine the search for the iris.

\subsubsection{Detecting the sclera area}

Unlike conventional iris images, which are mostly captured under NIR (near-

infrared) wavelengths with rigidly constrained environments, all the close-up images from the UBIRIS.v2 training dataset are full-color images taken under visible wavelength. For the UBIRIS.v2 dataset, the pupil area may not always appear darker than other parts, especially for images containing heavily pigmented (dark) irises or images with serious noise effects. Thus, the pupil on the image may no longer be the proper starting point to find the eye area. But through analyzing the full-color images, the sclera parts are commonly found to appear less saturated (white) than other parts of the images. Consequently, the sclera area is used instead for determining the target eye area.

The HSI color model decouples the intensity component from the color-carrying information (hue and saturation), where the saturation value of the HSI model refers to the degree of how much the white was added to the color (Shapiro and Rosenfeld 1992; Gonzalez and Woods 2008). Thus, the saturation value of the HSI model is more tolerant to noise effects for detecting the sclera area. The saturation value can be computed from the RGB values using Eq. (1).

$$
S=1-\frac{3}{(R+G+B)}[\min (R, G, B)]
$$

For the saturation value of the HSI model, when the pixel is white, $S=0$; when the pixel is pure colored, $S=1$. Through our experimental analysis, the saturation values 
of sclera areas provided a range for $S$ between 0 and 0.21 . The saturation value, which refers to sclera, would be calculated as a threshold, and all pixels below the threshold would be considered as pixels belonging to the sclera. That threshold is obtained by calculating the biggest group derivative within the range in a histogram of saturation values (between 0 and 0.21 ) corresponding to the image. With the threshold calculated from the original color image in Figure 4.2 (a), Figure 4.2 (b) illustrates an example of extracting the sclera pixels from all other pixels, except for the inherent noise points.

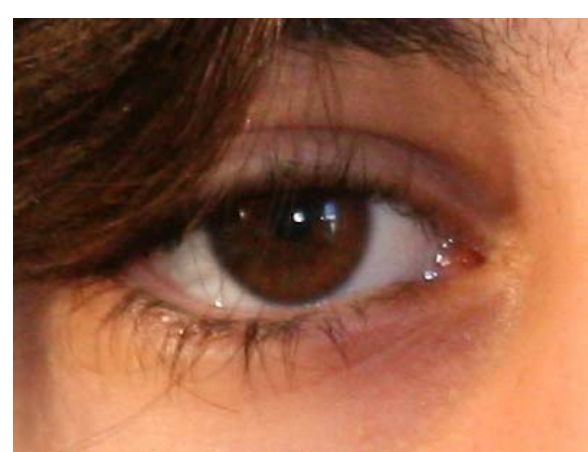

(a) Input eye image

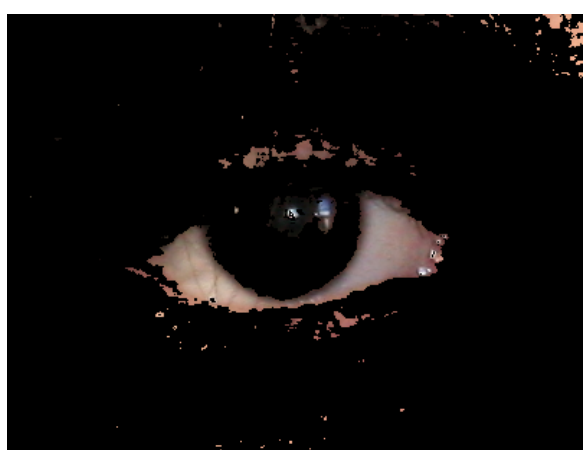

(b) Sclera extraction results

Figure 4.2: Example of sclera detection in the presence of noise pixels

\subsubsection{Determining a target area for the eye}

The purpose of this step is to locate a target area with adaptive size in order to adjust for the different irises of the dataset. After the sclera detection step, it can be observed that some small spots of reflections and bright skin are scattered on the image as part of the sclera extraction step. Thus, more specific sclera areas need to be localized first, to overcome/eliminate the noise points. In opposition to the sclera part, all other spots extracted are generally smaller or slightly darker. The thresholded image, as in 
Figure 4.2 (b), is converted to a grayscale image, and for every pixel with a gray level intensity greater than 0 , its value will be replaced by the average intensity value of a 17 by 17 block which is centered on that pixel. With this operation, the intensity of isolated and relatively smaller noise areas would be degraded significantly. For those pixels whose gray level intensities are greater than 0 , the average intensity is calculated to be an adaptive threshold. With this threshold, a binary map which can clearly specify the sclera area can be generated.

The resulting binary maps, as can be seen in Figure 4.3, can be classified into two categories: double sclera areas as shown in Figure 4.3 (a), and single sclera area as shown in Figure 4.3 (c). In binary maps with double sclera appearing, the iris would certainly be located between them, and the rectangular area can be determined by the extreme coordinates of upper, lower, left and right locations of the two sclera areas, with a small offset proportional to the distance between these extreme points in relation to the image boundaries, in order to ensure inclusion of all the iris. The target rectangle given in Figure 4.3 (b) shows the result for a given image. With a single sclera area, since it is unknown as to which side of the sclera the iris will be located, the nearest distances between the sclera area to the left and right boundaries of the image would be calculated, respectively. The rectangular area would be decided with respect to those distances. The longer the distance is, the more the rectangle would be extended towards the boundary in that direction. As shown in Figure 4.3 (d), the rectangle was mostly extended towards the right side, and as a result the iris part is within the rectangle, which correctly delineates the target area. 


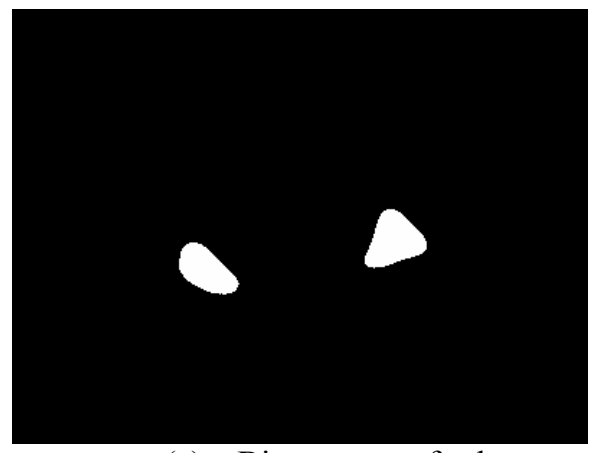

(a) Binary map of sclera

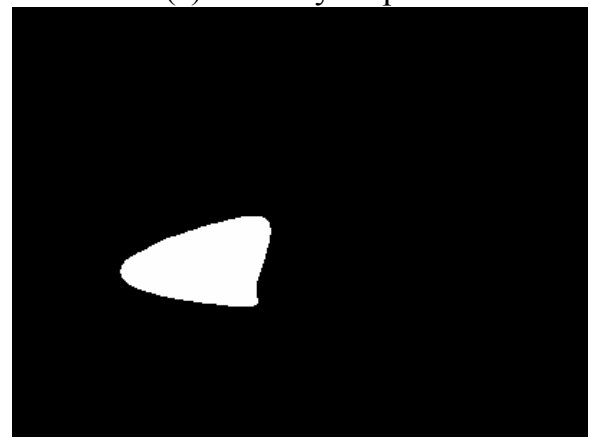

(c) Binary maps of sclera

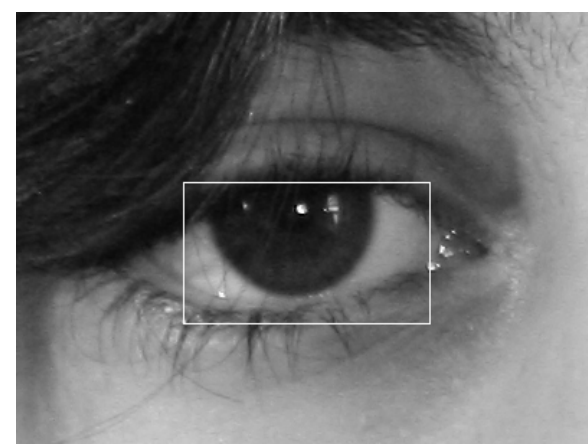

(b) Target area delineation

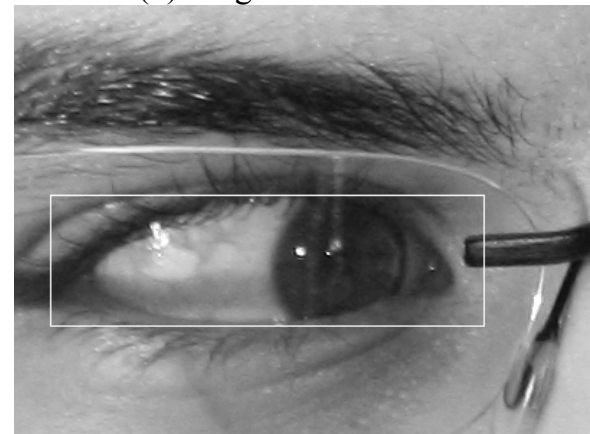

(d) Target area delineation

Figure 4.3: Target selection for eye area based on double and single sclera areas

\subsection{Iris outer boundary detection with a fast circular Hough transform}

The outer boundary of the iris is defined as the boundary between iris and sclera. To detect the outer boundary of noisy iris images from the UBIRIS.v2 database, the circular Hough transform was applied with some modifications to achieve higher accuracy and faster speed. Recall that because the Hough transform is based on a voting scheme, it is quite tolerant to noise (Leavers 1992). 


\subsubsection{Detecting the outer boundary}

To improve the performance of the circular Hough transform, some modifications were made towards both reducing the computational complexity as well as reducing the impact of the inherent noise effects.

To generate the edge map, instead of the traditional four-direction Sobel edge detection (Kanopoulos, Vasanthavada et al. 1988), we only conducted the edge detection horizontally (left to right and right to left), as can be seen in Figure 4.4 (c); these edge points can be compared with those in Figure 4.4 (b), which were generated using all four directions. It is apparent from this comparison, which although, the number of edge points was much reduced; the delineation of the iris is still effective.

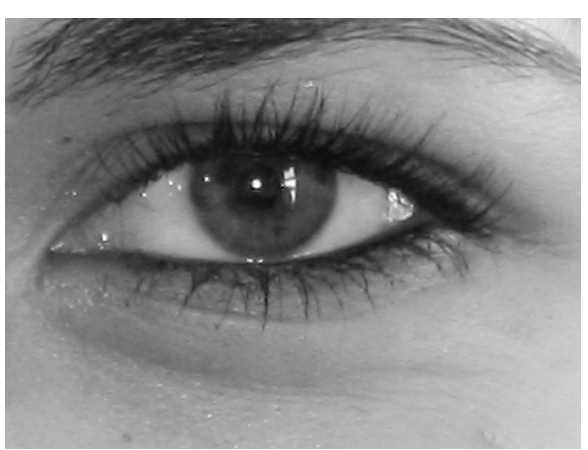

(a) Input image

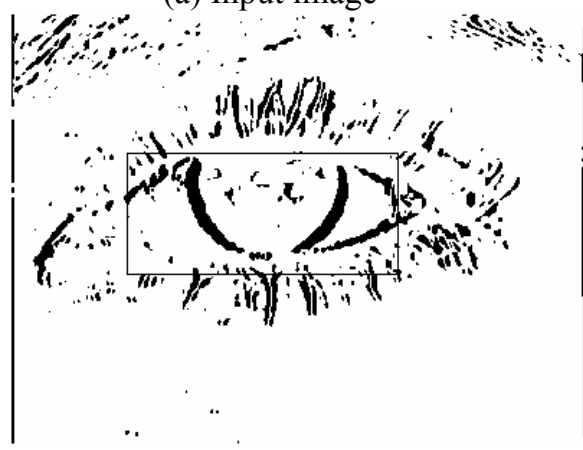

(c) Edge map of 2 directions

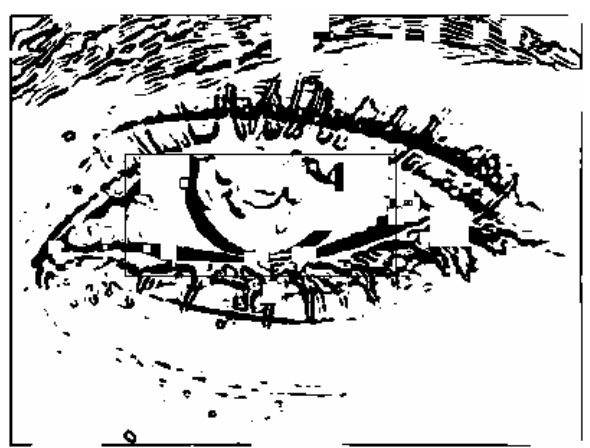

(b) Edge map of 4 directions

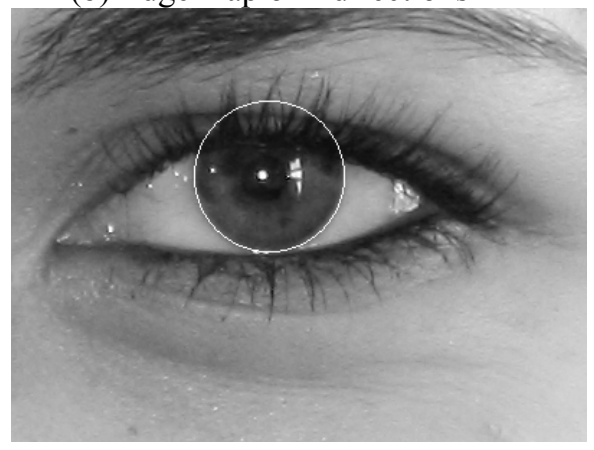

(d) Boundary detection result

Figure 4.4: Example of outer boundary detection using the modified circular Hough transform 
To improve the accuracy and processing speed, after generating the edge map similar to those in studies reported in (Adjouadi, Riley et al. 1995; Adjouadi, Candocia et al. 1996; Chen, Wang et al.), based on the information obtained from the previous step, the circular Hough transform would only be conducted using the edge points in the target rectangle. Also, some precautions are considered. First, the upper and lower limits of the radius can be set with respect to the size of the rectangle. Thus, the upper limit of the radius is set as $1 / 2$ of the rectangle's length, and the lower limit is set as $1 / 4$ of the rectangle's width. Second, neither the center of the resulting circle center nor its boundary can possibly be located on the already defined sclera areas.

\subsubsection{A fast Circular Hough Transform}

Although the circular Hough transform is a powerful algorithm, it also carries with it a heavy computational load, and that refers to the three step iterations burden(Leavers 1992). With the circular Hough transform, each edge point $(x, y)$ in the image space votes for $(a, b, r)$ in the parameter space for each possible circle passing it, where $a, b$ are the coordinates of the circle center position, with $r$ being the radius of the circle. Thus, the votes were cast in a three-direction accumulator for conducting the circular Hough transform. If $O_{1}$ is the computational complexity of calculating votes for a circle with a determined center location and radius, the computational complexity of the circular Hough transform $O_{a}$ is as given by Eq. (2):

$$
O_{a}=\left(a_{\max }-a_{\text {min }}\right) *\left(b_{\text {max }}-b_{\text {min }}\right) *\left(r_{\text {max }}-r_{\text {min }}\right) * O_{1}
$$


Here the step-length was applied to reduce the computational burden of the circular Hough transform, if $C_{a}, C_{b}, C_{r}$ are the step-length for parameters $a, b, c$ respectively, then the computational complexity would be as given by Eq. (3):

$$
O_{b}=\left(a_{\text {max }}-a_{\text {min }}\right) *\left(b_{\text {max }}-b_{\text {min }}\right) *\left(r_{\text {max }}-r_{\text {min }}\right) * O_{1} /\left(C_{a} * C_{b} * C_{r}\right)
$$

In the implementation of the proposed approach, the step lengths are set such that $C_{a}=C_{b}=C_{r}$.

Because of the three step-lengths, a large number of votes will not be counted. To overcome this problem, with a determined circle center and radius, instead of only searching for the edge points located on the circle, all points located on the circular ring surrounding that circle would be counted. For instance, as shown in Figure 4.5, instead of searching for edge points located on the solid circle 1, the search will also include all points within the dashed circles A and B; similarly, all points within dashed circle B and $\mathrm{C}$ would count as votes for the target searching circle given by solid circle 2 .

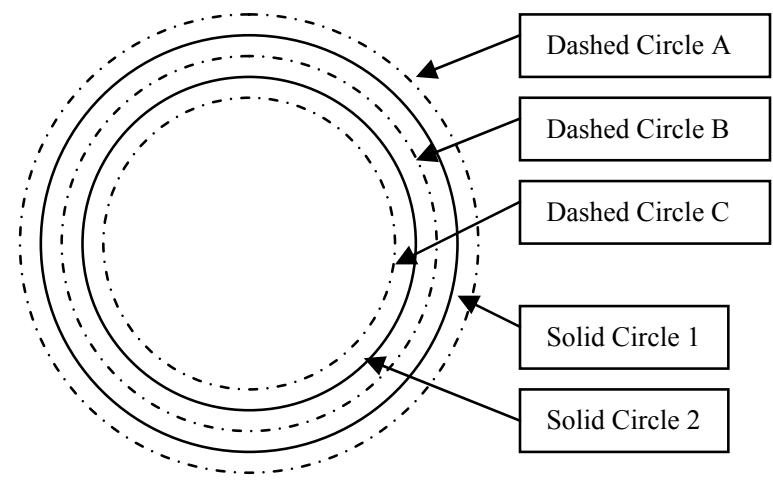

Figure 4.5: Example of searching feature points locating on the circular ring around the target circle 
We also accelerate the search step of all the edge points located on the circular rings. We implemented the algorithm using dynamic programming (Sacco 1987; Robinett 2005). Initially, the distance between every pixel point on the image and the image center point would be calculated, all those distances would be stored in the table, and each distance would refer to a list of relative locations which have that certain distance from the center location of the image frame. When searching for the edge points on the circle, the neighboring distances would refer to a set of relative locations in the look-up table, and all edge points located on the corresponding locations in the image would be counted. Because the look-up table was generated just after executing the proposed approach for a single image, and since the size of all input images is $400 \times 300$, there is no need to calculate distances while performing the Hough transform for each image, and the computational burden was consequently alleviated significantly.

Finally, to obtain more consistent results, instead of just choosing the peak value of votes in the parameter space, a range of highest values (for this research, all the parameters have a value within $5 \%$ of the peek value votes) are used instead; by examining their neighboring parameters which are bypassed because of using step-length, more precise results can now be accomplished.

To demonstrate the performance of the proposed method, the step-length is set to be between 1 and 5, and the processing time is examined based on all 500 eye images from the UBIRIS.v2 training database. The results are as shown in Figure 4.6, where the step-lengths for $\mathrm{C} 1$ through $\mathrm{C} 5$ are 1 through 5, respectively. 


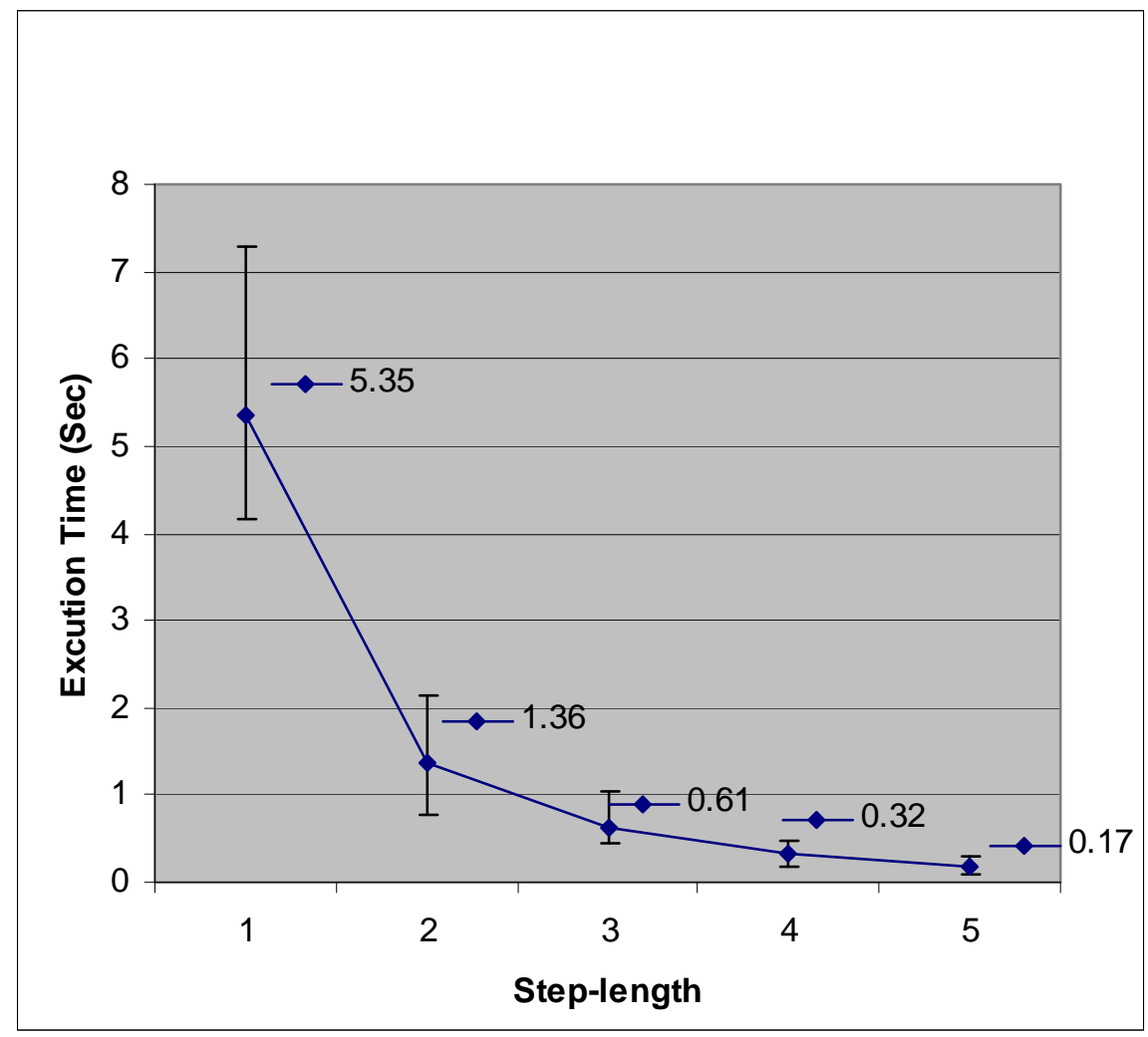

Figure 4.6: Average processing time for circular Hough transform using 500 images from UBIRIS.v2 training database

This modified circular Hough transform decreases the execution time of the proposed approach significantly. The average processing time using the circular Hough transform which applies a step-length of 1 is calculated as $6.77 \mathrm{~s}$ per image, and the error rate of such an approach is estimated at 0.0200215 in the E1 measure provided by the Committee of NICE.I. With the proposed modified circular Hough transform, the average execution time is decreased to $0.83 \mathrm{~s}$ and the E1 error rate is 0.0200823 now. With the proposed modified Hough transform, the execution time is significantly decreased by a factor of 8.2 times, while the accuracy is only degraded by $0.303 \%$, which is considered negligible. Execution time and E1 error rates for both cases are examined by processing the provided 500 iris images from the UBIRIS.v2 database using a laptop computer with 
1.86GHz CPU (Intel T1350 solo) and 2GB of RAM. The executable program we submitted to the NICE.I committee is the fast version, which used the modified circular Hough transform with a step-length of 5.

\subsection{Detecting the boundary for upper and lower eyelids}

In unconstrained iris acquisition scenarios, as the subject is not required to comply with a demanding level of cooperation, it is observed that eyelid occultation can occur. The linear Hough transform can be applied to the edge map of the eye image to detect the eyelids. However, with this algorithm, the key problem for dealing with noisy eye images is that the obtained edge maps are usually non-ideal. As shown in Figure 4.7 (a), most edge points appearing on the map are undesired, caused by eyelids, reflections inside the iris, and the texture of the iris patterns, among others. To overcome this problem, an improved strategy for eyelid detection is proposed. Because the slopes of the upper and lower eyelids are not steep in most cases, the proposed approach starts by applying edge detection in only the vertical direction. The generated edge map will have an emphasis on the desired eyelids edge points. Figure 4.7 (a) is one such example.

The proposed approach has shown good performance for processing noisy iris images from the UBIRIS.v2 dataset. Figure 4.7 illustrates an example for eyelid boundary detection. This is by all means perfect as can be seen from Figure 4.7 (d) where a tiny portion of the eyelid at the intersect of the Hough line for the lower eyelid and the circle containing the iris has been assigned to the delineated iris region. But these are tolerable mistakes for the iris recognition process. 


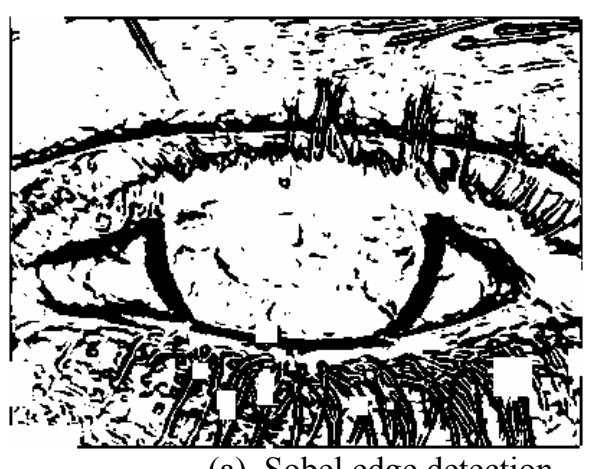

(a) Sobel edge detection

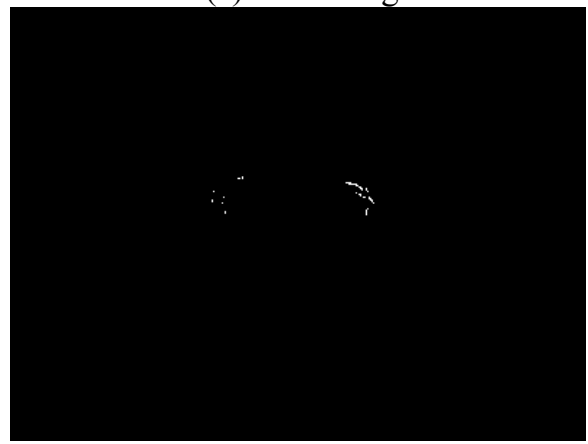

(b) Upper eyelid boundary

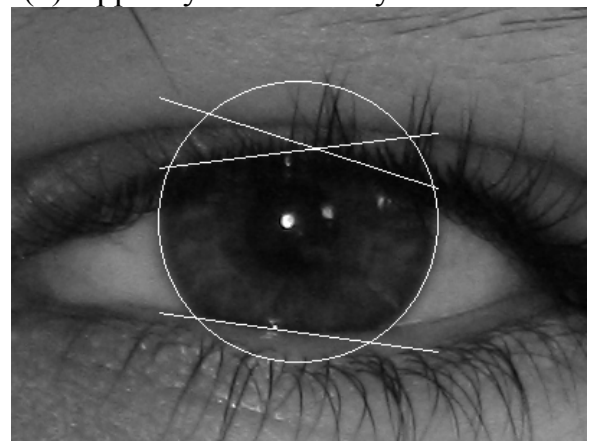

(c) Left eyelid boundary

(d) Eyelid boundary detection

Figure 4.7: Example for detecting upper and lower eyelids

To distinguish the points which are edges between iris and eyelids, a patch of area is selected to calculate the average gray intensity $I_{A}$ of the iris, as shown in Figure 4.8. A square patch just below the pupil is chosen, since this area is experimentally determined to be the one most unlikely to be affected by reflections or overlap with eyelids. Because iris sizes vary significantly within the given UBIRIS.v2 dataset, the size of the patch is set to be adaptive in relation to the size of the iris. For this research we set the length of the square to be $R_{\text {outer }} / 10$, with $R_{\text {outer }}$ being the radius of the outer iris boundary. The upper boundary of the patch is located $R_{\text {outer }} / 8$ below the pupil's lower boundary. 


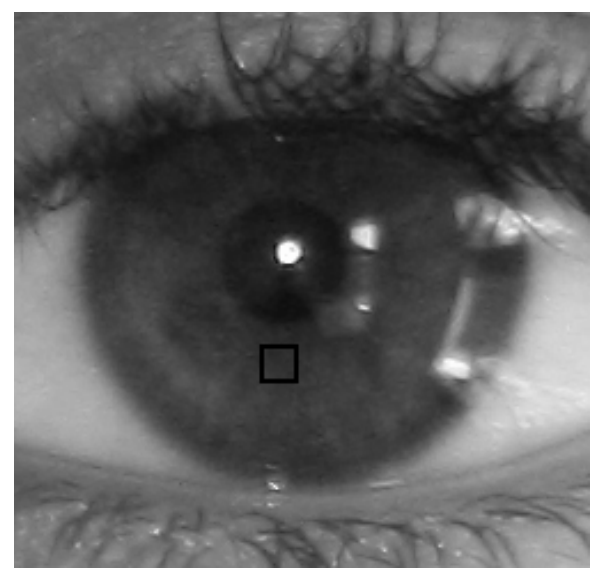

Figure 4.8: Example of obtaining average gray intensity

With the outer boundary circle obtained as in Figure 4.7 (d), rays are traced starting from the center of the circle and toward every point on the upper or lower arc of the circle (central angle $\theta=120^{\circ}$ ). For each ray, if an edge point was found, $M$ points beyond this point in the ray's direction would be verified using the average gray scale $I_{A}$ to see if it is within a range of $I_{A} \pm 10 \%$; if $N$ of $M$ points can be considered as points on the iris, the found edge point would be ignored in order to eliminate potential smaller reflection regions and iris regions with textural properties, and the search would go on until reaching the circle boundary. For this particular implementation, $M=R_{\text {outer }} / 6$ and $N=R_{\text {outer }} / 10$. The example given in Figure 4.7 (d) shows the verified edge points for the upper eyelid. After the verified edge map was generated, the linear Hough transform was then used. Since most of the undesired edge points were removed before this step, the results of the linear Hough transform are more accurate and tolerant to noise.

For some cases, it could be more feasible to describe the eyelid with multiple lines. In the proposed approach however, after the first line was detected, the edge points 
on that line were moved and so were all edge points located within 5 pixels above and below that same line. The linear Hough transform is then applied again, and if there are enough votes, another line would be determined, and so on. Then point removal and Hough transform would be applied again. This process would continue until the peak value of the linear Hough transform is below a threshold, which in this case was set as $R_{\text {outer }} / 4$. Referring back to Figure 4.7, Figure 4.7 (b) shows the detected points which are on the edge of iris and upper eyelid, Figure 4.7 (c) shows the remaining points after the first linear Hough transform for the upper eyelid, and Figure 4.7 (d) shows the final results of boundary detection for upper and lower eyelids. Two other results from the UBIRIS.v2 database are shown in Figure 4.9 as illustrative examples.
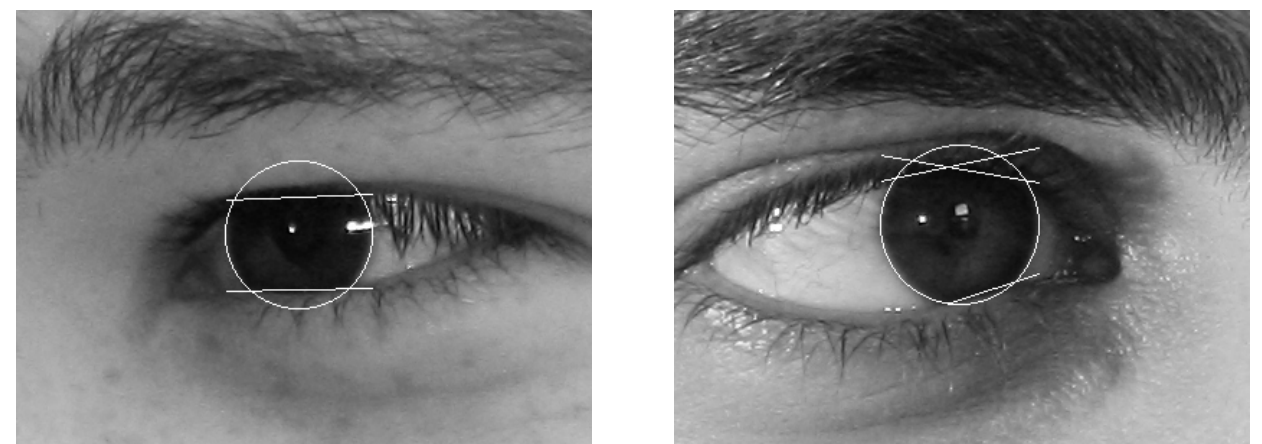

Figure 4.9: Results of eyelid boundary detection using two different subjects

\subsection{Circle correction and non-circle boundary detection}

The error caused by defining the boundary as a circle is especially crucial for less constrained iris recognition, because of ubiquitous off-angle effects during noncooperative iris acquisition. For this reason, a strategy was developed to define the noncircle boundary and correct the inaccurate result obtained from the circular Hough transform. 
Figure 4.10 (a) is one example of outer boundary detection error caused by a noncircular iris boundary. As can be seen, the outer boundary cannot be correctly detected. The proposed verification and correction method is based on three steps; (1) the circle center verification, (2) multiple circle (or arc) searching, and (3) multiple arcs and lines connection.
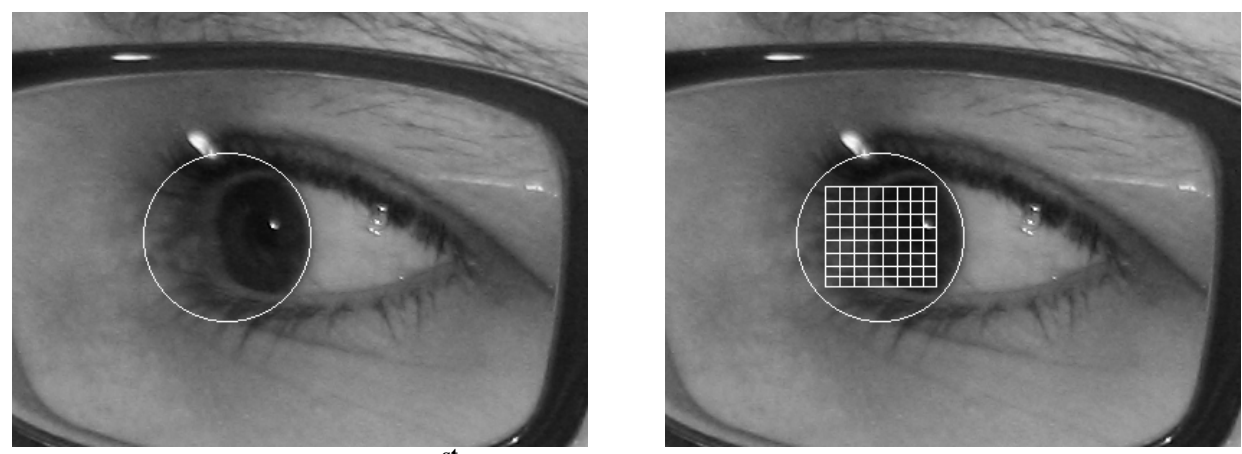

(a) Results of the $1^{\text {st }}$ Hough transform (b) Grid region overlay

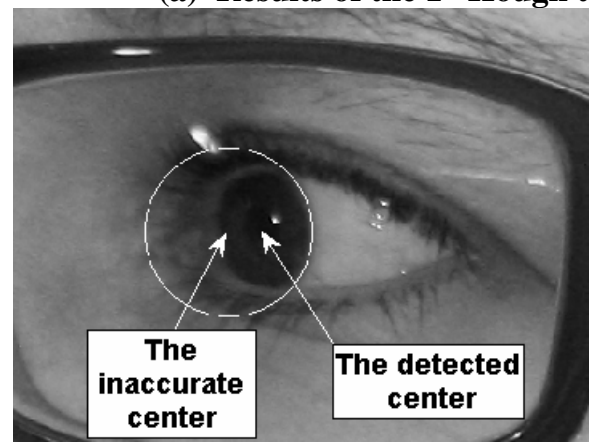

(c) Original and detected new center locations

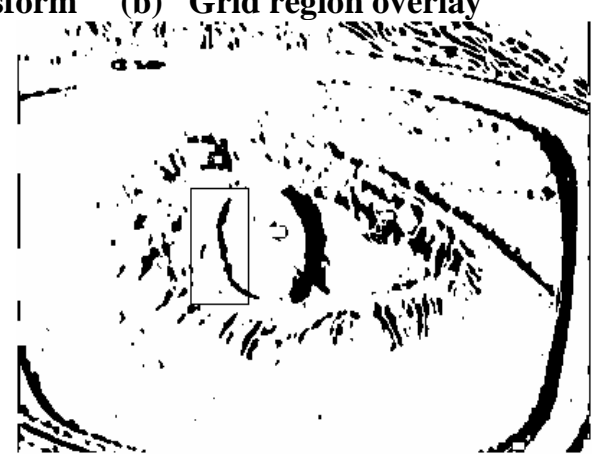
target region

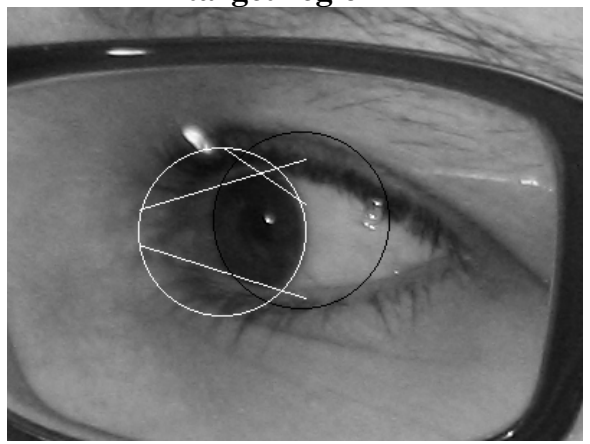

(d)

Selected

(e) Hough circle (in black) of target area

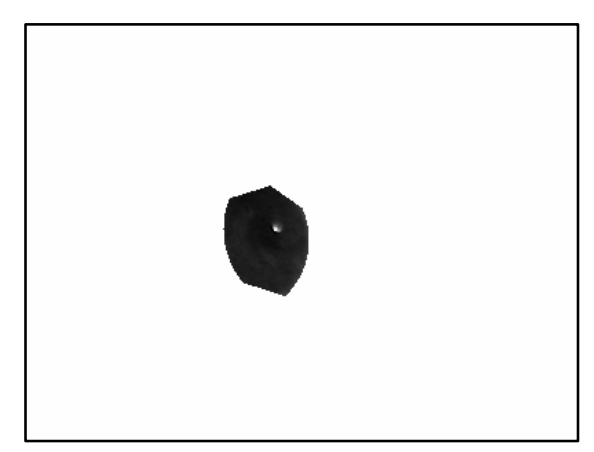

(f) Final iris detection results

Figure 4.10: Defining the noncircular iris boundary- the intersection of the two circles with the detected eyelids lines is the accrual iris 
There is a need to find the correct center of the iris to verify the original circle center found from the first Hough circle transform. Shown in Figure 4.10 (b) is a square grid with an adaptive size inside the outer iris boundary. The center of the grid which yields the lowest average gray intensity would be selected as the correct outer iris boundary center. For the purpose of this research, if the Euclidean difference between the original center and the newly detected one is greater than $R_{\text {outer }} / 4$, the original outer boundary would be assumed as inaccurate. Then a target area would be selected for conducting Hough circular transform again. Based on the experimental study, the desired iris boundary is usually inside the Hough circle. The reason for this is that the edge points outside the iris usually vote for larger circles and for an off-angle iris which is usually smaller than its actual size. Thus, the target area whose center is at $\left(x_{t}, y_{t}\right)$ as shown in Figure 4.11 was expected to be the region between the real iris center $\left(x_{r}, y_{r}\right)$ and the arc on the opposite side of the original circle.

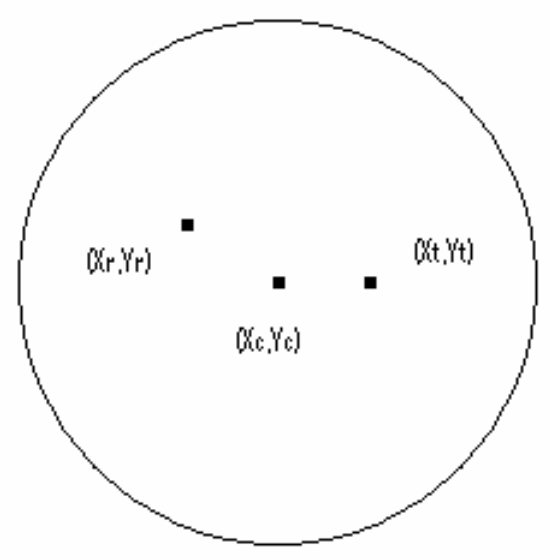

Figure 4.11: Relations between the real iris center, original circle center and center for target rectangle 
In reference to Figure 4.11, the center of the target rectangle is $\left(x_{t}, y_{t}\right)$, and the original circle center is $\left(x_{c}, y_{c}\right)$, here we have:

$$
y_{t}=y_{c}, \text { and } x_{t}=x_{c}-\left(x_{r}-x_{c}\right) \text { or } x_{t}=2 x_{c}-x_{r}
$$

Another Hough transform is conducted on the edge points inside the target rectangle. Because the goal is to detect a circle which is usually partially located on the target region, different weights are set to the votes generated inside and outside of the target region to obtain better results. In our experiments, we set the weight of the vote as 1 for the outside region and as 7 for the inside region.

Since the Hough transform is based on voting, to avoid uncertain results, an adaptive voting count threshold is set such that if the highest voting count is below this threshold, the circle will not be generated. This threshold is set as $T=M * 35 \%$, where $M$ is the total count of edge points in the target rectangle. If the peak value $N<M$, the circle will not be considered. The black circle shown earlier in Figure 4.10 (e) is one outcome example of this step.

The intersection of two circles can be used to describe the noncircular iris. The upper and lower eyelids lines are also used here to remove unexpected eyelids and eyelashes. Thus, as can be seen from Figure 4.10 (f), the final result of the detected boundary consists of multiple arcs and lines, and would be more accurate for describing noncircular iris boundaries. Other examples provided for comparative purposes are shown in Figure 4.12. 


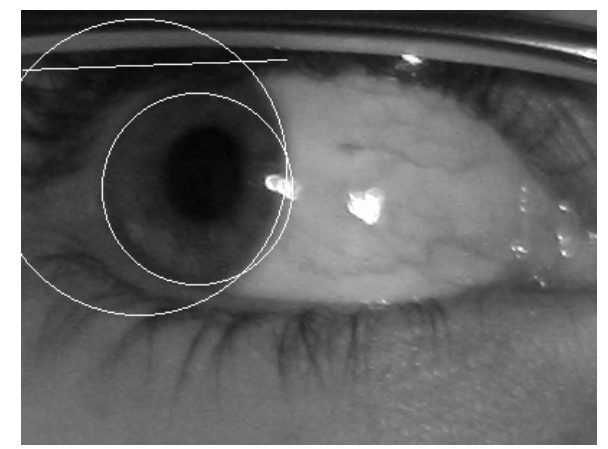

(a) Circle correction: case 1

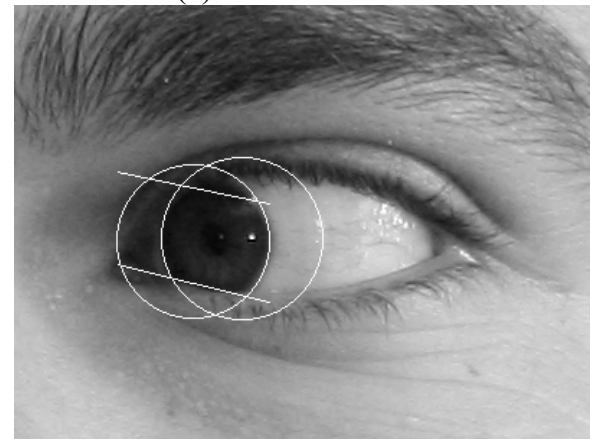

(c) Delineating iris boundary

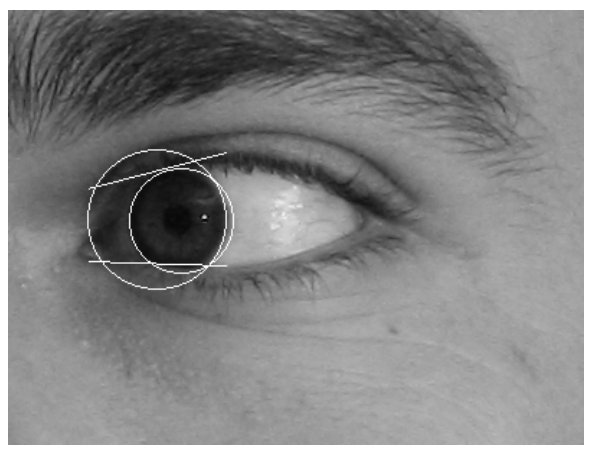

(b) Circle correction: case 2

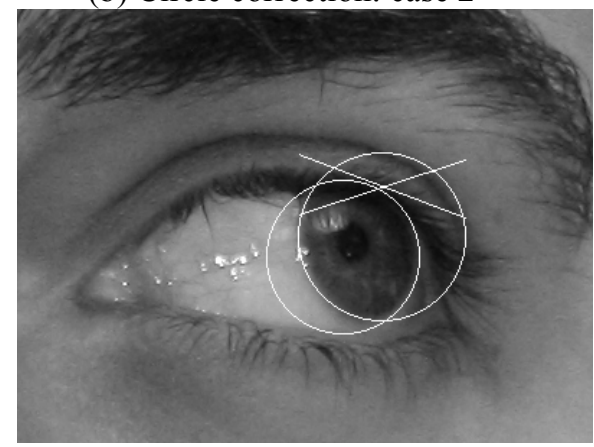

(d) Delineating iris boundary

Figure 4.12: Result examples of proposed circle verification and correction strategy for delineating noncircular iris boundaries

Inaccuracies introduced by the circular Hough transform can thus be resolved with the proposed method. As shown in Figure 4.12, the larger circles shown in (a) and (b) are inaccurate circular Hough transform results, and the smaller circles in both cases are the newly detected ones through the proposed method. As can be seen, the intersections are much more accurate. Figure 4.12(c) and Figure 4.12(d) are two other examples with different iris sizes and different eye gazes. The left circle shown in Figure 4.12(c) and the right circle shown in Figure 4.12 (d) are the original Hough transform results, which, as can be seen, are slightly off from the expected result. 


\subsection{Pupil, eyelash detection and results reliability verification}

Because the eye images from the UBIRIS.v2 dataset were captured under visible wavelength, one of the major differences with those images taken under NIR wavelength is that the intensity contrast of iris and pupil can be very low, especially for heavily pigmented (dark) irises, such as in Figure 4.13.(a). Thus, pupil removal is left for this step to be performed; with only iris and pupil, the contrast enhancement method would yield better performance (Verdenet, Cardot et al. 1981).
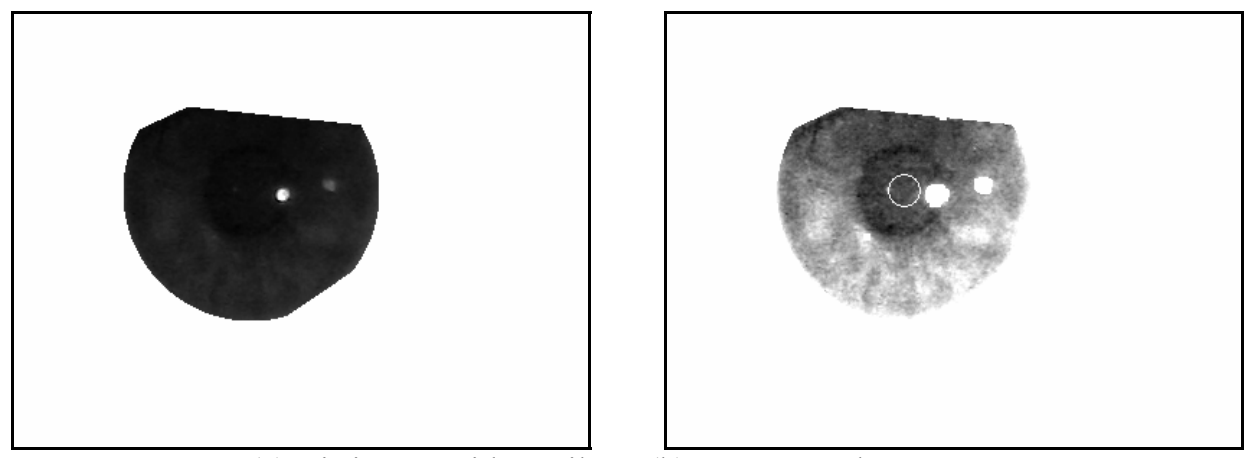

(a) Iris image with pupil

(b) Contrast enhancement

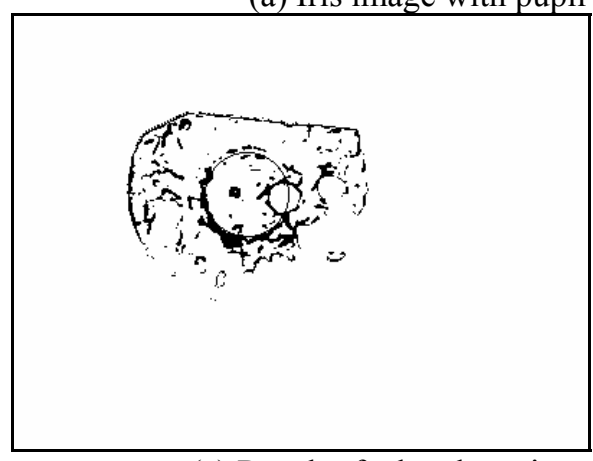

(c) Result of edge detection

(d) Pupil removal result

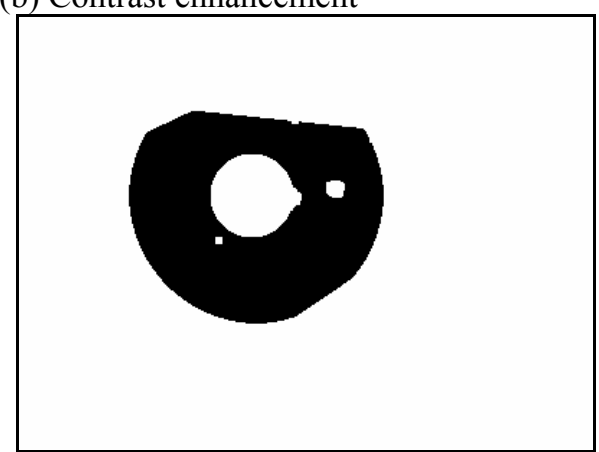

Figure 4.13: Process for pupil detection and removal

As shown in Figure 4.13, image (a) is the outcome from the previous step (outer iris and upper and lower eyelid boundary delineation). We used an empirical intensity threshold of 150 to detect the reflections, and expanded every reflection point by a $3 * 3$ 
mask to ensure its total removal. Then, histogram equalization was applied to get the high-contrast image, as shown in Figure 4.13 (b). Sobel edge detection was used to get the edge map (c), and then the circular Hough transform determined the pupil boundary. Caution is taken such that the pupil center should be located within the small white circle shown in Figure 4.13 (b) with a radius of $R_{\text {outer }} / 10$, and its center can be considered as the outer iris center. The radius of the pupil boundary is set to be from $3 R_{\text {outer }} / 20$ as a lower limit and to $11 R_{\text {outer }} / 20$ as the upper limit. Figure 4.13 (d) shows the result for this particular example. To detect the eyelashes, we set an adaptive empirical threshold to be $0.45 * I_{A}$, where $I_{A}$ is the obtained average iris color as described in Section 4.4. The eyelash removal would be performed within the top $R_{\text {outer }} / 3$ part of iris. After the pupil and eyelash removal, the result of the segmentation approach would be generated.

To ensure that the falsely segmented results would not pass to the next step of iris recognition, a reliability verification procedure was applied to minimize false positives. For the UBIRIS.v2 dataset, if the segmented iris is too big $\left(R_{\text {outer }}>120\right)$, too small ( $\left.R_{\text {outer }}<20\right)$, too bright $\left(I_{A}>90\right)$, or if the average intensity of the pupil is brighter than the average iris intensity, the segmented result would be rejected. 


\subsection{Result and evaluation}

Some of the good results are shown in Figure 4.14. The green and red parts on the iris area are the non-matched errors as determined by the NICE.I committee through an unbiased evaluation.
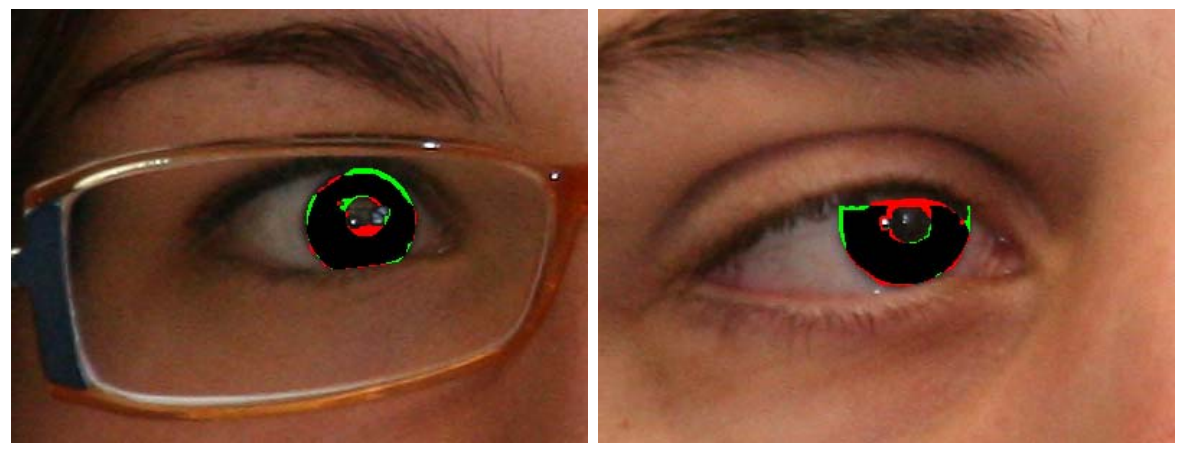

Figure 4.14: Examples yielding good results

As shown in Figure 4.14, almost all the function modules of the proposed approach worked very well. Some errors occurred on the outer iris boundary, because a single circle is used to define the boundary, but those errors are found to be relatively small. Through the same unbiased evaluation, some of the worst results were caused by sclera detection errors, as shown in Figure 4.15(a). For this image, the sclera area was found to be the bright part of the upper eyelid; thus, the target rectangle was falsely set to be out of the iris region. Figure 4.15 (b) shows that with an incorrect searching target area, unpredictable results would be obtained from the circular Hough transform; the black circle on the top-right of the image is the outcome of iris boundary detection which was conducted in the non-iris region. In the results given in Figure 4.15 (c), the green area refers to false positive errors, and the red area refers to false negative errors. 


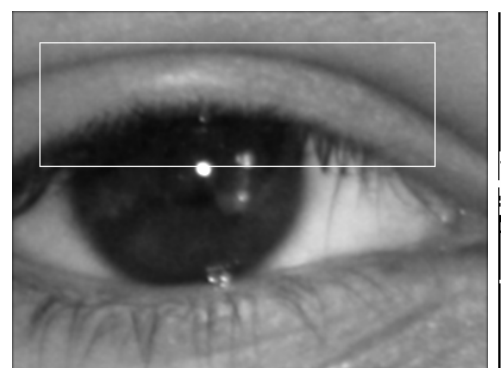

(a) Eye area selection

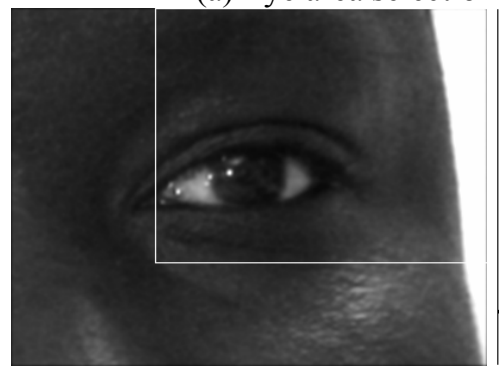

(d) Eye area selection

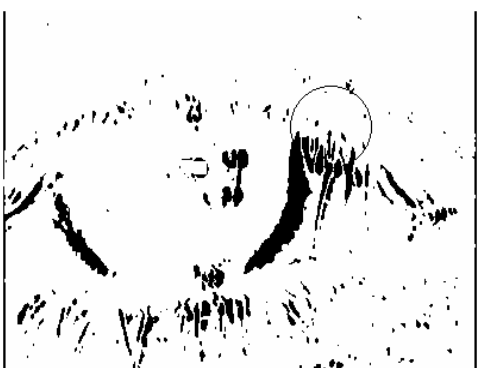

(b) iris boundary detection

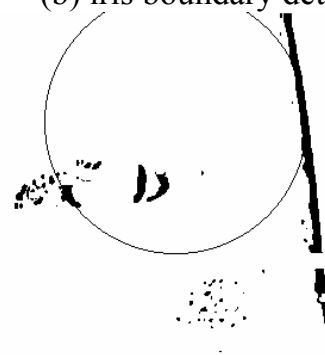

(e) iris boundary detection

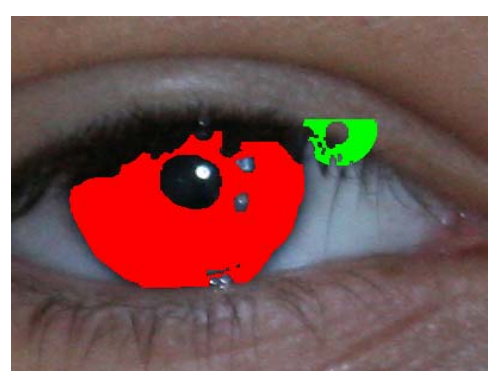

(c) error

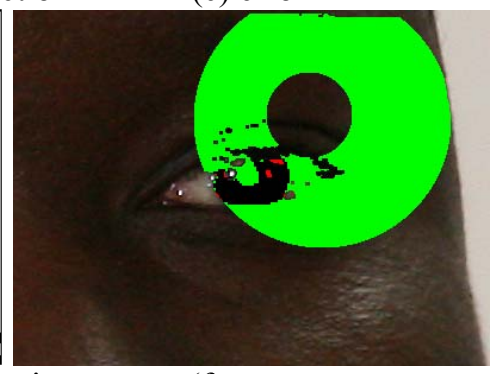

(f) error

Figure 4.15: Examples yielding faulty or undesired results

As for the example given in Figure 4.15 (d), because the white wall behind the subject is detected to be sclera, the target rectangle was set to be too large for the actual iris region, and the upper limit radius of the target circle was set to be too large (recall that the upper limit of the radius is set to be half of the rectangle length in the proposed approach). As shown in Figure 4.15 (e), in that search area, a very large circle can get more votes than the actual boundary circle. Thus, as shown in Figure 4.15 (f), incorrect results are obtained, with the red (false negative) being small because the incorrect result region incidentally covers the real iris area.

The UBIRIS.v2 dataset includes rotated iris images, and some of which with angles that are more than 30 degrees (relative to the horizontal). Through our experiments on the provided training dataset, it has been noticed that the rotation effects may impact the step of locating the target iris area which is introduced in Section 4.2.2, although 
those impacts are mostly overcome. There are 17 images with the rotations greater than 30 degrees relative to horizontal, and only two cases lead to inaccurate iris outer boundary detection results.

As the target rectangle area is set to be a horizontal rectangle, for those rotated iris images, the determined target rectangle may cover a partial iris area instead of the whole iris area. Because the voting-based circular Hough transform would be used to search for the outer iris boundary, a partial circle within the target area can still determine the outer boundary. Furthermore, the target rectangle area is extended with respect to the location and size of the sclera area; and for most of the rotated iris images, an adequate portion of the iris would still be covered, providing desirable outer boundary detection results even under these extreme conditions.

As shown in Figure 4.16 (b), although the target rectangle does not cover the whole iris boundary, the rectangle is extended downwards to cover more of the boundary edge points. The white circle drawn on Figure 4.16 (c) shows the accurate result of the outer boundary detection. An inaccurate outer boundary detection caused by rotation effect is also given in Figure 4.16 (d) and (f). As can be seen in Figure 4.16 (e), since the detected sclera area is located just below the middle of the image, the target is slightly extended upwards. The target rectangle covers relatively a small portion of the iris area; with other noise effects on the edge map such as eyelashes and eyelids. The inaccurate outer boundary detection that resulted, as reflected by the white circle drawn on Figure 4.16 (f), is due to inaccurate positioning of the rectangle. 


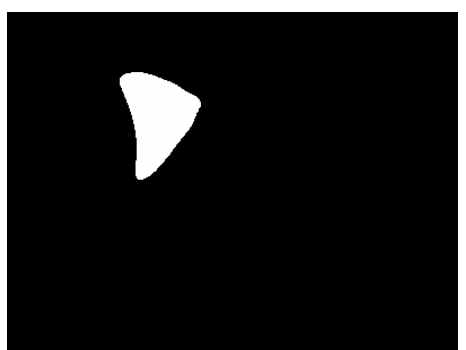

(a) Binary map

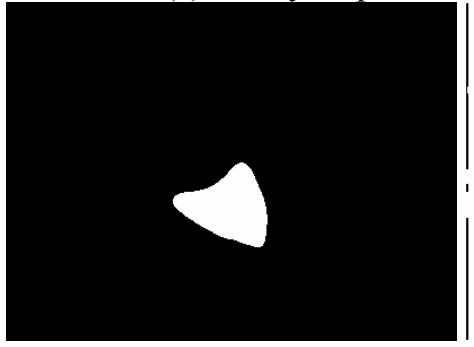

(d) Binary map

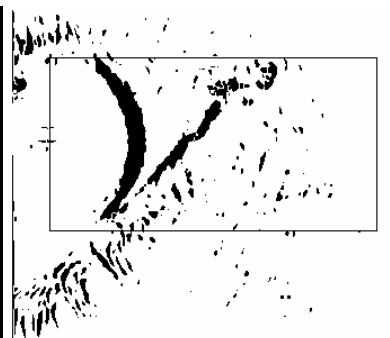

(b) Target area on edge map

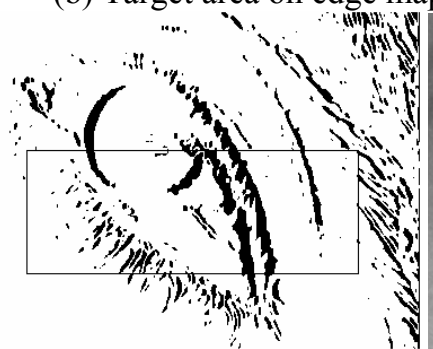

(e) Target area on edge map

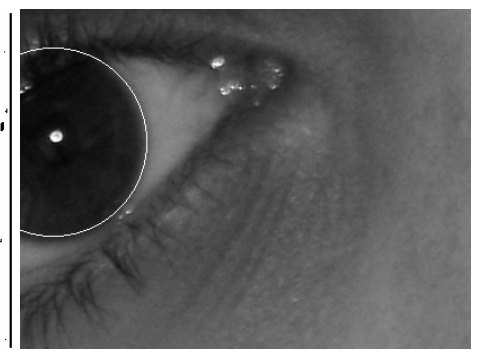

(c) Accurate result

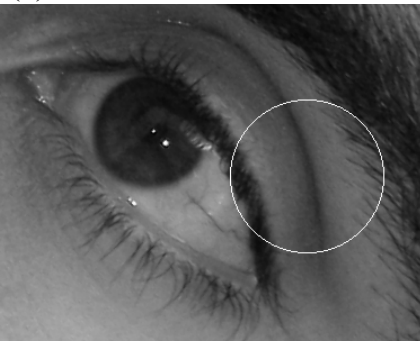

(f) Inaccurate result

Figure 4.16: Examples of detecting the outer iris boundary of rotated iris images

In retrospect, through the unbiased evaluation from NICE.I committee, the results of the proposed approach yielded error measures of E1=0.029 and E2 $=0.163$ (where False Positive rate $=0.018$ and False Negative rate $=0.307) . E 1$ and E2 are defined in detail in the NICE.I Evaluation webpage (http://nice1.di.ubi.pt/evaluation.htm).

Just as important, the processing speed of our approach is fast. For the 500 images from the UBIRIS.v2 training dataset, the average processing time is $0.83 \mathrm{~s}$ per image. This result is obtained using a laptop computer with $1.86 \mathrm{GHz} \mathrm{CPU}$ (Intel T1350 solo) and $2 \mathrm{~GB}$ of RAM. The proposed approach was implemented using $\mathrm{C}++$ programming language. 


\section{A Robust Segmentation Approach to Iris Recognition Based on NIR Video}

\subsection{Introduction}

To overcome the constraints imposed on users in the process of taking iris images as indicated in Chapter III, significant efforts have been devoted to the design of different image acquisition setups that can accommodate subjects on the move. As an unconstrained iris recognition approach, video based iris recognition is designed to provide convenience and time efficiency to the subjects during what becomes a latent image acquisition process(Matey, Broussard et al. 2010). These videos of moving subjects at a distance do however introduce unexpected noise effects which can impact the accuracy of the iris recognition process (Hollingsworth, Peters et al. 2009).

To begin addressing this type of less-constrained image acquisition process, this chapter presents a new approach at delineating the iris region through a novel segmentation approach as an essential step for the eventual video based iris recognition(Chen, Wang et al. 2008). The proposed approach consists of two steps. The first step is video frame selection which is to obtain qualified frames from NIR video. The subjects' eye images were extracted based on the featured reflection spots generated by the specified video camera. The second step is iris segmentation which is intended to isolate the iris region from the eye image. Since iris images obtained from NIR video may suffer from different kinds of noise effects, a new strategy is proposed for iris segmentation to include noisy eye images. As a consequence, the proposed iris 
segmentation strategy would not only separate the iris part from the sclera and pupil, but is also able to identify the extraneous overlapping parts caused by eyelids, eyelashes, and reflection spots.

\subsection{Background and Motivation}

This main idea at this juncture is to develop a robust segmentation approach towards video based iris recognition that can deal with various noise efforts caused by video taking at a distance and of moving subjects.

J.R. Matey, O. Naroditsky and other colleagues introduced an iris recognition system called Iris on the Move (IOM) (Matey, Naroditsky et al. 2006), which was based on Near Infrared (NIR) video taken at a distance as it targets moving subjects. The system is claimed to be able to perform iris recognition on subjects walking at a normal pace estimated at $1 \mathrm{~m} / \mathrm{s}$.

For conducting the iris verification or identification, the NIR videos of subjects' faces were taken with a resolution of $2048 \times 2048$. The iris images acquired from the videos are different from images taken using traditional acquisition methods. With the traditional methods, the pixels across iris are usually more than 200 (Daugman 2001; Ma, Tan et al. 2003), but eye images from the videos only offer about 120 pixels across the iris part, which can only exacerbate an already complex problem. As can be seen in Figure 5.1, the eye images in Figure 5.1.c and Figure 5.1.d are form the CASIA database (Phillips, Bowyer et al. 2007), which are acquired from still iris acquisition system, and

can offer about 240 pixels across the iris region. On the other hand, Figure 5.1.a and 
Figure 5.1.b are eye images extracted from the IOM videos, with only about 120 pixels across that can be counted across the iris region.

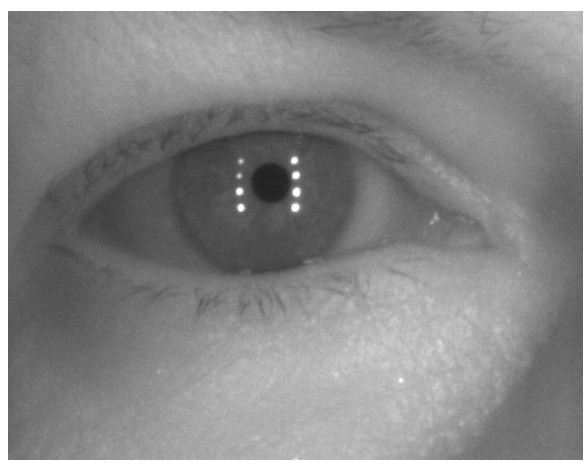

(a)

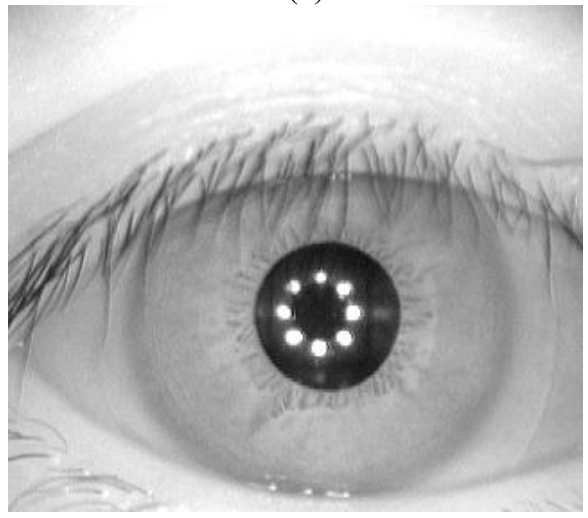

(c)

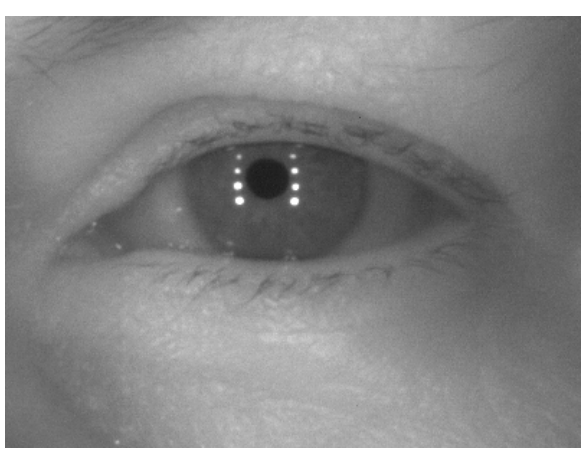

(b)

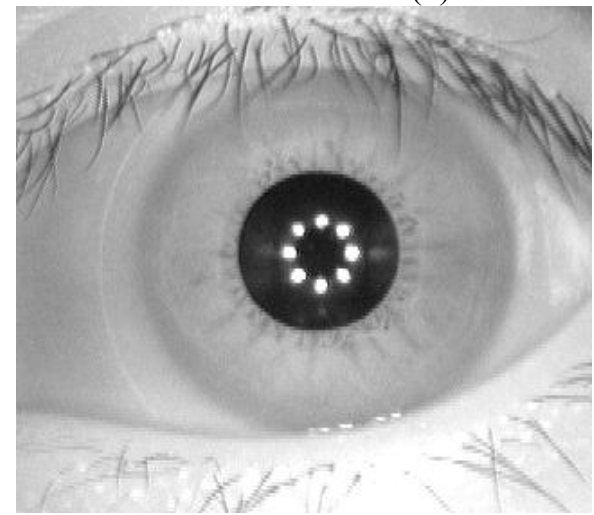

(d)

Figure 5.1: Eye images acquired from IOM system and traditional iris acquisition method: (a), (b) eye images extracted from the IOM system; (c), (d) eye images from CASIA database (version 3 ) using the traditional method.

Because the video of subjects were taken at some distance, J.R. Matey et al. have shown that frames taken of a moving subject walking through a view field which is about $12 \mathrm{~cm}$ in depth yield the perfect focus distance which can later on be used for iris verification (Matey, Naroditsky et al. 2006). While conducting iris verification with the IOM system, with the video yielding 15 frames/s and with the assumption that the subject is moving at a speed of $1 \mathrm{~m} / \mathrm{s}$, there are no more than 2 frames that can be captured within 
that $12 \mathrm{~cm}$ depth of field. Consequently, for subjects on the move, iris images extracted from such limited frames may suffer from various kinds of noisy effects caused by off angle, motion blur, occultation caused eyelid or eyelashes, and unexpected reflections. Some examples of noisy iris image obtained from IOM system are shown in Figure 5.2.

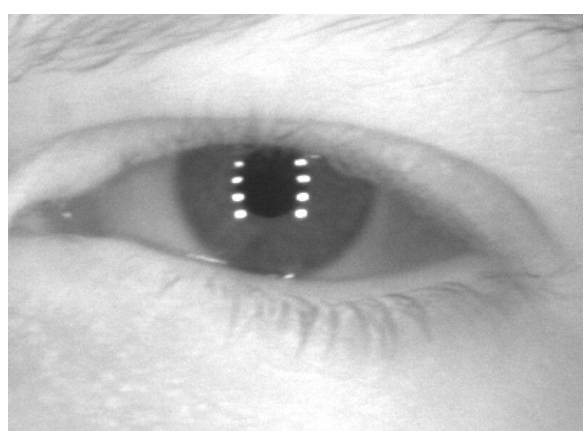

(a)

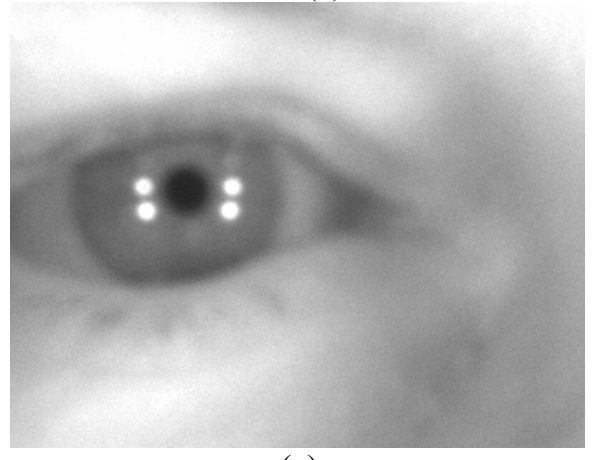

(c)

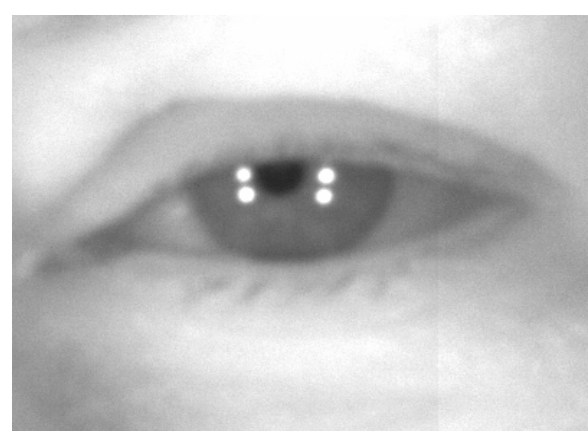

(b)

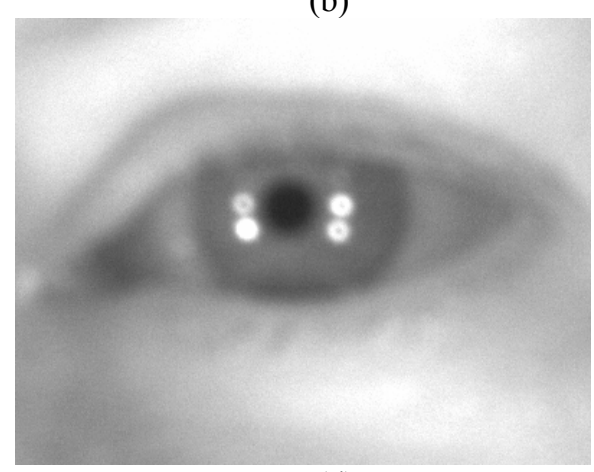

(d)

Figure 5.2: Noisy eye images acquired from MBGC database: (a), (b) eye images with eyelids occultation; (c), (d) eye images with motion blurs

Thus, a good segmentation approach which can extract most useful iris information from the video would be crucial for the performance of video-based iris recognition systems.

For the work presented in this chapter, MBGC database provided by National Institute of Standards and Technology (NIST) is used for experimental evaluation. Also, 
the NIR-face-video sequences used from the database are those that were captured by the IOM system supplied by Sarnoff.

\subsection{Eye Images Extraction and Pupil Localization}

\subsubsection{Eye images extraction from video frames}

The NIR videos of MBGC database have a resolution of $2048 \times 2048$. All the videos that were captured towards were of the subjects' upper bodies while they were walking towards the video camera system. And the eyes of subjects may appear at different locations in the acquired video frames. Thus a necessary first step would be to isolate these eye areas in the video.

Because of the specified NIR illumination system of IOM system, there are 4 pairs of reflection spots that appear at the corneal of each eye. These reflection spots are located in 2 parallel lines which are known to have a horizontal interval of 35 to 50 pixels between them in the frames that are acquired at the aforementioned preset distance.

Our method to separate the eye images from the video frames is based on this particular configuration of the reflection spots. Since the subject is most likely to be walking when the video is taken, it was determined that the 8 reflection spots which usually appear in one eye, do not necessarily appear for both eyes simultaneously. An intensity threshold of 180 is used to extract these spots, and for every detected spot, if there is another spot that can be found within a certain horizontal distance, a pair of reflection spots is declared to have been identified. If there is a second pair of spots that can be found at several pixels below the detected pair, that area would be declared the 
eye part of the subject within a given frame. Figure 5.3 shows an example of extracting eye images from a frame. Eye images for subject's right and left eyes were extracted with a size of $400 \times 300$ pixels.

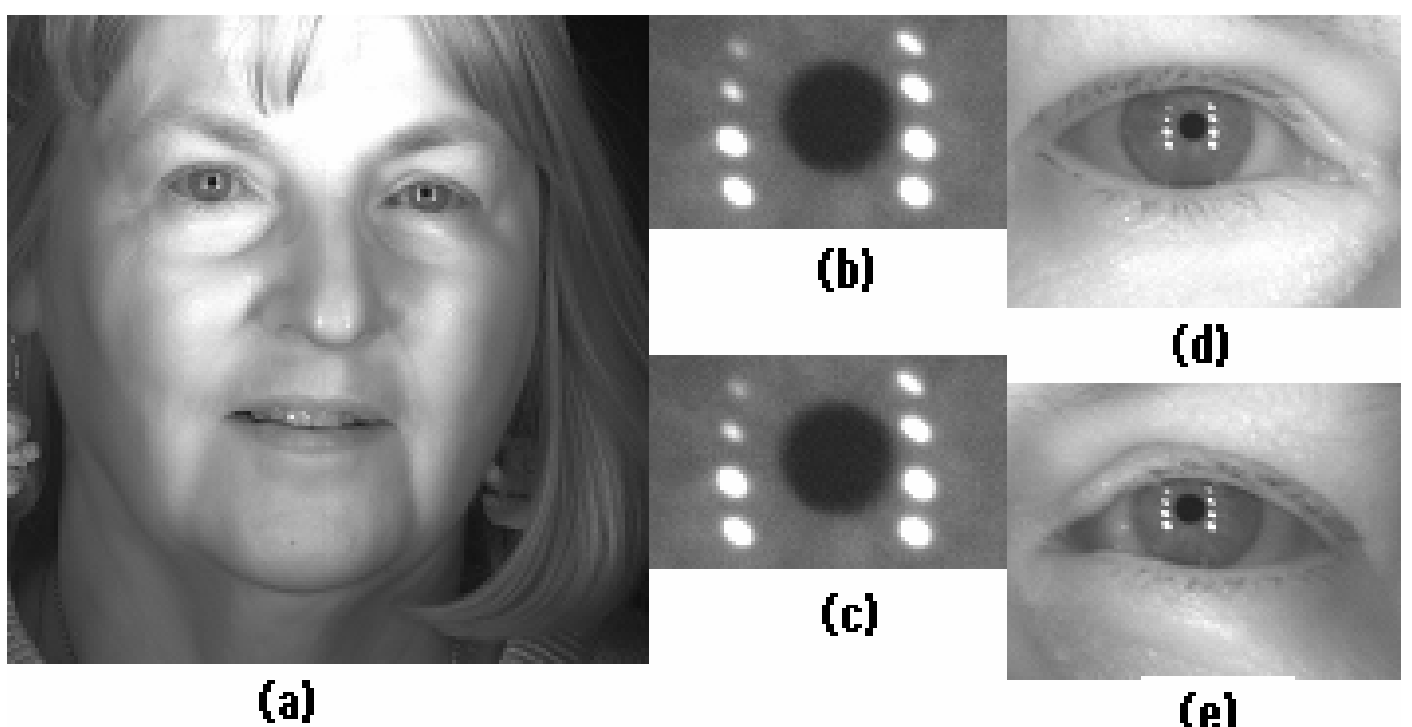

Figure 5.3: One frame of video and right and left eye images extracted from the frame. (a) frame of subject's NIR video with face appearing, (b),(c) right and left eye images extracted from the frame

A measure of caution is considered in view of the fact that parts and objects of the subject, such as skin, teeth, jewelry, and glasses among others may generate similar reflection spots that may confuse the recognition process. To reduce the computational burden and system errors, a verification step was taken after the reflection spots were found. For a pair of spots, the interval between them is either iris or pupil. In MBGC database, iris usually has gray level intensity between 80 and 120, and pupil has intensity between 20 and 45 . We used this specification to verify that the extracted image is indeed that of the eye. Although some "none-eye" images may still be extracted and verified, the pattern feature of these images would not affect the performance of the iris recognition system. 


\subsubsection{Pupil localization and center detection}

The iris part can be defined by two circular boundaries (Ma, Tan et al. 2004). They are the pupil boundary which is the inner boundary between the pupil and the iris, and the iris boundary which is the outer boundary between the iris and the sclera. These two circles are considered to be concentric for some traditional iris segmentation methods, although it has been generally accepted that those two circles usually do not share the same center. For conventional methods that rely on high image quality, the error generated by treating the two boundaries as concentric circles is relatively small. For the iris images obtained from NIR videos with lower image quality the two circular boundaries are assumed to be nonconcentric.

The step for defining the pupil boundary and center would follow after the eye images are extracted. We have found that, for a portion of the eye images, the shapes of the pupil boundaries were impacted by the reflection spots, such as in the example shown in Figure 5. 4. (a).

The pupil parts of the eye images extracted from the NIR videos are somewhat darker than other parts of the images. Furthermore, the pixels of the pupil part usually have similar gray level intensities. Thus, adaptive histogram method was used to find the

threshold for the pupil of each eye image. After studying the eye images, the average gray level intensity of pupil part for each eye image is found to be between 30 and 75 . 


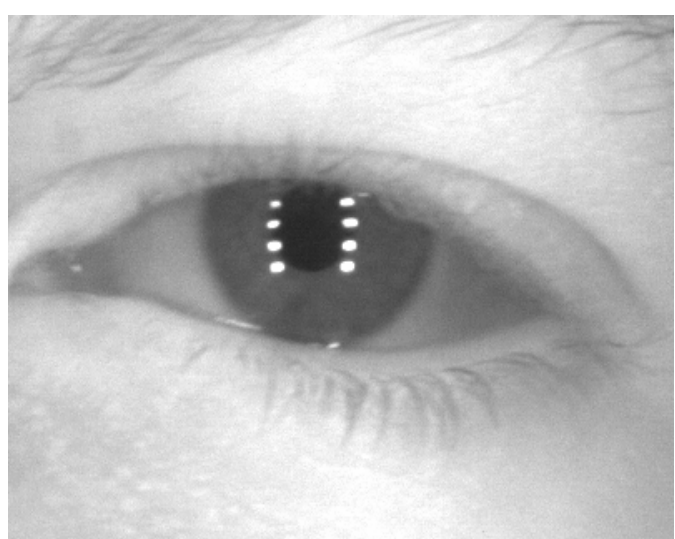

(a)

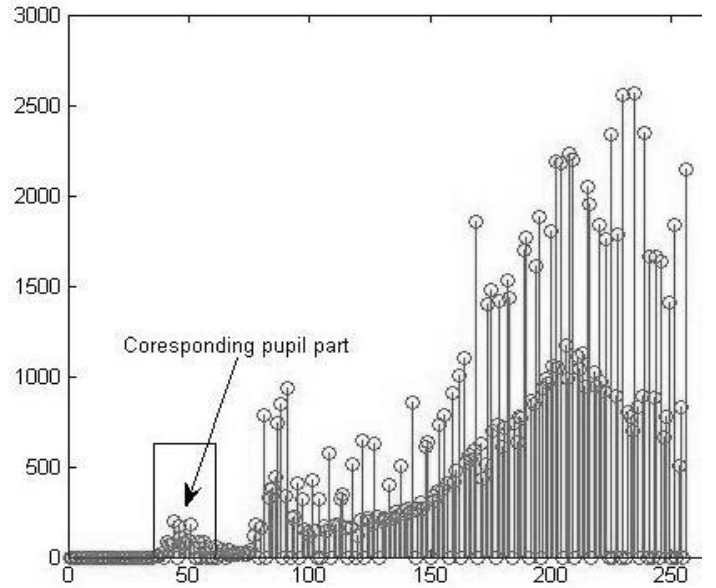

(b)

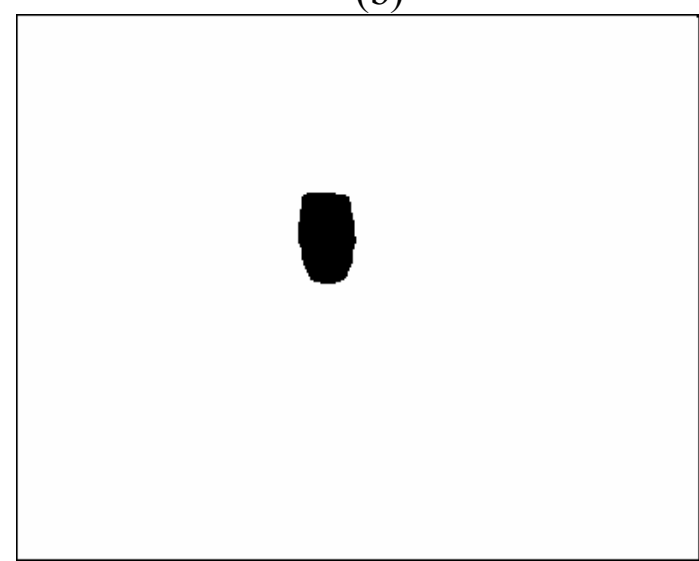

(c)

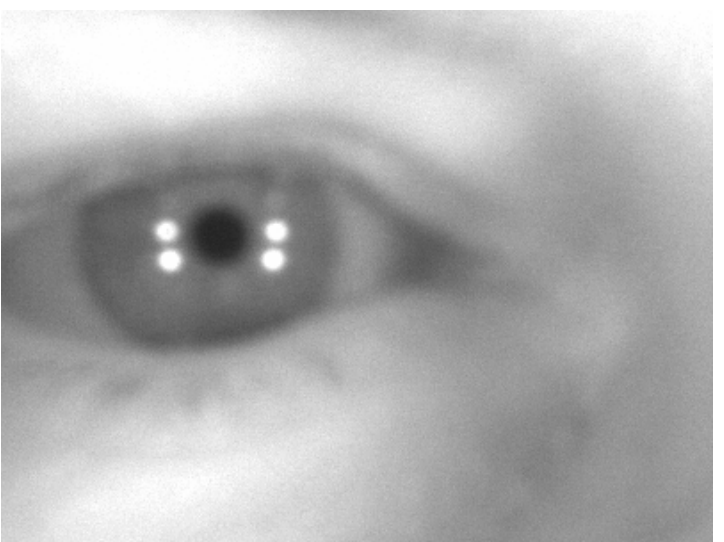

(d)

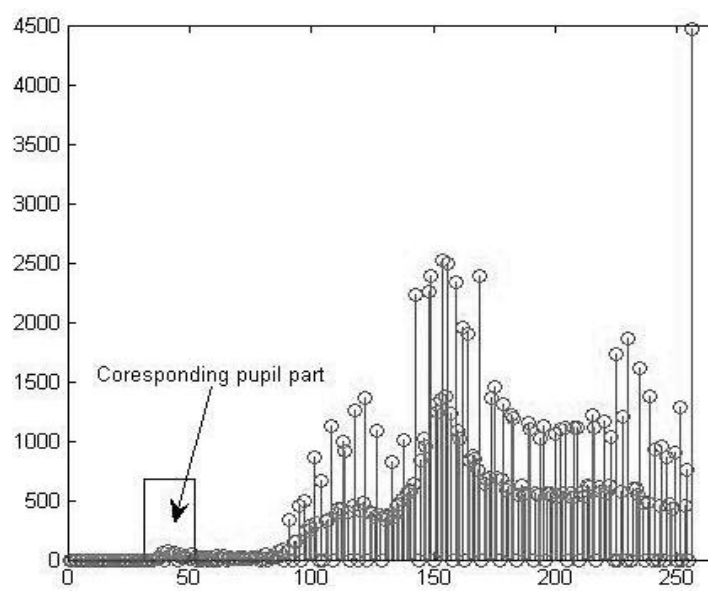

(e)

Figure 5.4: Adaptive histogram methods for defining the pupil. (a) eye image extracted from the video frame; (b) histogram analysis for image gray level intensities; (c) the extracted pupil part after transforming (a) to binary map; (d), (e), (f) demonstrate another case with the same organized order 
Thus, we have

$$
\begin{array}{ll}
D_{j}=G_{j}-G_{j-\frac{M}{2}} & \forall j \in(30,75) \\
G_{k}=\sum_{i=0}^{M-1}\left(I_{k-i}\right) & \forall k \in(25,75) \\
D_{T}=\operatorname{Max}\left(D_{j}\right) & \forall j \in(30,75)
\end{array}
$$

where $I_{K}$ is the gray level intensity, $G_{k}$ is the group intensity for every $M$ neighboring gray levels of the intensity histogram (here, $M=10$ ), $D_{j}$ is the derivative between two group intensities with an interval of $M$. From (3) we obtain $D_{T}$ which is the maximum value of group intensity derivative and with the same value of $\mathrm{T}$, the threshold can be obtained.

After transforming the eye image to a binary map, all the pixels that have gray level intensities greater than the set threshold $I_{T}$ are assigned a value of 255, and all the remaining pixels are set to 0 . Figure 5.4 (c), (f) show two examples of the obtained binary maps. The left limit (start) $X_{L}$ and right limit (end) $X_{r}$ of the pupil which refer to the black parts as in Figure 5.4 (d),(f) can be found in horizontal direction; and the top limit $Y_{T}$ and bottom limits $Y_{B}$ of the pupil can be found in the vertical direction. The center of the pupil can thus be defined as $\left(\left(X_{L}+X_{R}\right) / 2,\left(Y_{T}+Y_{B}\right) / 2\right)$, and the radius of the pupil can be formulated as $\operatorname{Max}\left(\left(X_{R}-X_{L}\right),\left(Y_{B}-Y_{T}\right)\right)$.

With this method, some errors may occur, which are caused generally by the heavy dark eyelashes, especially for those subjects who use cosmetic on their eyelashes. It is thus appropriate to investigate the area surrounded by the reflection spots. 


\subsection{Defining Outer Boundary and Eyelids}

\subsubsection{Defining outer boundary}

The outer boundary is defined as the boundary between iris and sclera. To delineate the outer boundary, the first step is to perform edge detection to get the edge map of the eye image. The second step is to apply the circular Hough transform.

The circular Hough transform is a voting based computational algorithm. For a 2D image, three parameters $(x, y, r)$ can define a circle, $(x, y)$ is the coordinates of the center of the circle, and $r$ is the radius of the circle. During the circular Hough transform, every point on the edge map would vote for a group of circles, the circle which gets the most votes would be the desired one. Although the circular Hough transform is a powerful algorithm, it also carries with it a heavy computational burden, and still the results may be impacted by the inherent noise effects. To improve the performances of the circular Hough transform, we have made some modifications towards both reducing the computational complexity as well as the noise effects.

With the generated edge map, instead of the traditional four directions Sobel edge detection, we modified the Sobel algorithm to only conduct the edge detection in the horizontal direction. Figure 5.5 illustrates the difference in the results of the two approaches. Figure 5.5.c shows the edge map generated by only performing the edge detection in the horizontal direction, in contrast to with Figure 5.5.b which is generated by edge detection in all 4 directions, the edge points have been significantly reduced, and the impacts from the upper and lower eyelid edges are likewise reduced. 


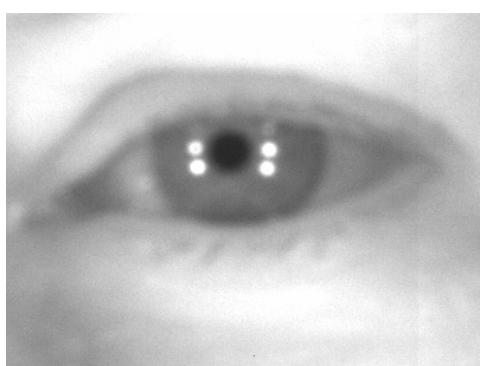

(a)

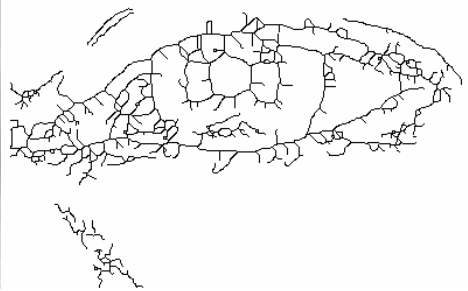

(b)

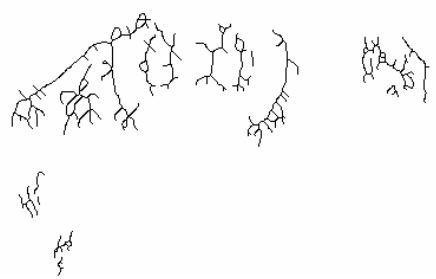

(c)

Figure 5.5: Sobel edge detection for four directions vs. the Sobel edge detection for horizontal direction: (a) original eye image; (b) resulting edge map using all four directions; (c) resulting edge map using only the horizontal direction.

After generating the edge map, based on the information we obtained from the previous steps, we also set some constraints for both the desired circle center and radius. First, the circle of pupil has been defined. Although the inner and outer boundaries are usually non-concentric, but it would be safe to assume that the outer center is located in the inner circle for most cases. And we also set lower limit of the radius of the outer boundary to be half of the interval distance between two lines of reflection spots, and the higher limit of the radius to be 5 times that of the radius for the pupil circle.

With these modifications, we have obtained the desired performance in search of circular shapes. Even in the presence of serious noise effects, the results obtained were still accurate. Figure 5.6 d. shows the results of the modified Hough transform applied to a blurred eye image, with constraints as indicated by Figure $5.6 \mathrm{~b}$ and Figure $5.6 \mathrm{c}$,

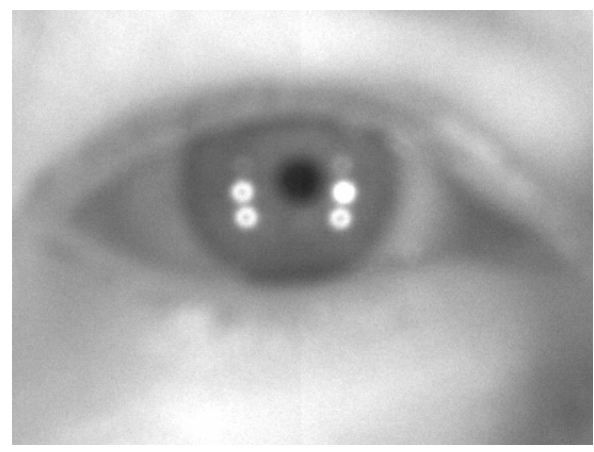

(a)

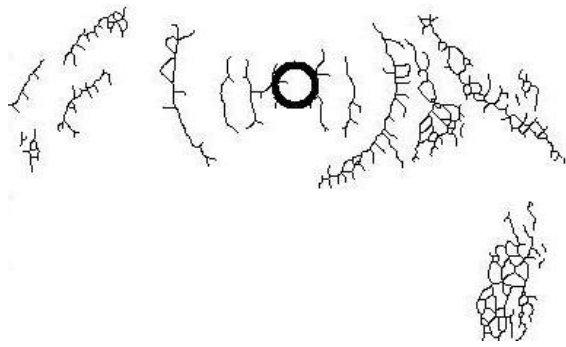

(b) 


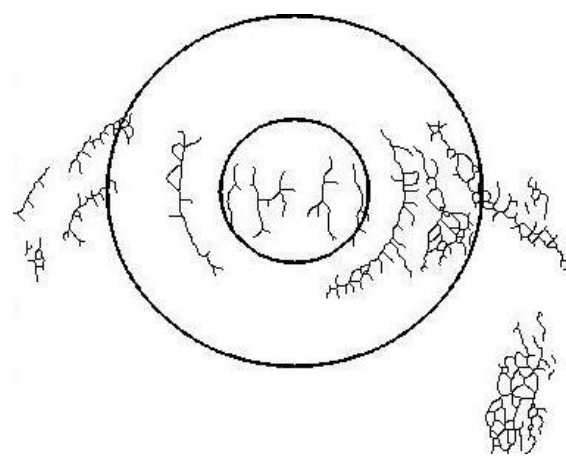

(c)

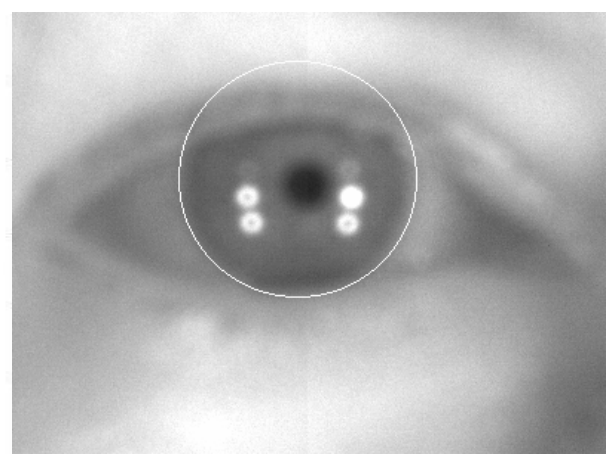

(d)

Figure 5.6: Example of modified Hough circular transform: (a) original eye image; (b) edge map, the center of outer boundary was constrained to be located in the inner circle; (c) edge map, where the edge points of the outer boundary were constrained to be located in the ring area between the two circles; (d) resulting outcome of the modified Hough circular transform.

\subsubsection{Detecting the Boundaries for the Upper and Lower Eyelids}

With the video-based iris recognition process, considerable eyelids occultation

often occurs. It is therefore important to detect such boundaries and to determine the extent of their occultation.

To detect the eyelids, the Hough line transform is used on the edge map of the eye image. However, with this type of algorithm, the key problem for dealing with noisy eye images is that the obtained edge maps are usually non-ideal. We have found that this problem was especially serious for the extracted eye images from videos. As shown in Figure 5.7. a, most edge points appearing on the map are undesired which are caused by the eyelids, reflection inside the iris and the texture of the iris patterns. To resolve this issue, an improved approach for detecting the eyelids is proposed in order to overcome the occultation of the eyelids.

Because the slops of the upper and lower eyelids are flat for most the length of the eyelid, our approach starts by conducting the edge detection only in the vertical direction. 
The generated edge map would thus highlight the desired edges of the eyelids. Figure 5.7.a is one such example. Figure 5.7.b shows the reflection area we obtained by expanding every found reflection point to a $5 \times 5$ mask. Thus, the detected reflection map can remove most of the edge points caused by reflections. Figure 5.7.c is the edge map after removal of the reflection.
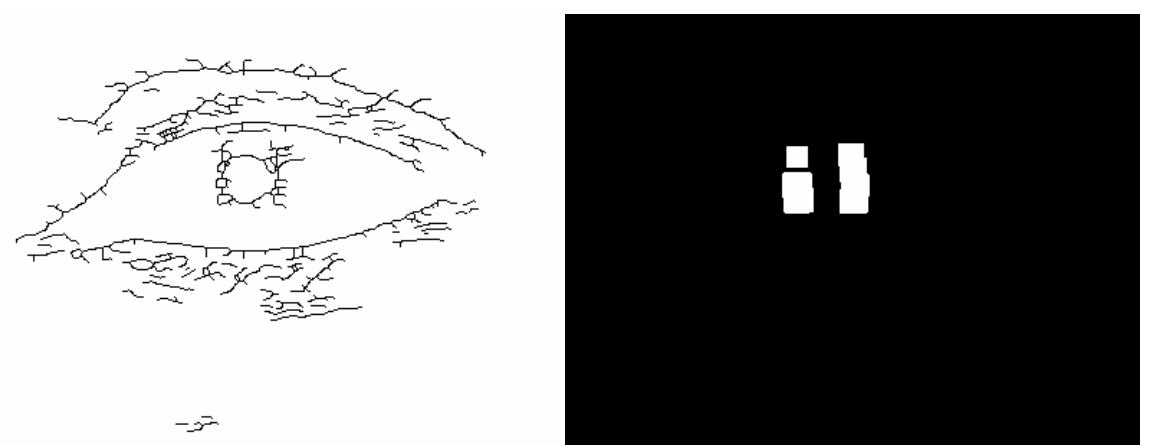

(a)

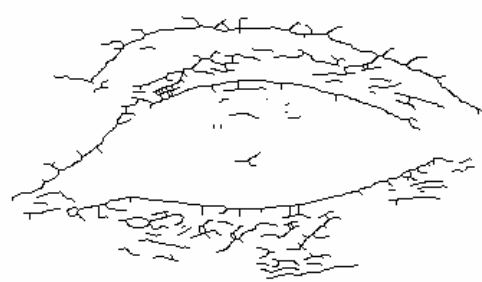

(c)

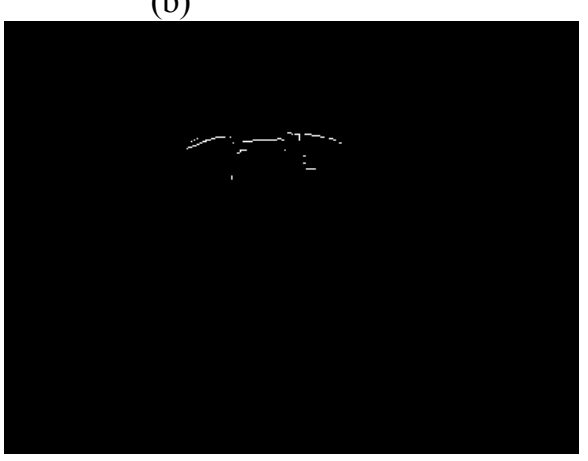

(d)
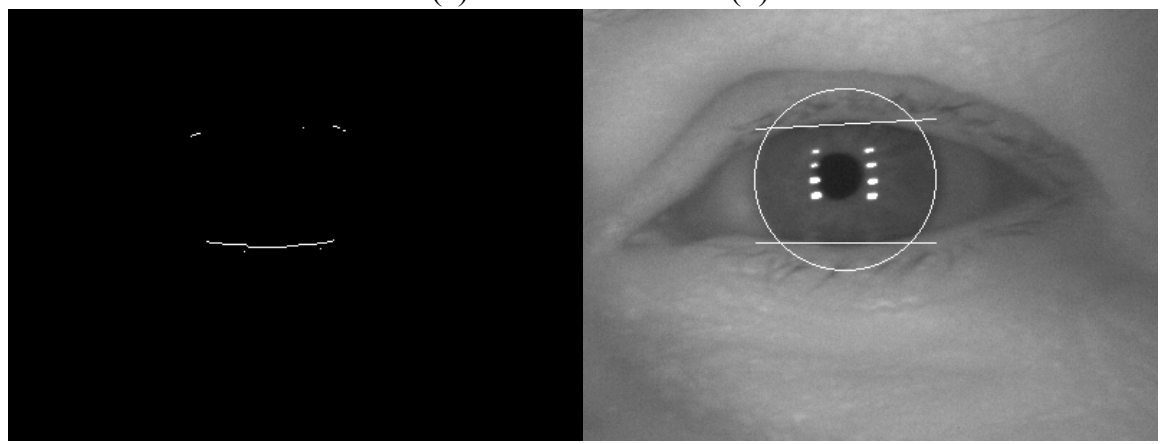

(e)

(f)

Figure 5.7: Example of the proposed method for detecting upper and lower eyelids: (a) edge map found with only the vertical direction operator; (b) detected reflection area; (c) edge map after removing undesired edge points; (d) detected points which are on the edge of iris and upper eyelid; (e) detected points which are on the edge of iris and lower eyelid; (f) resulting outcome of the iris detection through eyelids detection. 
To distinguish the points which were only on the edges between iris and eyelids, we first pick a patch of area to calculate the average gray intensity of the iris. As can be seen from Figure 5.8, we choose the area just below the pupil, since this area is most unlikely to be affected by the reflections or overlap with the eyelids. As such, the size of the patch is chosen to be $15 \times 15$ in size, and the upper boundary of the patch is $R / 10$ below the pupil's lower boundary, where $\mathrm{R}$ is the radius of outer boundary.

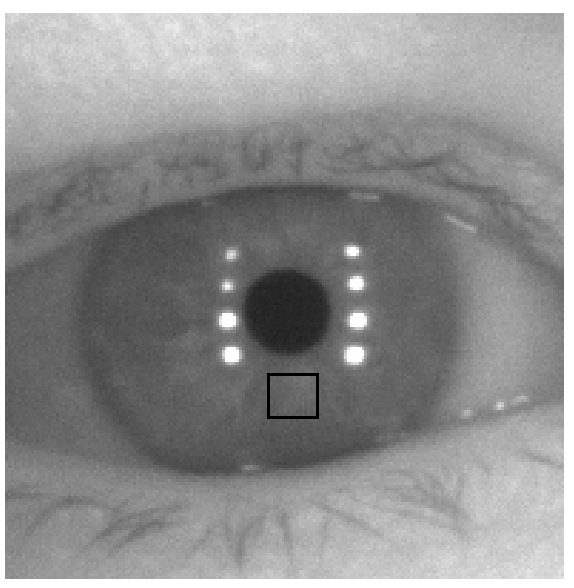

Figure 5.8: Example of obtaining average gray intensity

With the information of outer boundary circle, we made rays which start from the center of the circle and toward every point on the upper or lower arc of the circle (central angle $\theta=120$ ). In Figure 5.9.c, for each ray, if a edge point was found, $M$ points beyond this point in the ray's direction would be verified by $I_{A}$, if $N$ of points can be considered as points on iris, the found edge point would be ignored, and searching would go on until reach the circle boundary. In this work, we set $M=8$ and $N=5$. For the example shown earlier in Figure 5.7, part (d) shows the verified edge points for the upper eyelid, and (e) the edge points for the lower eyelid. 
After the verified edge map was generated, the Hough line transform is then used.

Since most of the undesired edge points would be removed before this step, the results of the Hough line transform appears to be more accurate and more tolerant of noise.

For some cases, it would be more accurate to describe the eyelid with two lines. In our approach, after the first line was detected, we remove the edge points on that line, then, conduct Hough transform again, if there are enough votes, another line would be determined. An example of these cases was shown in Figure 5.9.

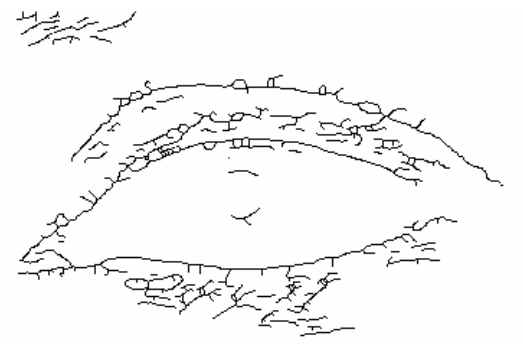

(a)

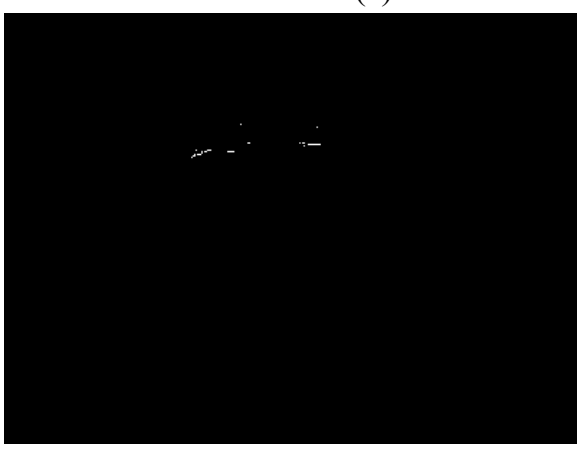

(c)

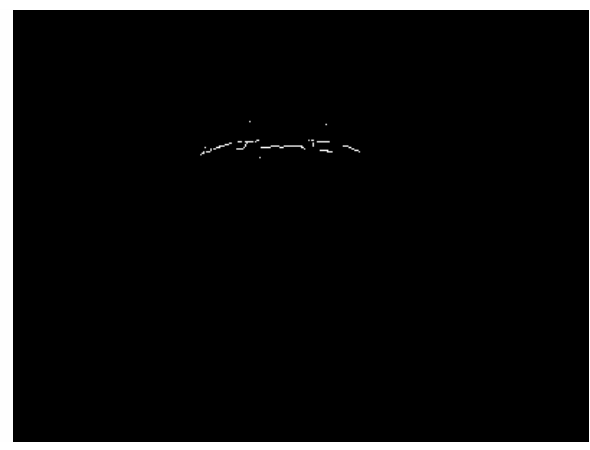

(b)

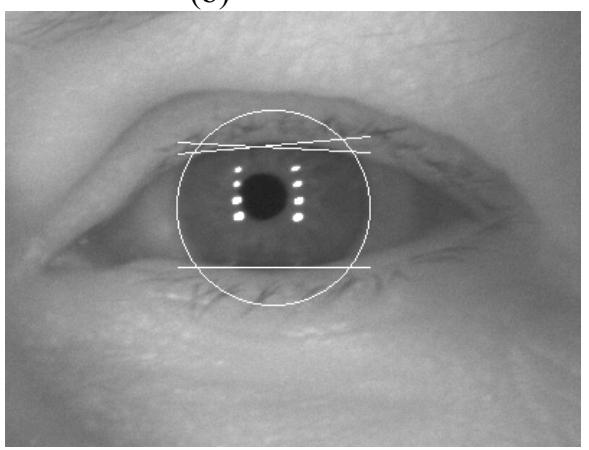

(d)

Figure 5.9: Example for detecting eyelid with more than one line: (a) original edge map; (b) verified edge map before first Hough line transform; (c) edge map before the second Hough line transform towards upper eyelid; (d) final results of the iris extraction process 


\subsection{Verification and Correction of Outer Iris Boundary}

Although outer iris boundary was assumed to be circular for most of the previous research studies on iris segmentation, it has been pointed out that human iris boundaries are usually noncircular. For same cases, describing the iris boundaries as circles could yield inaccurate segmentation results and impact the verification performance eventually. This problem is especially crucial for video based iris recognition such as with the IOM system, because of the recurring off angle effects during video capturing and also because of the relatively small size of the iris image obtained from the frames. To overcome this problem, a new method is proposed.

This new method is based on three steps: (1) circle center correction, (2) multiple circle (or Arc) searching, (3) multiple arcs and lines connection.

Although Hough circular transform is very powerful among circle searching algorithms, some noise effects such as off angle, too many edge points outside of the iris caused by the eyelashes or skin, and the noncircular shape of outer iris boundary, all can lead to inaccurate results of the transform. Figure 5.10.a is one such example, where the black circle is the result of the traditional first Hough transform. The outer boundary can not be correctly described.

The first step of our proposed method follows after conducting Hough Circular Transform for detecting the outer boundary. In this step, we need to find the correct center of iris to verify the original circle center found from the first Hough circle transform. If the difference is greater than an adaptive threshold, we consider that the outer iris circle needs to be corrected. For this study, we set the adaptive threshold to be $R / 6$, where $R$ is the radius for outer iris circle. We adopted the pupil centers which 
were found before (this step described in section 5.3.2) as the correct iris center. Figure 5.10.b indicates those two centers.

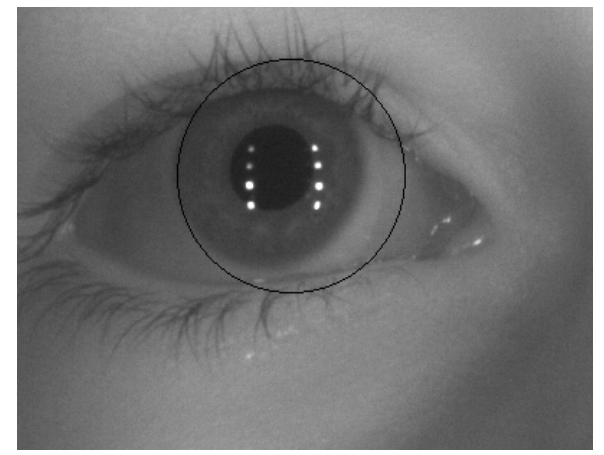

(a)

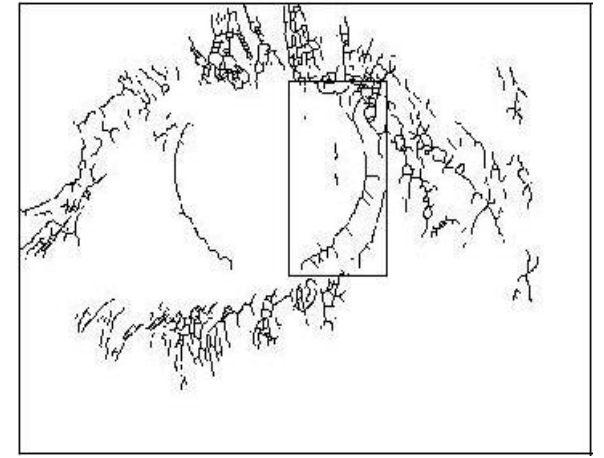

(c)

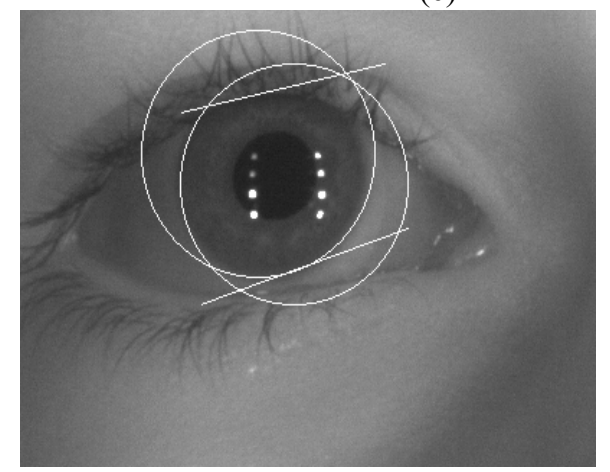

(e)

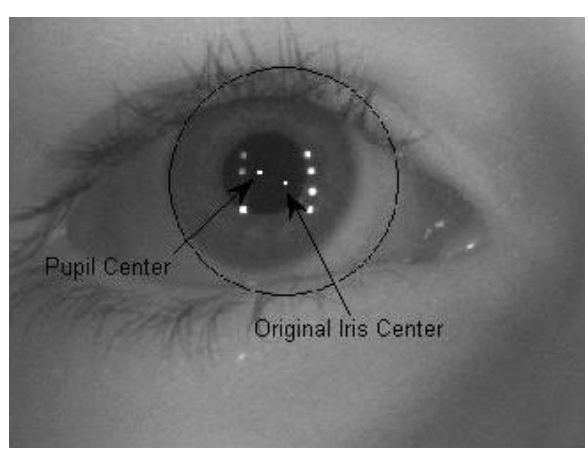

(b)

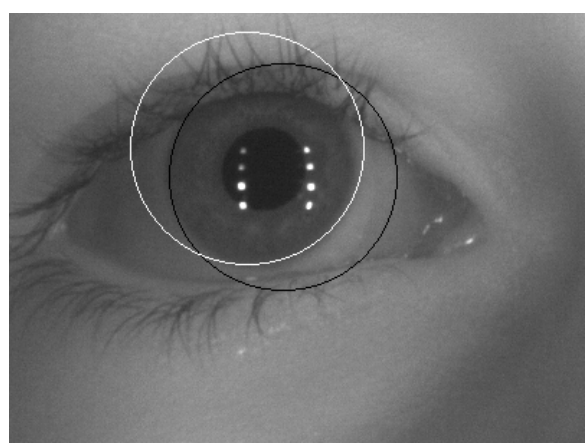

(d)

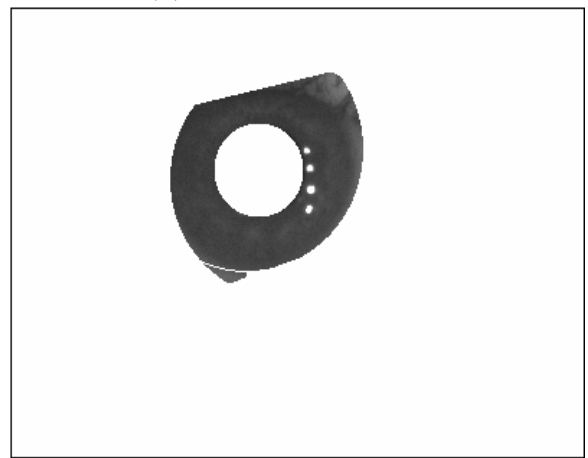

(f)

Figure 5.10: Example for defining the noncircular iris boundary. (a) The black circle is the result of the 1st Hough transform; (b) shows the center of pupil and center of original iris center; (c) the square shows the target region which was defined by two centers (d) the white circle is the result Hough circle of target area;(e) shows the intersection of two circles with the detected lines for upper and lower eyelids; (f) is the result after the noncircular correction

Then a target area would be selected for conducting Hough circular transform again. Based on the study of inaccurate results of Hough transform, the desired iris 
boundary is usually inside the Hough circle. The reason of that is the edge points outside the iris usually vote for a larger circle and off angle iris usually smaller than its actual size. Thus, the target area was expected to be the region between the real iris center and the arc on the opposite side of original circle. The method introduced in section 4.6 is adopted to search for the targeted area (rectangle) for conducting the Hough transform.

In this study, we set the width of the rectangle to be $4 R / 5$, and the height to be $2 R$. Another Hough transform will be performed on the edge points inside the target rectangle. Since the Hough transform is based on voting, to avoid uncertain outcomes, we set an adaptive voting count threshold such that if the highest voting count is below this threshold, the circle will not be generated. The white circle shown in Figure 5.10.d is an example of the results obtained because of this step.

The intersection of two circles could be used to describe the noncircular iris. The upper and lower eyelids lines would be used here to remove unexpected eyelids and eyelashes. Thus, as Figure 5.10 (e) and (f) shown, the final result of the detected boundary consists of multiple arcs and lines, and would be more accurate for describing noncircular iris boundaries. Three other examples that have been generated using this method are as shown in Figure 5.12.

Comparing with other iris segmentation methods such as active contour, with respect to describing noncircular iris boundaries, our method is more tolerant to noise effects. Because we still use Hough circular transform to detect circles (arcs), even when the edge points were partially missing or incorrect, the Hough transform results remained the same. 


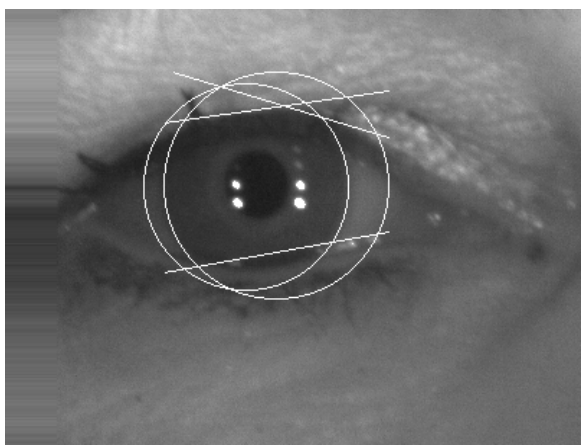

(a)

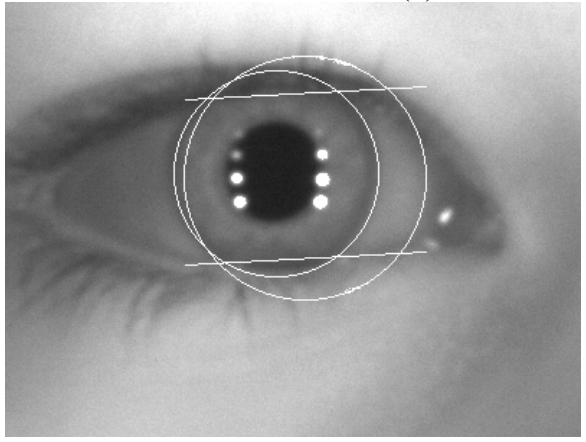

(c)

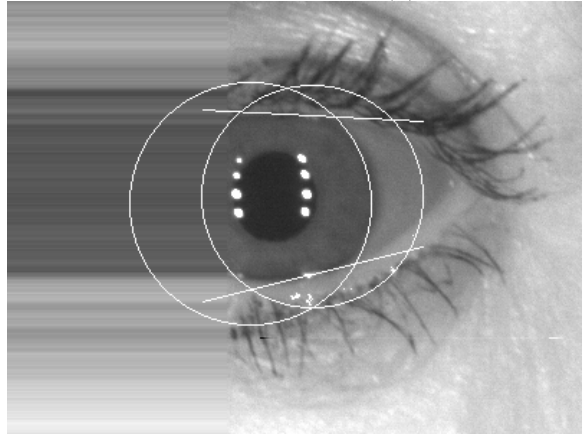

(e)

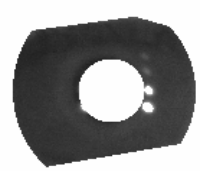

(b)

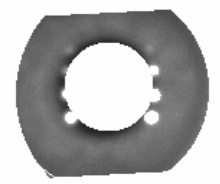

(d)

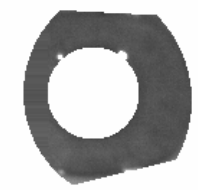

(f)

Figure 5.11 : Three other examples, (a), (b) show one example which 2 arcs and 3 lines were used together to describe the boundary; (c), (d) show Hough transform can be corrected through this process, the larger circle was the original one obtained from the first Hough transform; (e),(f) show how this method deal with the eye image which is out of video frame, for adjusting the eye image to our segmentation system, we fill the out range part of image with the same pixels of last line or column.

\subsection{Experimental Results}

Experimental evaluation of this method involved the use of NIR-face-video data from Multiple Biometric Grand Challenge (MBGC) database- version 1. All the NIR videos were captured by acquisition equipments provided by Sarnoff with the IOM system which is similar to the one introduced in . There are 149 videos in the NIR-face- 
video portion of MBGC-version 1. Each video captured one person's face video when the person walked through the portal. The resolution of the video is $2048 \times 2048$, and the frame rate is $15 \mathrm{frames} / \mathrm{s}$. There is no specific location for the positioning of the face during video capturing, thus the eyes may appear in any part of the video frames. Each video consisted of about 20 frames, among these frames, as indicated before, there are about 1 or 2 frames that could have been captured within the desired capture volume. For the rest of the frames, images of the eye tend to suffer from the effects of defocus and blurring with different degrees, and there are several other frames that do not contain the eye portion. With the eye image extraction used in our approach, the image size is $400 * 300$ for each eye. The pixels across the iris are about 120 for average, and for the pupil, the pixels range from 12 to 80 , which is still considered significant.

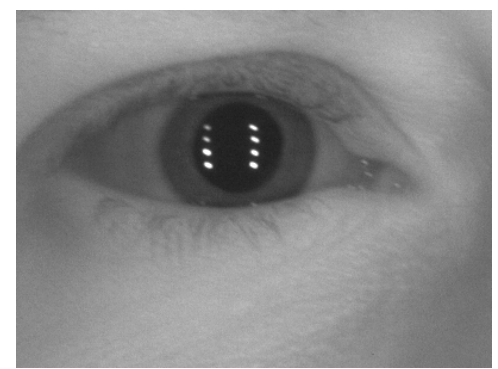

(a)

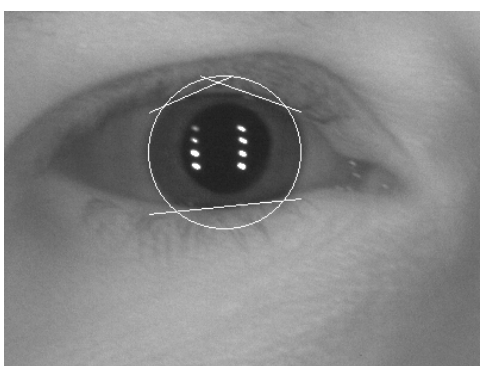

(b)

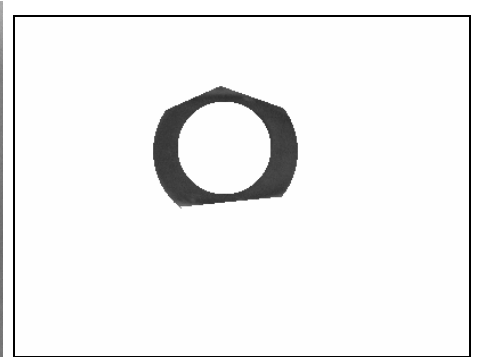

(c)

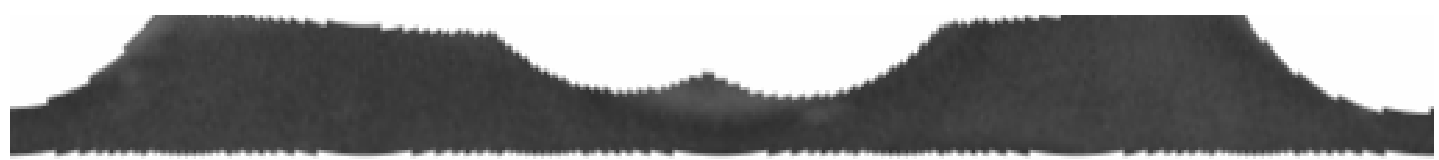

(d)

Figure 5.12: An example of successful segmentation: (a) eye image extracted from the video; (b) detected iris boundary; (c) result of the segmented iris; (d) polar transform. 


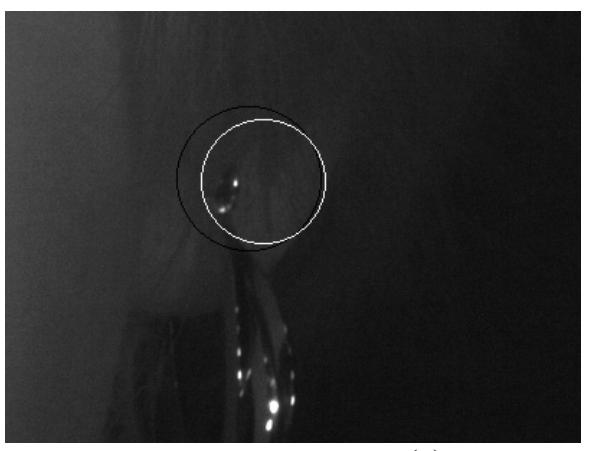

(a)

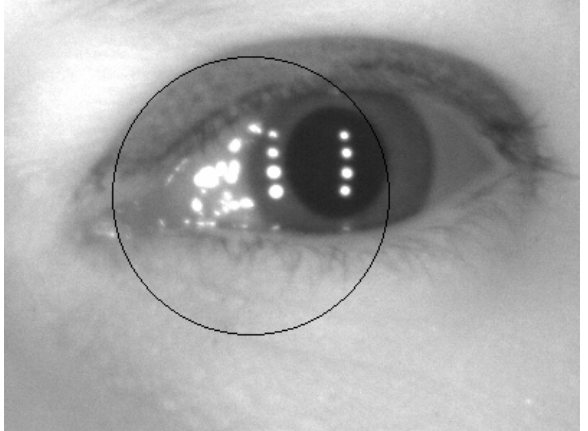

(c)

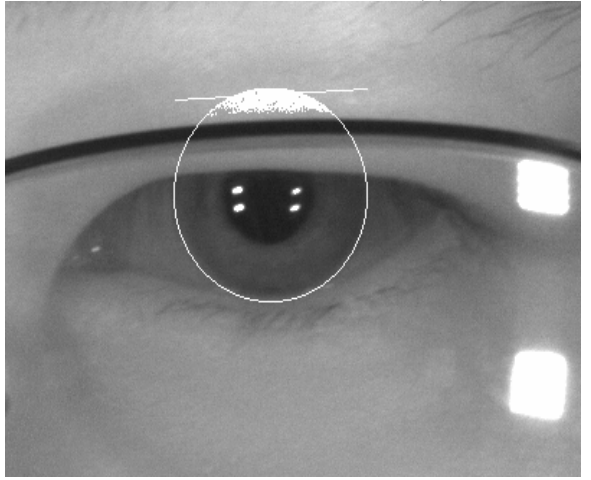

(e)

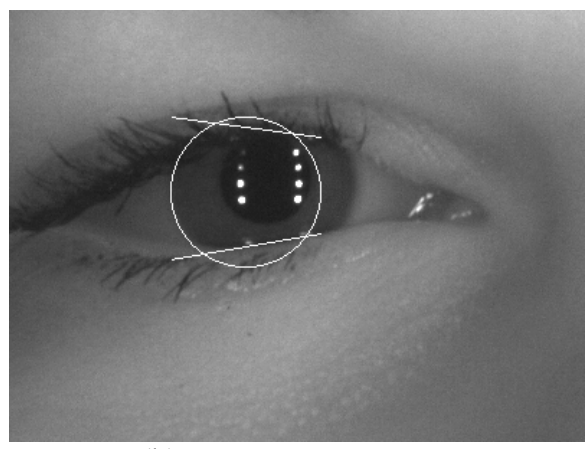

(b)

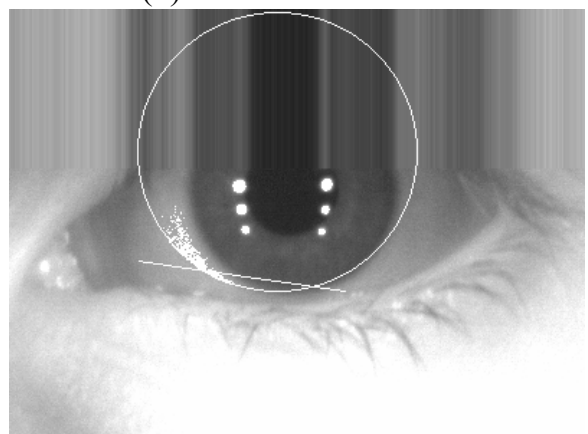

(d)

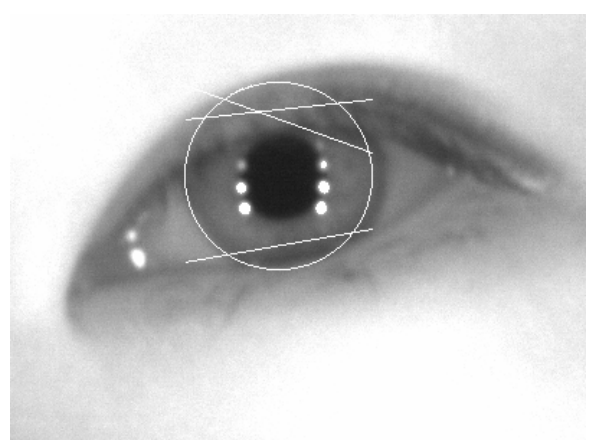

(f)

Figure 5.13: Error examples of segmentation results due to other effects: (a) caused by jewelry; (b) caused by heavy eyelids occultation; (c) caused by unexpected reflection; (d) caused by the eye is out of the video frame range;(e) caused by eyeglasses;(f) caused by unexpected reflection; (d) caused by the eye is out of the video frame range;(e) caused by eyeglasses; (f) caused by blurred edge between eyelid and iris

Also, we have obtained some inaccurate results, as shown in Figure 5.14. Because of taking iris video in the less constrained environment, some unexpected situations seldom happen, but most of those cases can be avoided if some mild adjustments were applied to subjects during the verification process. 
Since this study is focused on segmentation, and because the size of NIR-facevideo data is relatively small, we conducted the experiment all the eye images extracted from 149 video, and assessed the segmentation results in terms of accuracy as shown in Table.1.

Table 5-1: Experiment results based on different levels of eye image extraction constrains.

\begin{tabular}{|c|c|c|c|c|}
\hline $\begin{array}{c}\text { Test } \\
\text { result }\end{array}$ & $\begin{array}{c}\text { Total } \\
\text { extracted } \\
\text { eye images }\end{array}$ & $\begin{array}{c}\text { Average eye } \\
\text { images for } \\
\text { each subject }\end{array}$ & $\begin{array}{c}\text { Total } \\
\text { errors }\end{array}$ & Accuracy \\
\hline Test 1 & 657 & 4.4 & 38 & $94.216 \%$ \\
\hline Test 2 & 891 & 5.9 & 57 & $93.603 \%$ \\
\hline
\end{tabular}

With the system designed using $\mathrm{C}++$, the average eye image extraction time for one frame of video is about $0.02 \mathrm{~s}$, and the average processing time for one iris image is about $0.71 \mathrm{~s}$. The total extracted eye images vary the different extraction conditions initially imposed. With less strict conditions we obtained more eye images from the videos. As more eye images are extracted, the more defocus or blurred images would be enrolled to the verification system, which would hinder the iris verification process. As Table.1 revealed, the accuracy just slightly drops as more images were obtained, which indicate that our approach remains nonetheless tolerant to noise. 


\section{Iris Recognition Experiments on Video Images}

\subsection{Introduction}

This chapter reports on the results obtained through experiments that have been conducted on the less-constrained video based iris recognition process. Some key modifications have been made to improve the recognition accuracy rate in terms of both the false acceptance rate (FAR) and the false rejection rate (FRR).

In the less-constrained video-based iris recognition process, the test or subject iris videos/images and the enrolled iris images are acquired with different acquisition systems. To accelerate the verification or identification process and hence improve the convenience of the users, the test iris images are taken with NIR video while the subjects are on the move (Phillips 2009; Zhou, Du et al. 2009). The enrolled iris images are taken with the conventional acquisition system to ensure the quality of the iris images. In the matching step, the verification/identification result is given by comparing the similarity distance of encoded signature from test images with each of the signature dataset from the enrolled iris images.

The process of the less-constrained iris recognition is demonstrated in Figure 6.1. In these experiments, the subject videos are from the NIR-face-video dataset of Multiple Biometric Grand Challenge (MBGC) database (version 1) provided by the National Institute of Standards and Technology (NIST), the enrolled iris images are from the NIRiris-still dataset of the MBGC database (version 1). The subject videos are taken with a prototype of the Sarnoff Iris on-the-Move (IOM) system which is introduced in Chapter 
III. The enrolled iris images are taken with the LG IrisAccess 2200 which is one of the commercial iris recognition systems based on the conventional acquisition system.

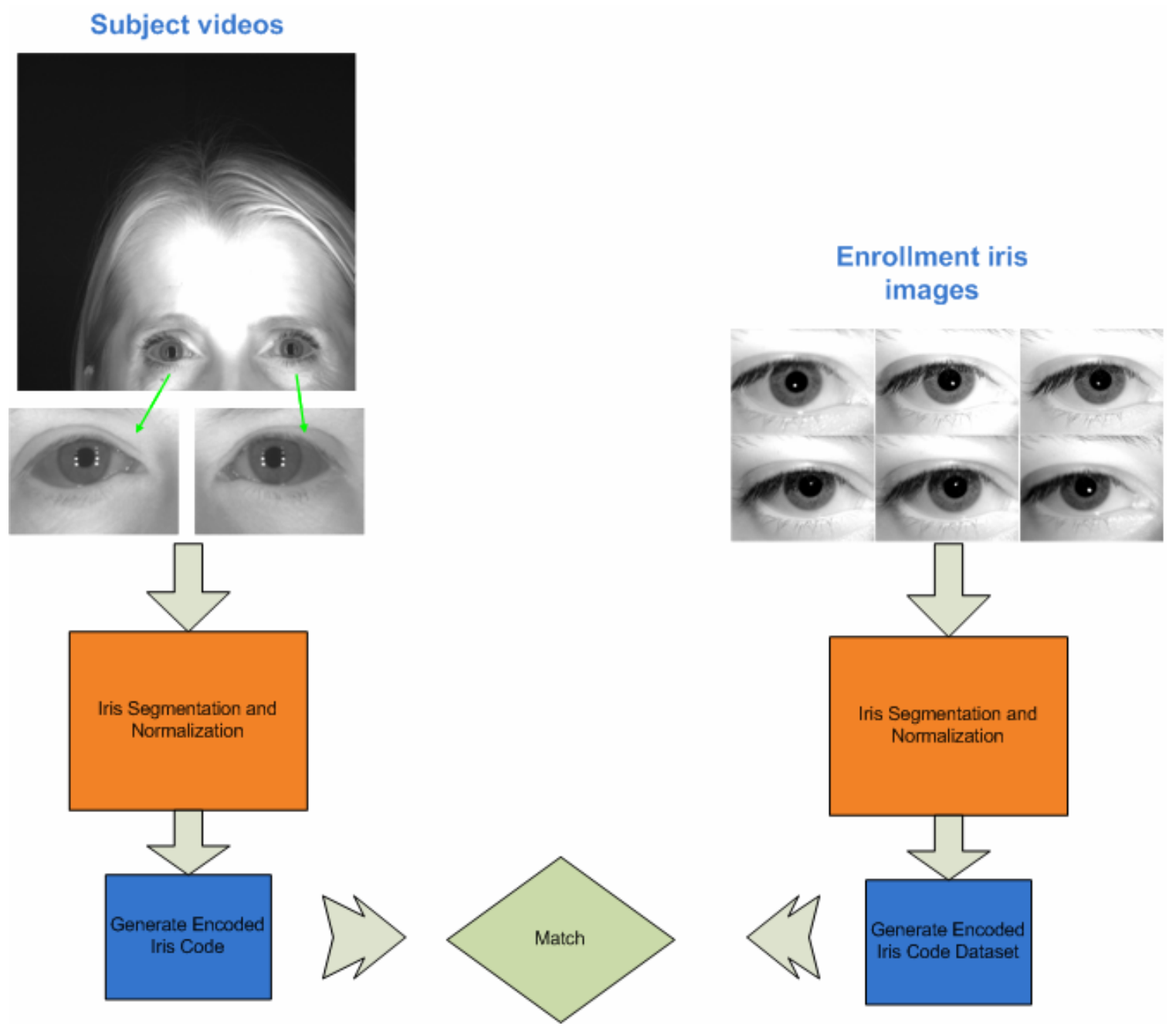

Figure 6.1: Process of less-constrained iris recognition based on video

The test iris images are extracted and segmented with the segmentation approach that was used on the less-constrained video-based iris recognition process as detailed in chapter III. Then the enrolled iris images are segmented with an implementation that was based on the ICE baseline of Biometric Experimentation Environment (BEE)(X. Liu 2005). Some modifications have been made to improve the segmentation accuracy. The 
ICE baseline of BEE is a conventional Daugman-like iris recognition approach implemented by the University of Notre Dame similar to the original approach implemented by Libor Masek (Masek, 2003).

The amount of test iris images and enrolled iris images are listed in Table 6.1. The test iris images are acquired from 149 NIR videos with the less-constrained segmentation approach. The enrolled iris images are a sub-set of the NIR-iris-still dataset.

Table 6-1: Amount of the test iris images and enrolled iris images

\begin{tabular}{|c|c|c|c|}
\hline $\begin{array}{c}\text { Image } \\
\text { group }\end{array}$ & Left & Right & Total \\
\hline $\begin{array}{c}\text { Test Iris } \\
\text { Images }\end{array}$ & 139 & 172 & 311 \\
\hline $\begin{array}{c}\text { Enrolled } \\
\text { Iris Images }\end{array}$ & 109 & 109 & 218 \\
\hline
\end{tabular}

To ensure the compatibility in matching the two sets of iris images, each iris image needs be transferred from a circular ring in Cartesian coordinates to a rectangle in polar coordinates for normalization (Daugman 2001). The images segmented with noncircular correction method are excluded as those segmentation results can not be normalized as a circular ring. For the test image collection, multiple iris images can be extracted and segmented from each subject eye, and for the enrolled iris image collection, the NIR-iris-still dataset also provides multiple still images with different optical focus for each subject eye. Because this study focuses on the recognition process, some of the extracted iris images yielding degraded image qualities are excluded, maintaining only those images with better quality extracted from the same subject/eye. A single NIR still 
image with good quality for each subject eye is enough to perform this recognition process.

\subsection{Optimization in Normalization and Encoding}

The resolutions of the enrolled iris images and test iris images can be different due to the different acquisition methods, optical focus, and distances between subjects and camera. The dilation of the pupil can also change the iris region significantly. Each segmented iris image needs to be normalized to the same dimension before encoding it to a signature code. In this study, the segmented iris image with a shape of circular ring in Cartesian coordinates is transferred to the shape of a rectangle in the polar coordinates with the rubber-sheet algorithm proposed by Daugman (Daugman 2004).

The polar plot which yields a signature of the object is as illustrated in Figure 6.2, where Equations 6.1, through 6.3 apply. Point $\mathrm{O}$ in Figure 6.2 defines the pupil center, $r$ is a value on the unit interval, and $\theta$ is an angle in the interval $[0,2 \pi]$. Thus, each point on the iris $I(x(r, \theta), y(r, \theta))$ in the Cartesian coordinates can be defined as $I(r, \theta)$ in the polar coordinates, regardless of the difference in the image resolution and pupil dilation. In Equations 6.2 and 6.3, $\left(x_{p}(\theta), y_{p}(\theta)\right)$ and $\left(x_{i}(\theta), y_{i}(\theta)\right)$ are the two points along $\theta$ located on the inner iris boundary and the outer iris boundary, respectively. Both concentric and non-concentric iris shapes can be converted using polar plots.

$$
\begin{gathered}
I(x(r, \theta), y(r, \theta)) \longrightarrow I(r, \theta) \\
x(r, \theta)=(1-r) x_{p}(\theta)+r x_{i}(\theta) \\
y(r, \theta)=(1-r) y_{p}(\theta)+r y_{i}(\theta)
\end{gathered}
$$




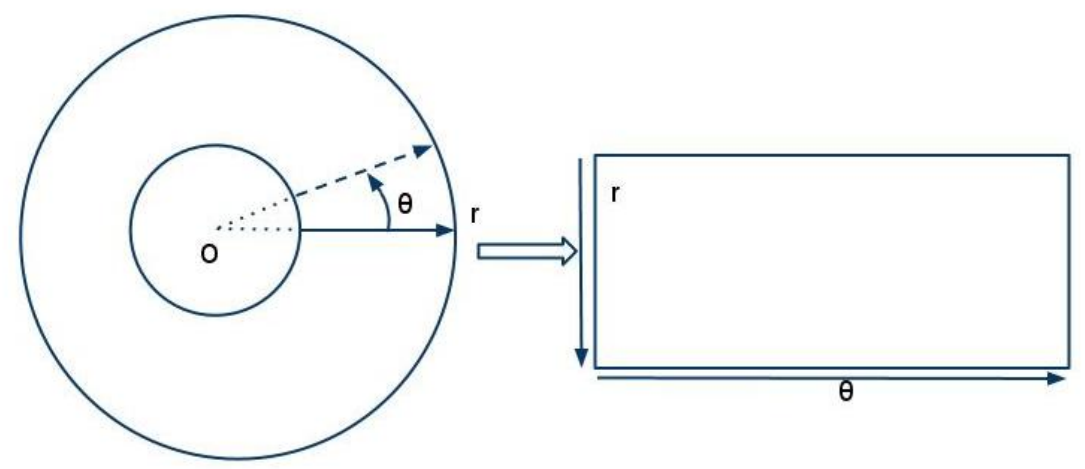

Figure 6.2: Illustration of normalization step with the rubber-sheet algorithm

Two examples of polar plots are shown in Figure 6.3. Figure 6.3.(a) is a test iris image extracted from the NIR-face-video; (c) is the result image of (a) after normalization, where the white part (set $=1$, in the image) is the detected noise region for eyelid, eyelash, specular reflection; (e) serves as a mask template of (c) to indicate the location of the noise, where regions with noise are set to be 1, and the noise free regions are set to be 0 . The Figure 6.3 (b) is an enrolled image; (d) is the result image of (b) after normalization; (f) serves as a mask template of (d).

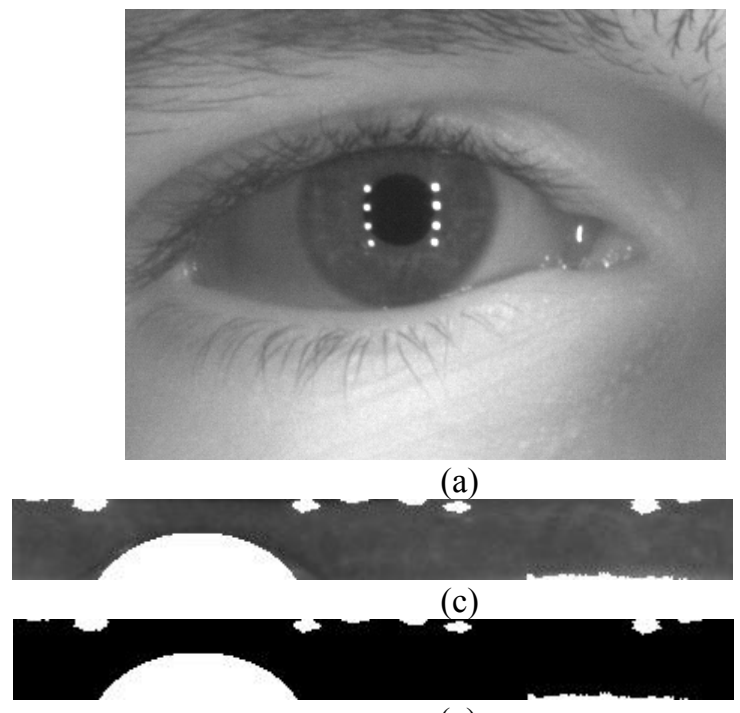

(e)

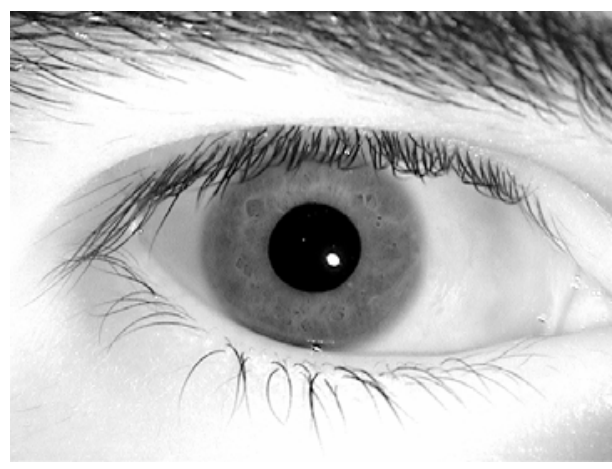

(b)

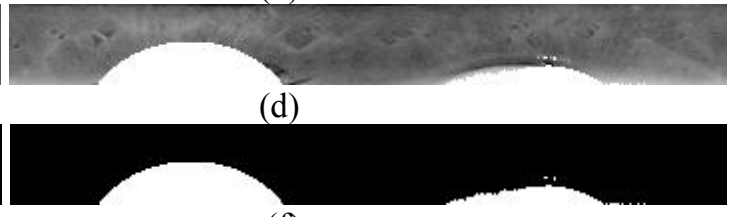

(f)

Figure 6.3: Normalization examples for test iris image and enrolled iris image 
In the conventional iris recognition, image enhancement is rarely performed after the normalization process. As the image quality is good enough to contain enough feature details of the iris, and image enhancement may in fact negatively impact the recognition results. However, for the conventional iris recognition, the image qualities of the test image are usually low due to lighting and off-focus effects. We have made some modification to the traditional recognition steps by performing histogram equalization on the result image of the normalization step, as given by equation 6.4 .

$$
I^{\prime}(i, j)=\frac{t_{h}-t_{l}}{T_{h}-T_{l}} \times I(i, j)
$$

Where $I(i, j)$ is the gray intensity of the pixel $(\mathrm{i}, \mathrm{j}), t_{h}$ and $t_{l}$ are the highest and lowest intensity value of the input image. $T_{h}$ is the highest intensity value in the result image and $T_{l}$ is the lowest intensity, in this study we set $T_{h}=1$ and $T_{l}=0$. To eliminate the noise effects, we use the average values of $1 \%$ of pixels with the highest intensity value for $t_{h}$, and $t_{l}$ is the average value of $1 \%$ of pixels with the lowest intensity value.

Figure 6.4 illustrates two cases of the histogram equalization process. Figure 6.4 (a) is a normalization result test image without performing the histogram equalization; (b) is the corresponding result after the enhancement obtained through the histogram equalization process. Figure 6.4 (c), (d) are normalization result of an enrolled image before and after performing histogram equalization, respectively. For both of these cases, the images after the transformation show clearer certain feature details not visible in the original images, especially for the case of the test image. 
(a)

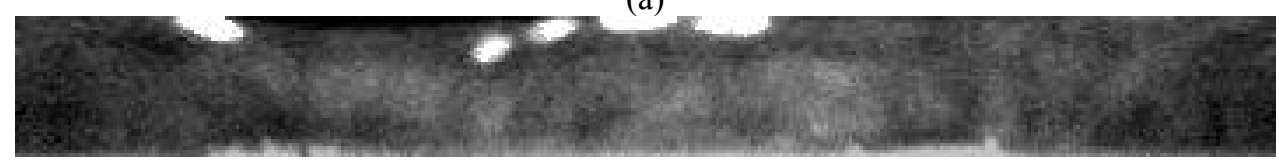

(b)

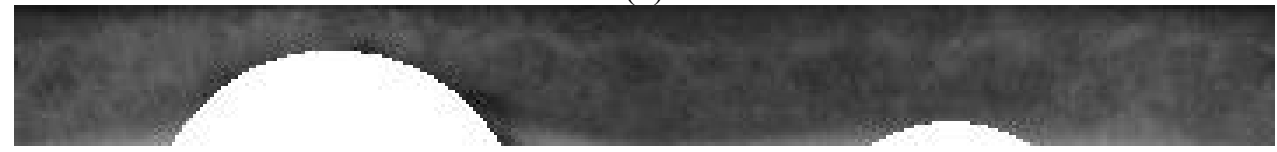

(c)

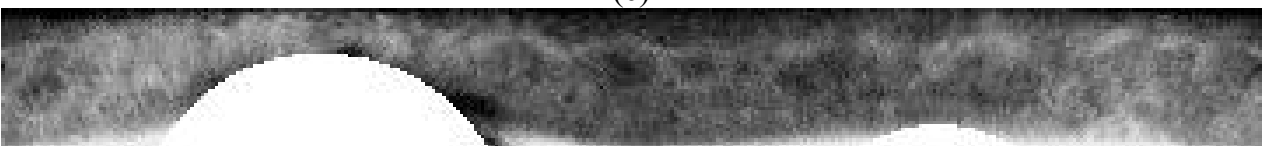

(d)

Figure 6.4: Iris images before and after contrast enhancement

In the encoding part, a 1-D log-Gabor wavelet filter is used to encode the normalized iris images. As shown in Figure 6.5, the process starts by performing a 1D FFT on each row of the normalized iris image. The result of FFT transform is then multiplied with a 1D Log-Gabor filter as given by Equation 6.5.

$$
G(f)=e^{\left(\frac{-\left(\log \left(f / f_{0}\right)\right)^{2}}{2\left(\log \left(\sigma / f_{0}\right)\right)^{2}}\right)}
$$

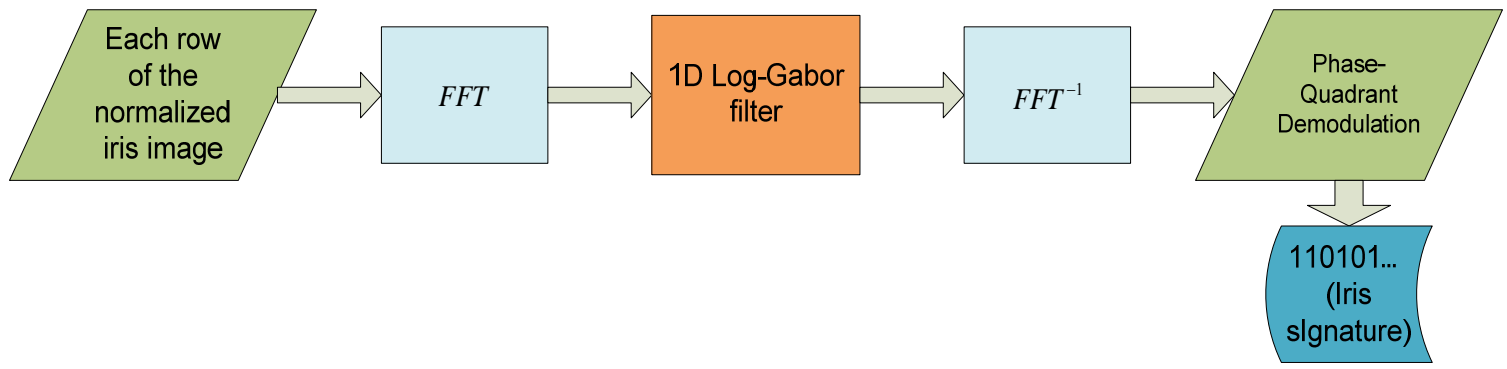

Figure 6.5: Encode process 
In equation 6.5 the $\sigma$ value defines the bandwidth of the filter, and $f_{0}$ refers to the center frequency of the filter. An inverse FFT transform would then be performed on the result of Log-Gabor filter. As afinal step, a phase-quadrant demodulation method (Daugman 2004), as illustrated in Figure 6.6, is used to encode the iris to a set of binary codes. Each input to the demodulation method $H_{\{\text {real, image }\}}$ contains the real part and the imaginary part. The phase of the $H_{\{\text {real, image }\}}$ is thus quantized to a pair of codes.

$$
S 1=\left\{\begin{array}{l}
1 \Leftarrow \operatorname{sign}(\text { real })>0 \\
0 \Leftarrow \text { else }
\end{array} \text { and } S 2=\left\{\begin{array}{l}
1 \Leftarrow \operatorname{sign}(\text { image })>0 \\
0 \Leftarrow \text { else }
\end{array}\right.\right.
$$

Each of the input complex number can be encoded to a pair of code (S1, S2), with the four sets of the values as $(1,1),(1,0),(0,0),(0,1)$. Thus the final result of the encode process would be a binary code with a double width of the normalized iris image. In this study, we set the dimension of the normalized iris image as $40 \times 360$, and the final encoded signature would be a binary signature with a size $40 \times 720$.

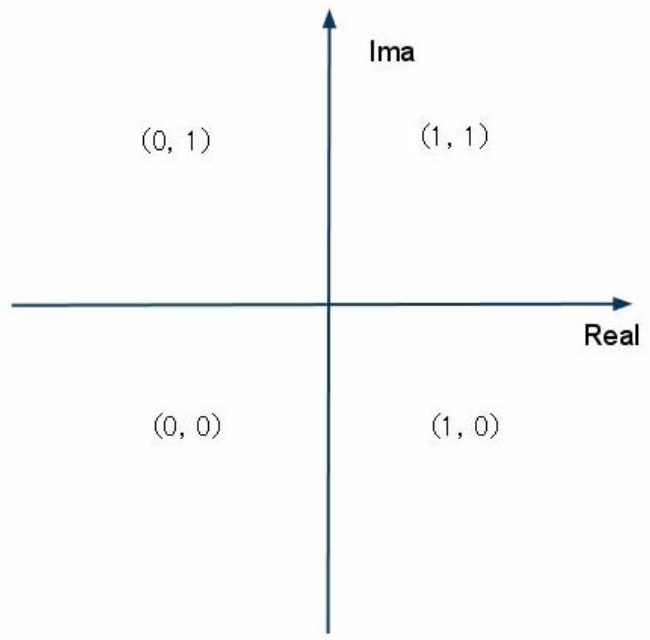

Figure 6.6: Phase-Quadrant Demodulation method 


\subsection{Experiments and Analysis}

The Hamming Distance is used in this case to match the similarity distance between two encoded signatures. The Hamming Distance can be obtained using Equations 6.7, and 6.8.

$$
\begin{gathered}
H D=\frac{1}{N-\sum_{i=1}^{N}\left(\operatorname{Tn}_{i} \text { OR En } n_{i}\right)} \sum_{j=1}^{N}\left(\left(T_{j} \otimes E_{j}\right) * V_{j}\right) \\
V_{j}=\left\{\begin{array}{l}
1 \Leftarrow T n_{j}=0 \text { And } E n_{j}=0 \\
0 \Leftarrow \text { else }
\end{array}\right.
\end{gathered}
$$

where $T_{j}$ denotes the $j^{\text {th }}$ bit of the encoded binary iris signature for the test image, $E_{j}$ denotes the number $j^{\text {th }}$ bit of the encoded binary iris signature for the enrolled image. $N$ is the total number of bits on the iris signature, $T n_{j}$ refers to the $j^{\text {th }}$ bit of the mask template, which indicates the locations of all the noise regions. We have $T n_{j}=0$ when the $j^{\text {th }}$ bit of the signature is not on the noise region, otherwise, $T n_{j}=1$.

The lower the Hamming distance is, the more similar the two iris images are. Although two images taken with an identical eye may not exactly be the same, they usually yield the lowest Hamming distance. A threshold can be used to make the decision if two irises are identical. In our study, the test iris images are extracted from the NIRface videos. After the video extraction, segmentation and quality evaluation, 305 images are used as the test dataset. Those test images belong to 146 individual eyes, and 85 persons. There are 218 iris images which were collected with high image qualities which are used as the enrolled images. Those 218 iris images belong to 211 individual eyes and 107 persons. 
Table 6-2: Statistics of the test iris images and enrolled iris images. Note that for some individuals only one eye image is provided

\begin{tabular}{|c|c|c|c|}
\hline $\begin{array}{c}\text { Image } \\
\text { group }\end{array}$ & Total & $\begin{array}{c}\text { Individual } \\
\text { eyes } \\
\text { detected }\end{array}$ & $\begin{array}{c}\text { Individual } \\
\text { persons }\end{array}$ \\
\hline $\begin{array}{c}\text { Test Iris } \\
\text { Images }\end{array}$ & 305 & 146 & 85 \\
\hline $\begin{array}{c}\text { Enrolled } \\
\text { Iris Images }\end{array}$ & 218 & 211 & 107 \\
\hline
\end{tabular}

The comparison is between each of the test iris image with each of the enrolled iris images. The total amount of comparison trials is 66,490 , which is including 66,192 inter-class comparisons and 298 intra-class comparisons. Intra-class is defined as two iris images whith one being the test image from the NIR video, and the other the high quality still image belonging the identical eye, and the intra-class refers to those pairs of images belonging to different eyes. Figure 6.7 shows the distributions of the Hamming distances generated by the inter-class pairs and the intra-class pairs in amounts and percentages.

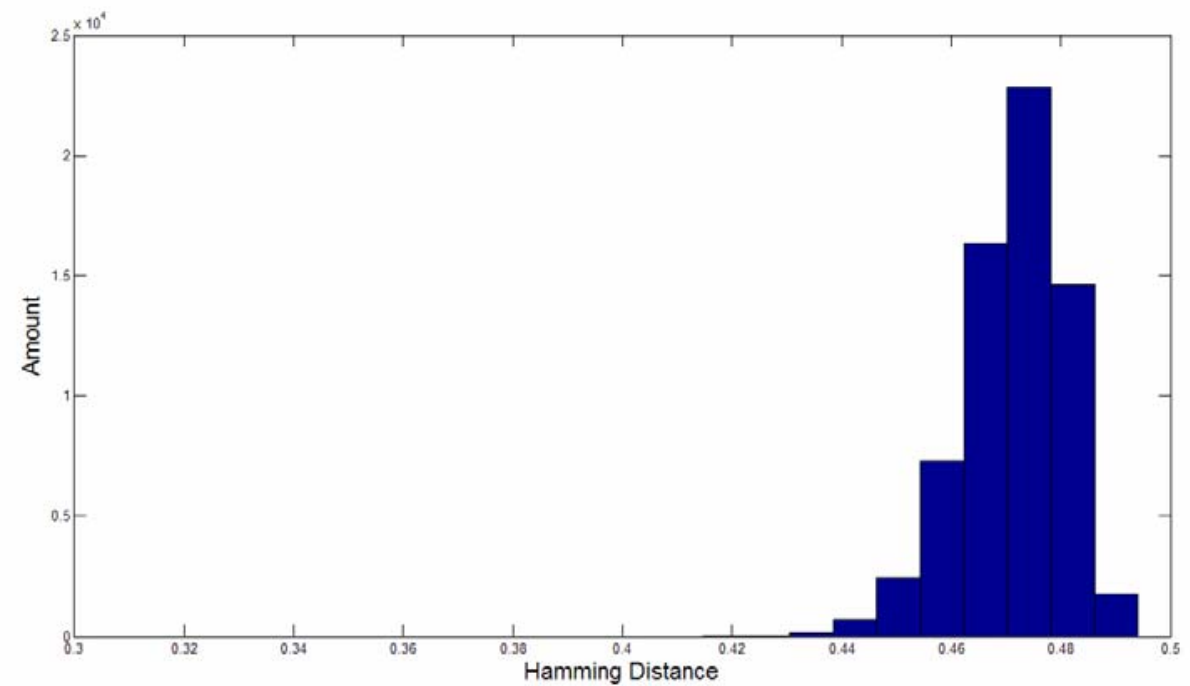

(a) 


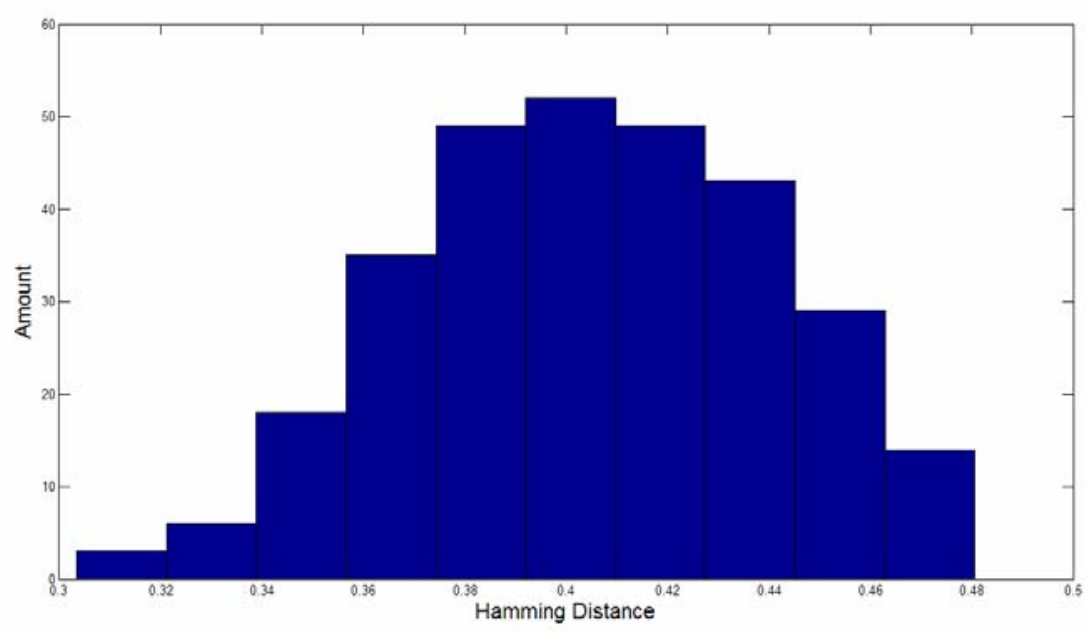

(b)

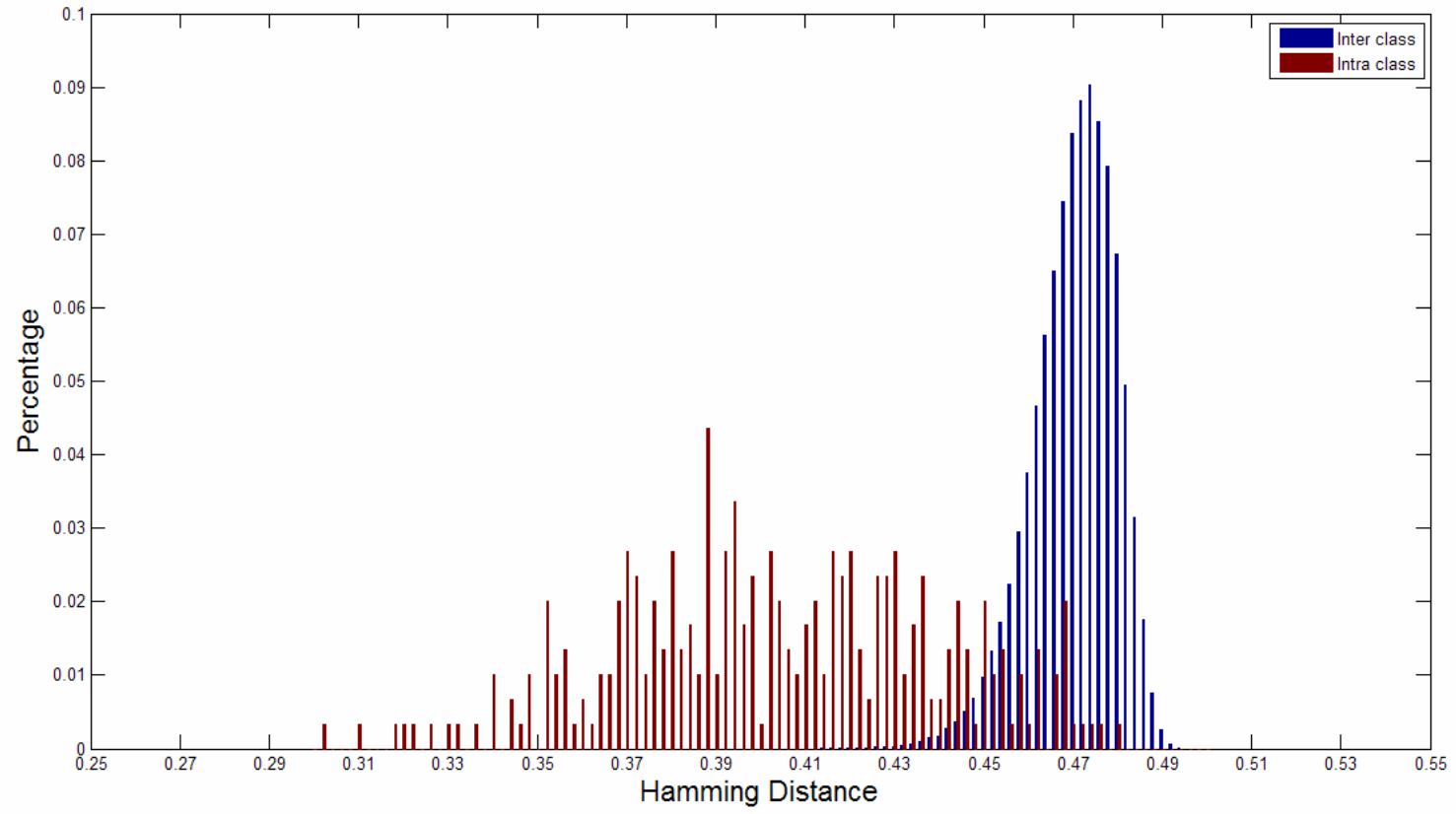

(c)

Figure 6.7: Distributions of Hamming Distance for inter- and intra-class comparisons

As can be seen from Figure 6.7, the range of the Hamming distance in the intra class, which is from 0.3 to 0.48 , is larger than the distance range for inter class, which is from 0.42 to 0.49 . The reason is that the image quality for the test images vary, if the quality is low enough, even when comparing with the enrolled image acquired from the same eye, the similarity between them would be just like comparing a pair of images 
from the inter class process. Figure 6.7 (c) illustrates the Hamming distance distribution in percentage for both of the groups. It does also show as would have been expected an overlap between the two distributions, but it does clearly portray a bimodal distribution.

The recognition rate on the basis of FRR and FAR with respect to the corresponding Hamming distance is shown in Figure 6.8. The FRR and FAR are as defined by Equations 6.9 and 6.10, respectively.

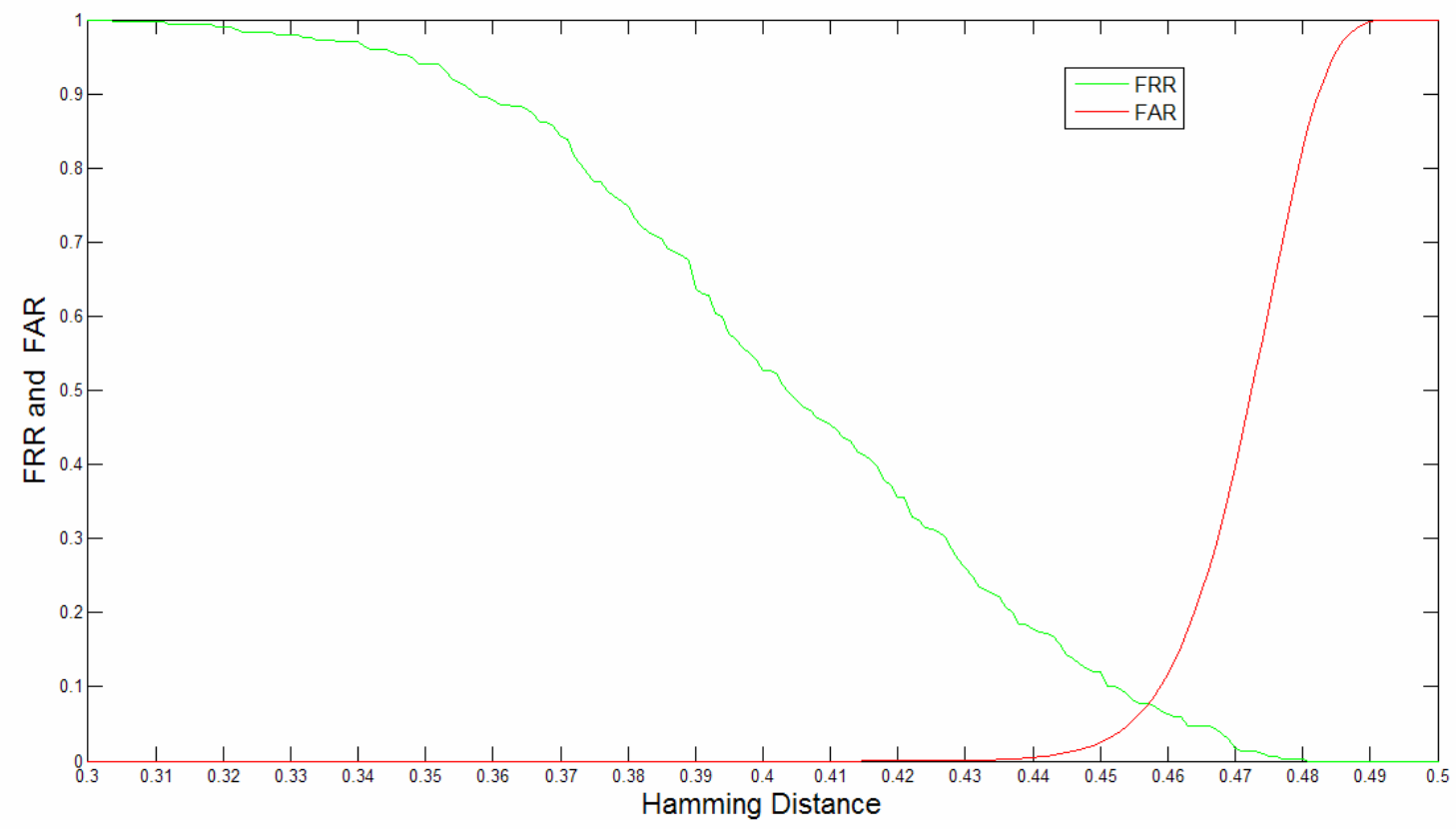

Figure 6.8: False Rejected Rate (FRR) and False Accepted Rate (FAR) vs. Hamming Distance

$$
\begin{gathered}
F R R=\frac{\text { The amount of falsely rejected qualified trials }}{\text { Total trials should be granted }} \\
F A R=\frac{\text { The amount of falsely accepted imposter tries }}{\text { Total trials should be rejected }}
\end{gathered}
$$

The improvement in recognition rate of the optimization step can be verified by the relative operating characteristic (ROC) curves shown in Figure 6.9. The best performance is given by the process which relied on histogram equalization on both test 
and enrolled classes, which refers to the red curve. It yields lower FAR with a certain FRR rate than other two curves.

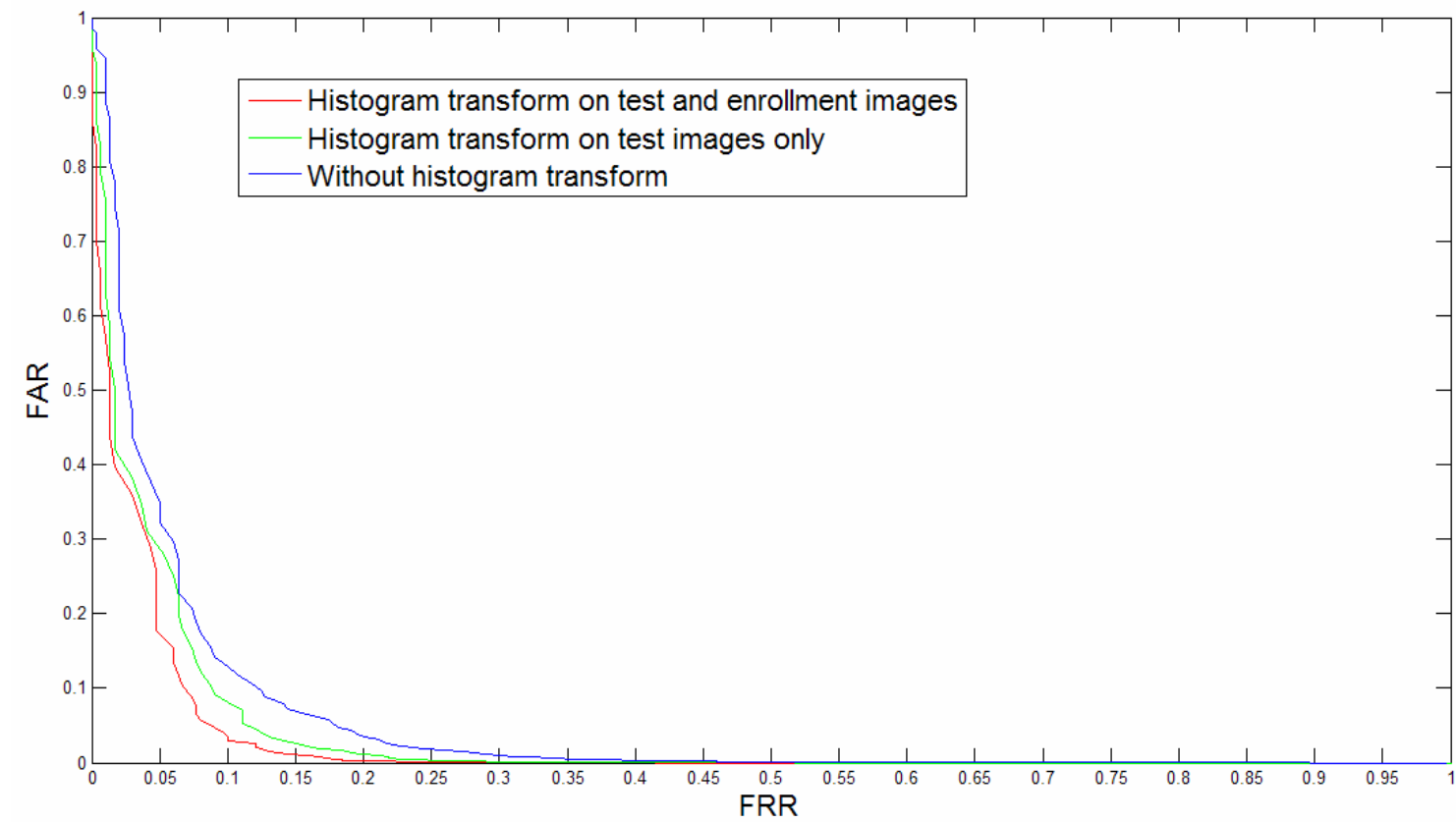

Figure 6.9: ROC curves for three cases as the one with histogram transform on the test and enrolled images, the one with the histogram transform on the test images only and the one without this histogram enhancement

As can be seen in Table 6.3, when the threshold of for the Hamming distance is 0.414, the FAR is 0, which means none of the 66,192 trials from inter class is accepted, with a tradeoff of an FRR rate at $41.61 \%$. When the threshold is set to be 0.418 , the FAR is $3.0215 \mathrm{e}-005$, with a better FRR at $37.92 \%$.

Because multiple iris images for the same eye can be extracted from different video frames for each person. If we treat a group of iris images as one subject, which means the lowest Hamming distance of such a group is registered to that eye, at the Hamming distance threshold of 0.418 , the FRR for the eye would be $18.85 \%$, and the FAR is 0 . 
Table 6-3: FRR and FAR with corresponding Hamming distance

\begin{tabular}{|c|c|c|}
\hline $\begin{array}{c}\text { Hamming } \\
\text { Distance }\end{array}$ & FRR & FAR \\
\hline 0.414 & 0.4161 & 0 \\
\hline 0.415 & 0.4128 & $1.5108 \mathrm{e}-005$ \\
\hline 0.416 & 0.4060 & $1.5108 \mathrm{e}-005$ \\
\hline 0.417 & 0.3960 & $3.0215 \mathrm{e}-005$ \\
\hline 0.418 & 0.3792 & $3.0215 \mathrm{e}-005$ \\
\hline
\end{tabular}

In the identification process, all the test images extracted from left and right eyes of one person can be seen as one group of images, and the lowest Hamming distance of that group would be registered to that person. Thus, at the Hamming distance threshold of 0.418 , the FRR for the person would be $17.64 \%$, and the FAR is 0 . These are viewed as quite accurate given the most important measure is that of the FAR, and that such results are based on the less constraining environment where subjects are on the move. 


\section{Conclusion and Discussion}

This dissertation introduced an innovative approach towards less-constrained iris biometrics. Two major contributions are covered, one is an award-winning segmentation approach towards the less-constrained iris biometrics taken under visible lighting conditions, and the other is a pioneering research towards segmentation and recognition approaches of less-constrained iris biometrics based on the video of moving persons under different acquisitions scenarios.

This research endeavor achieved high performance on eye images from the UBIRIS.v2 database, which contained very realistic noise effects. The accuracy of the proposed approach was evaluated as part of the NICE.I contest, ranking the method with the sixth lowest error rate among 97 participants worldwide. This accuracy is augmented in merit by the fact that the processing speed of the proposed approach is near real-time, requiring only $0.83 \mathrm{~s}$ to perform all the required steps for a final iris segmentation.

Furthermore, the proposed approach relied on an effective search for the sclera area of the image. A threshold of saturation value (the HSI model) was obtained by calculating the biggest group derivative of the histogram of the original color image. Then, to remove the remaining undesired reflections or bright skin, the value of every non-black pixel was replaced by the average intensity value of a 17x17 block around it. A binary map was then generated to extract the sclera area. This process allowed for a narrower target area with respect to the size and the location on the image of the sclera in order to accelerate the circle search process for the outer iris boundary detection. 
The outer boundary of the iris was detected using a modified fast Hough circular transform. The modifications considered were specifically established to alleviate the computational burden of the original Hough transform while still seeking a highly accurate segmentation outcome. The same step-length is used for the three iterations of the Hough circular transform, yielding a significantly faster processing speed. The use of this step-length is complemented with an optimization method designed to yield an even higher accuracy in the results.

To overcome various noise effects, a new approach was devised to identify the edge points located on the boundary between eyelid and iris. The identification is based on verifying whether each edge point actually belong to the eyelid and not to the iris region. A linear Hough transform is used recursively in this case to extract the edges of eyelids.

A new method for verification and correction for the outer iris boundary was also devised. The new strategy consisted of (1) verifying and correcting the center position of the outer iris boundary, (2) localizing the target region, (3) performing Hough circular transform with different weights in and out of the target region, and (4) verifying the reliability of the results in terms of the number of edge points which actually belong to the boundary of the iris. As a result, multiple arcs and lines were used to delineate a noncircular iris boundary, and correct the initial inaccurate results of the circular Hough transform.

For the final step, in order to generate a more accurate pupil boundary in the edge map, histogram equalization was used to enhance the contrast between the iris and pupil. 
Consequently, the extracted pupil area is removed from further consideration, allowing for an iris recognition process to focus solely on the delineated iris area.

The video based iris acquisition system as described would release the rigid constraints of the iris verification process so that the iris recognition can be not only accurate but also more reliable and faster. But the iris images obtained from the new acquisition system are usually much lower in resolution than those obtained from the traditional system. And the conventional segmentation methods can not deal with those images very well. This approach was designed towards dealing with various noise effects and the smaller iris size provided by the video acquisition system. However, it is worthy to mention that the proposed approach not only worked well for the NIR face video, it also produced a good performance in color eye images which were taken in the less constrained environment where persons are on the move.

The approach as considered starts from the eye image extraction which is based on the reflections on the eyes generated by the illumination system. And then an adaptive histogram method is used to detect the pupil. The pupil boundary was described by a circle as means to help detect the iris outer boundary, eyelids, and eyelashes. The outer boundary of the iris was detected using the Hough circular transform, some modification was then made on the Hough transform to ease the computational burden and improve accuracy in the detection results. A new eyelids and eyelashes detection method was developed in support of this method in order to overcome the effects of many undesired noise points which hinder the detection process. With our method, the pixels which are on the edge between iris and eyelids or eyelashes will be detected first, and then the Hough line transform is consequently applied. 
Because of off angle, eyelids occultation, small size iris, non-circular iris outer boundaries that would appear more often for the eye images acquired from the less constrained environment, a new method for verification and correction for outer iris boundary was proposed to deal with these unpredictable situations. As such, the method is based on the iris center correction and on performing circle searching algorithm to a select region. Multiple arcs and lines can be used to describe an iris boundary, thus noncircular iris boundary would be described correctly.

Following the segmentation stage, some pioneering experiments on the recognition stage of the less-constrained video iris biometrics have been done. In the video based less-constrained iris recognition, the test or subject iris videos/images and the enrolled iris images are acquired with different acquisition systems. To accelerate the verification or identification process, the test iris images are taken with NIR video while the customers are on the move. The enrolled iris images are on the other hand taken with the conventional acquisition system to ensure the quality of the iris images. In the matching step, the verification/identification result was accomplished by comparing the similarity distance of encoded signature from test images with each of the signature dataset from the enrolled iris images. With the improvements gained, a recognition rate of FAR at 0 and FRR at $17.64 \%$ for 85 tested users is achieved.

For most the intra-class trials which generate the high Hamming distance which eventually lead to a false rejected cases is due mainly to the low quality of the test images. If these low quality images are excluded by an automated quality evaluation process, the FRR would be surely improved. Secondly, for a considerable portion of false accepted cases, the quality of the test image is too low to show enough iris features, meanwhile, 
for a small percent of people, their iris features are relatively less than most of others, which leads to the false acceptance. For this point, the method such as analysis of energy of high spatial frequencies can be conducted to the segmented iris region to lower the false acceptance rate.

Another possible way to improve the results on video based iris recognition is to use different weight to the similarity measures. As can be seen, many of the test images suffer from the occlusion of eye lids and eyelashes, especially for the upper iris region, which refers to the first half of the normalized rectangle iris image. Although, the eye lid detection is performed, it is still difficult to exclude all of the occlusions, especially for the eyelash parts. With a weighted similarity measures, the upper iris would be given by a smaller weight than the lower part of the iris, it would likely improve the matching results.

Through our study, the NIR face video does have the capability to record enough iris information for recognition, but there are also some issues that remain to be addressed within the acquisition system. One of most important is that some of the subjects that walk through the portal miss the NIR flash. In other words, when the flash is on, the subjects are either in front or behind the best focus distance, thus the eye image is not bright enough to retrieve. This issue could be resolved by calculating the speed of the subject and adjust for the best time to turn on the NIR flash.

In retrospect, in light of the encouraging results obtained even with the challenging dynamic environments where subjects are on the move, the new methods for segmentation and iris recognition as proposed show great potential and high practical implications for iris biometrics research and system design. 


\section{BIBLIOGRAPHY}

Adjouadi, M., F. Candocia, et al. (1996). "Exploiting Walsh-based attributes to stereo vision." Ieee Transactions on Signal Processing 44(2): 409-420.

Adjouadi, M., J. Riley, et al. (1995). "An Augmented Computer Vision Approach for Enhanced Image Understanding." Journal of Rehabilitation Research and Development 32(3): 264-279.

Bowyer, K. W., K. Hollingsworth, et al. (2008). "Image understanding for iris biometrics: A survey." Computer Vision and Image Understanding 110(2): 281-307.

Chen, Y., M. Adjouadi, et al. (2010). "A highly accurate and computationally efficient approach for unconstrained iris segmentation." Image and Vision Computing 28(2): 261-269.

Chen, Y., J. Wang, et al. (2008). "A Robust Segmentation Approach to Iris Recognition Based on Video." Proc. Of IEEE-AIPR: 1-8.

Cherri, A. K. and M. A. Karim (1989). "Optical Symbolic Substitution - Edge-Detection Using Prewitt, Sobel, and Roberts Operators." Applied Optics 28(21): 4644-4648.

Daugman, J. (2001). "Iris recognition - The colored part of the eye contains delicate patterns that vary randomly from person to person, offering a powerful means of identification." American Scientist 89(4): 326-333.

Daugman, J. (2001). "Statistical richness of visual phase information: Update on recognizing persons by iris patterns." International Journal of Computer Vision 45(1): 25-38.

Daugman, J. (2003). "The importance of being random: statistical principles of iris recognition." Pattern Recognition 36(2): 279-291.

Daugman, J. (2004). "How iris recognition works." Ieee Transactions on Circuits and Systems for Video Technology 14(1): 21-30.

Daugman, J. (2004). "Recognising persons by their iris patterns." Advances in Biometric Person Authentication, Proceedings 3338: 5-25.

Daugman, J. (2007). "New methods in iris recognition." Ieee Transactions on Systems Man and Cybernetics Part B-Cybernetics 37(5): 1167-1175.

Daugman, J. G. (1993). "High Confidence Visual Recognition of Persons by a Test of Statistical Independence." Ieee Transactions on Pattern Analysis and Machine Intelligence 15(11): 1148-1161. 
Davson, H. (1984). The Eye. Orlando, Academic Press.

de Almeida, P. (2010). "A knowledge-based approach to the iris segmentation problem." Image and Vision Computing 28(2): 238-245.

Fancourt, C., L. Bogoni, et al. (2005). "Iris recognition at a distance." Audio and Video Based Biometric Person Authentication, Proceedings 3546: 1-13.

Flom, L. and A. Safir (1987). "Iris recognition system." U.S. Patent 4,641,349.

Gonzalez, R. C. and R. E. Woods (2008). Digital image processing. Upper Saddle River, N.J., Prentice Hall.

Hollingsworth, K., T. Peters, et al. (2009). "Iris Recognition Using Signal-Level Fusion of Frames From Video." Ieee Transactions on Information Forensics and Security 4(4): $837-848$.

Huang, X., L. Ren, et al. (2009). Image deblurring for less intrusive iris capture. IEEE Conference on Computer Vision and Pattern Recognition. Miami, FL: 1558 1565 .

Jeong, D. S., J. W. Hwang, et al. (2010). "A new iris segmentation method for non-ideal iris images." Image and Vision Computing 28(2): 254-260.

Kanopoulos, N., N. Vasanthavada, et al. (1988). "Design of an Image Edge-Detection Filter Using the Sobel Operator." Ieee Journal of Solid-State Circuits 23(2): 358367.

Kanski, J. J. (2007). Clinical ophthalmology : a systematic approach. Edinburgh ; New York, Butterworth-Heinemann/Elsevier.

Kanski, J. J. and J. Menon (2003). Clinical ophthalmology. Edinburgh ; New York, Butterworth-Heinemann.

Labati, R. D. and F. Scotti (2010). "Noisy iris segmentation with boundary regularization and reflections removal." Image and Vision Computing 28(2): 270-277.

Land, M. F. (1999). "The human eye: structure and function." Nat Med 5(11): 1229.

Leavers, V. F. (1992). Shape detection in computer vision using the Hough transform. London; New York, Springer-Verlag.

Lee, Y., J. P. Phillips, et al. (2008). An Automated Video-Based System for Iris Recognition. Third International Conference on Advances in Biometrics. Alghero, Italy: $1160-1169$. 
Li, P. H., X. M. Liu, et al. (2010). "Robust and accurate iris segmentation in very noisy iris images." Image and Vision Computing 28(2): 246-253.

Ma, L., T. Tan, et al. (2003). "Personal identification based on iris texture analysis." Ieee Transactions on Pattern Analysis and Machine Intelligence 25(12): 1519-1533.

Ma, L., T. N. Tan, et al. (2004). "Efficient iris recognition by characterizing key local variations." Ieee Transactions on Image Processing 13(6): 739-750.

Masek, L. "Recognition of human iris patterns for biometric identification.", Technical Report, 2003

Matey, J. R., R. Broussard, et al. (2010). "Iris image segmentation and sub-optimal images." Image and Vision Computing 28(2): 215-222.

Matey, J. R., O. Naroditsky, et al. (2006). "Iris on the move: Acquisition of images for iris recognition in less constrained environments." Proceedings of the Ieee 94(11): 1936-1947.

Phillips, P. J., K. W. Bowyer, et al. (2007). "Comments on the CASIA version 1.0 iris data set." Ieee Transactions on Pattern Analysis and Machine Intelligence 29(10): 1869-1870.

Phillips, P. J., Scruggs, T., Flynn, P.J., Bowyer, K.W., Beveridge, R., Givens, G., Draper, B., O'Toole, A. (2009). Overview of the multiple biometric grand challenge. Proc. Int. Conf. on Biometrics (ICB 2009)

Proença, H. (2009). On the Feasibility of the Visible Wavelength, At-A-Distance and OnThe-Move Iris Recognition. IEEE Symposium Series on Computational Intelligence in Biometrics: Theory, Algorithms, and Applications. Nashville, Tennessee, U.S.A.: 9-15.

Proenca, H. (2010). "An iris recognition approach through structural pattern analysis methods." Expert Systems 27(1): 6-16.

Proenca, H. (2010). "Iris Recognition: On the Segmentation of Degraded Images Acquired in the Visible Wavelength." Ieee Transactions on Pattern Analysis and Machine Intelligence 32(8): 1502-1516.

Proenca, H. and L. A. Alexandre (2005). "UBIRIS: A noisy iris image database." Image Analysis and Processing - Iciap 2005, Proceedings 3617: 970-977.

Proenca, H. and L. A. Alexandre (2006). "Iris segmentation methodology for noncooperative recognition." Iee Proceedings-Vision Image and Signal Processing 153(2): 199-205. 
Proenca, H. and L. A. Alexandre (2010). "Introduction to the Special Issue on the Segmentation of Visible Wavelength Iris Images Captured At-a-distance and Onthe-move." Image and Vision Computing 28(2): 213-214.

Proenca, H., S. Filipe, et al. (2010). "The UBIRIS.v2: A Database of Visible Wavelength Iris Images Captured On-the-Move and At-a-Distance." Ieee Transactions on Pattern Analysis and Machine Intelligence 32(8): 1529-1535.

Proenca, H., S. Filipe, et al. (2010). "The UBIRIS.v2: a database of visible wavelength iris images captured on-the-move and at-a-distance." IEEE Trans Pattern Anal Mach Intell 32(8): 1529-35.

Ravishankar Rao, A., N.-S. Chang, et al. (1998). Machine vision applications in industrial inspection VI : 27 January 1998, San Jose, California. Bellingham, Wash., USA, SPIE.

Robinett, R. D. (2005). Applied dynamic programming for optimization of dynamical systems. Philadelphia, Society for Industrial and Applied Mathematics.

Ross, A. (2010). "Iris Recognition: The Path Forward." Computer 43(2): 30-35.

Ross, A. and A. K. Jain (2007). "Human recognition using biometrics: an overview." Annales Des Telecommunications-Annals of Telecommunications 62(1-2): 11-35.

Sacco, W. (1987). Dynamic programming : an elegant problem solver. Providence, R.I., Janson Publications.

Sankowski, W., K. Grabowski, et al. (2010). "Reliable algorithm for iris segmentation in eye image." Image and Vision Computing 28(2): 231-237.

Shapiro, L. G. and A. Rosenfeld (1992). Computer vision and image processing. Boston, Academic Press.

Snell, R. S. and M. A. Lemp (1998). Clinical anatomy of the eye. Malden, MA, USA, Blackwell Science.

Tan, T. N., Z. F. He, et al. (2010). "Efficient and robust segmentation of noisy iris images for non-cooperative iris recognition." Image and Vision Computing 28(2): 223230 .

Verdenet, J., J. C. Cardot, et al. (1981). "Scintigraphic Image Contrast-Enhancement Techniques - Global and Local Area Histogram Equalization." European Journal of Nuclear Medicine 6(6): 261-264. 
Wheeler, F. W., A. Perera, et al. (2008). Stand-off Iris Recognition System. 2nd IEEE International Conference on Biometrics: Theory, Applications and Systems. Washington DC, U.S.A.: 1-7.

Wildes, R. P. (1997). "Iris recognition: An emerging biometric technology." Proceedings of the Ieee 85(9): 1348-1363.

Wildes, R. P., J. C. Asmuth, et al. (1996). "A machine-vision system for iris recognition." Machine Vision and Applications 9(1): 1-8.

X. Liu, K. W. B., and P. J. Flynn (2005). Experimental evaluation of iris recognition. Face Recognition Grand Challenge Workshop.

Zhou, Z., Y. Z. Du, et al. (2009). "Transforming Traditional Iris Recognition Systems to Work in Nonideal Situations." Ieee Transactions on Industrial Electronics 56(8): 3203-3213. 
VITA

YU CHEN

Education

$\begin{array}{ll}1999-2003 & \begin{array}{l}\text { B.E., Computer Science } \\ \text { Xuzhou Normal University } \\ \text { Xuzhou, Jiangsu, PR. China }\end{array} \\ & \text { M.E., Electrical Engineering } \\ & \text { Florida International University } \\ & \text { Miami, FL } \\ & \\ & \text { Doctoral Candidate in Electrical Engineering } \\ & \text { Florida International University } \\ & \text { Miami, FL } \\ & \text { Research Assistant } \\ & \text { Center for Advance Technology and Education } \\ & \text { (CATE) } \\ & \text { Florida International University } \\ & \text { Miami, FL }\end{array}$

Publications

JOURNAL ARTICLE

1. Y. Chen, M. Adjouadi, C. Han, J. Wang, A. Barreto, N. Rishe, and J. Andrian, "A Highly Accurate and Computationally Efficient Approach for Unconstrained Iris Segmentation", Image and Vision Computing, Volume 28, Issue 2, February 2010, Pages 261-269.

\section{ARTICLE APPEARING AS BOOK CHAPTER}

2. Y. Chen and M. Adjouadi, Chapter Title: Iris; in Encyclopedia of Cryptography and Security (2nd ed.), Springer, 2010.

\section{REFEREED ARTICLES in CONFERENCE PROCEEDINGS}

3. Y. Chen, J. Wang, and M. Adjouadi, "A Robust Segmentation Approach to Iris Recognition Based on Video", the 37th IEEE Applied Imagery Pattern Recognition (AIPR08) annual workshops, Washington DC, Oct. 15-17, 2008, pp:1 - 8, DOI: 10.1109/AIPR.2008.4906441. 
4. Y. Chen, M. Adjouadi, C. Han and A. Barreto "A new unconstrained iris image analysis and segmentation method in biometrics", IEEE International Symposium on Biomedical Imaging, ISBI, Boston, MA, U.S.A., Jun 28-Jul 1, 2009, pp.13-16, DOI: 10.1109. (Peer-reviewed)

5. Y. Chen and M. Adjouadi, "A New Noise Tolerant Segmentation Approach to Non-ideal Iris Image with Optimized Computational Speed". The 2009 International Conference on Image Processing, Computer Vision, \& Pattern Recognition, IPCV 2009, July 13-16, 2009, Las Vegas Nevada, USA, 2 Volumes. CSREA Press 2009, ISBN 1-60132-119-8, pp. 547-553. (Peer-reviewed, Acceptance rate: $25 \%$ )

6. Y. Chen, M. Adjouadi, A. Barreto, N. Rishe, and J. Andrian, “A Computational Efficient Iris Extraction Approach in Unconstrained Environments", IEEE International Conference on Biometrics: Theory, Applications and System (BTAS 09), Washington DC, Sep. 28-30, 2009, pp. 1-7. (Peer-reviewed, Acceptance rate: 20\%; Best student paper nominee: $7 / 70+$ ).

7. J. Wang, A. Barreto, L. Wang, Y. Chen, N. Rishe, J. Andrian, M. Adjouadi, "Multilinear Principal Component Analysis for Face Recognition with Fewer Features", Neurocomputing, No. NEUCOM-D-09-00277R1, Accepted, to appear in 2010 .

8. J. Wang, Y. Chen, and M. Adjouadi, "A Comparative Study of the Multi-linear PCA for Face Recognition", the 37th IEEE Applied Imagery Pattern Recognition (AIPR08) annual workshops, Washington DC, Oct. 15-17, 2008, pp:1 - 8, DOI: 10.1109/AIPR.2008.4906476.

9. Wu Sheng and Yu Chen "A Study of J2ME based Mobile Ticketing Systems", Fujian Computer (2003), 10:14-15, ISSN: 1673-2782, CN: 35-115/TP. (in Chinese) 